\title{
A cholinergic medial septum input to medial habenula mediates generalization formation and extinction of visual aversion
}

\section{Rong-Hao Mu}

China Pharmaceutical University

\section{Xiao-Meng Han}

China Pharmaceutical University

\section{Hao Wang}

China Pharmaceutical University

\section{Su-Su Tang}

China Pharmaceutical University

\section{Yan Long}

China Pharmaceutical University

\section{Dan-Hua Yuan}

China Pharmaceutical University

Hao Hong ( $\nabla$ honghao@cpu.edu.cn )

China Pharmaceutical University

\section{Article}

Keywords: Affective Disorders, Aversion, Cholinergic Circuits, Glutamatergic Neurons

Posted Date: January 8th, 2021

DOI: https://doi.org/10.21203/rs.3.rs-127062/v1

License: (c) (1) This work is licensed under a Creative Commons Attribution 4.0 International License. Read Full License 
A cholinergic medial septum input to medial habenula mediates generalization formation and extinction of visual aversion

3 Ronghao Mu, Xiaomeng Han, Hao Wang, Susu Tang, Yan Long, Danhua Yuan, Hao Hong*

Department of Pharmacology, Key Laboratory of Neuropsychiatric Diseases, China Pharmaceutical University, Nanjing 211198, China

*Address correspondence to:

Hao Hong, Prof.

Department of Pharmacology, China Pharmaceutical University, Nanjing 211198, China

E-mail: honghao@cpu.edu.cn; Tel: +86-25-86185328 


\section{$24 \quad$ Abstract}

Generalization formation and extinction of aversion are associated with affective disorders, but little is known about underlying mechanisms. Here, we established a novel procedure for induction of visual aversion by dynamic stripe images on digital screens in mice. We found that decreased activity of medial septum (MS) cholinergic neurons led to generalization aversion loss, but didn't affect its extinction. We identified a new projection from MS cholinergic neurons to medial habenula (MHb), and found that inhibiting $\mathrm{MS} \rightarrow \mathrm{MHb}$ cholinergic circuit disrupted generalization formation, while activating this circuit damaged extinction. The further studies showed that blockade of M1 mAChRs rather than $\alpha 4 \beta 2$ and $\alpha 7 \mathrm{nAChRs}$ on downstream glutamatergic neurons that corelease glutamate and acetylcholine blunted generalization enhancement and extinction deficit caused by activation of $\mathrm{MS} \rightarrow \mathrm{MHb}$ circuits. These findings reveal that $\mathrm{MS} \rightarrow \mathrm{MHb}$ cholinergic circuit modulates generalization formation and extinction of aversion, providing new insights on affective disorders such as PTSD and anxiety disorders. 


\section{Introduction}

When we are exposed to an aversive situation, which may be life threatening, fear or avoidance is the normal adaptive response, indispensable to prevent harmful situations ${ }^{1}$. Acquisition and storage of aversive memories is one of the basic principles of central nervous systems $(\mathrm{CNS})^{2}$. In the absence of reinforcement, the resulting behavioural response will gradually diminish to be finally extinct. Extinction of aversive memories is thought to be an active mnemonic process ${ }^{3}$. Generalization is often described as an inability to discriminate different stimuli ${ }^{4}$. Overgeneralization or extinction deficit may lead to inadequate physiological and behavioral responses that affect daily life ${ }^{5}$. Facilitating the extinction of aversive memory and/or attenuating its generalization could have therapeutic implication in the treatment of some diseases such as post-traumatic stress disorder (PTSD), anxiety disorders, and etc $^{6,7}$.

Great progress has been made in studies using a form of aversive associative learning termed fear conditioning. It has been demonstrated that fear learning is engaged through multiple, parallel aversive signaling pathways to hippocampus and amygdala ${ }^{8,9,10}$. Some studies indicate that aversive taste memories are established and sustained by parabrachial nucleus calcitonin-gene-related peptide neurons ${ }^{11}$, and the memory of conditioned taste aversion may be regulated by PI3K in the insular cortex $^{12}$. However, there are few reports on visual aversion memory. It is well known that cholinergic neurons are involved in various memories ${ }^{13}, 14,15,16$. Most of the cholinergic projections to cerebral cortex, hippocampus, and amygdala with established roles in memory, fear and anxiety, originate from the basal forebrain, a collection of heterogeneous structures that includes the medial septum (MS) ${ }^{17,18}$. Previous studies have shown that cholinergic neurons in the MS maintain anxiety-like behaviors induced by chronic inflammatory pain $^{19}$ and inhibiting these neurons with DREADD (designer receptors exclusively activated by designer drugs) alleviates anxiety-like behaviors in mice ${ }^{20}$. 
Moreover, disruption of the MS and diagonal bands of Broca cholinergic projections to the ventral hippocampus damages auditory fear memory ${ }^{21}$. The medial habenula (MHb) is a brain center known to regulate negative states ${ }^{22,23,24}$. Previous studies have shown that mu opioid receptors in the $\mathrm{MHb}$ contribute to naloxone aversion ${ }^{25}$, and endocannabinoid control of $\mathrm{MHb}$ cholinergic neurotransmission exerts a critical role in the expression of aversive memories ${ }^{26}$. We are curious whether or how the neurons in MS and MHb modulate visual aversion memory. To address these questions, we established a novel procedure for induction of visual aversion by a dynamic stripe image on digital screens in mice and used apoptotic, optogenetic, chemogenetic and whole-cell patch-recording approaches to investigate the roles of neurons in these two regions in generalization formation and extinction of visual aversion.

\section{Results}

\section{MS provides strong cholinergic projections to the MHb}

MS, an important part of basal forebrain cholinergic system, is the major source of cholinergic (as well as non-cholinergic) projections to cingulate cortex, entorhinal cortex and hippocampus ${ }^{27}$. To track MS novel cholinergic projections, we stereotaxically injected AAV-chat-cre-EGFP vector into the MS (Fig. 1a, b). Staining with anti-choline acetyltransferase (ChAT) revealed that $96.3 \pm 0.7 \%$ of EGFP-labeled cells were $\mathrm{ChAT}^{+}$neurons, and $92.5 \pm 1.2 \%$ of ChAT-stained neurons were EGFP-labeled cells (Fig. 1c, d). The cholinergic neurons were also identified by their response to intracellular current injection during whole-cell patch-clamp recordings (Fig. 1e, f). Injection of a negative current pulse produced an initial hyperpolarization followed by a cation conductance $I_{h}$-dependent sag in the membrane potential while depolarizing current induced regular spiking and resulted in a long-lasting afterhyperpolarization after cessation of current injection (Fig. 1f). Additionally, in the absence of applied current, spontaneous spiking was observed in this particular neuron. Using virus tracing, dense 
networks of MS cholinergic projections were observed in the whole MHb (Fig. 1g). These data reveal that MS provides strong cholinergic projections to the MHb in addition to the projections reported previously.

\section{MS cholinergic neuron supports rapid generalization formation and extinction of visual aversion}

Visual aversive images are often used in aversive learning which modulates perceptual thresholds on overgeneralization $^{28}$. To determine whether an image was aversive in mice, we firstly employed realtime place preference (RTPP) caused by vision. In brief, mice were allowed to freely explore a twochamber apparatus in which each chamber contained four digital screens that displayed one of three images: a blank image, a static stripe image, or the same stripe image that was moving (named as dynamic stripe image), respectively. We found that mice spent significantly less time in the chamber displaying the dynamic stripe image relative to those displaying either the static stripe or blank images, suggesting dynamic stripe images are aversive images. The time exploring the chamber with static stripe images was the same as one with the blank, showing the static images are neutral images (Fig. 2a-c and Supplementary Fig. 1). We also submitted those mice to open field test (OFT) and elevated plus-maze (EPM) (Supplementary Fig. 2a). The data showed that mice exposed to the dynamic image exhibited significant reductions in exploration time of center part in OFT and open arms in EPM while no obvious change in freezing time (Supplementary Fig. 2b-e), suggesting that visual stimulations of dynamic images lead to generalized anxiety in mice.

Similar with fear, aversion is involved in generalization and extinction ${ }^{29,30,31}$. To explore generalization formation and extinction processes in visual aversion, we designed a task using the highly similar dynamic and static stripe images referring to previous studies ${ }^{32,33,34,35}$ (Fig. 2d). In brief, after two habituation phases of the apparatus, mice were aversively stimulated for 10 min using the dynamic stripe image, and after $1 \mathrm{~h}$, aversion generalization formation was examined using the 
corresponding static stripe image in one chamber of the apparatus. After a $4 \mathrm{~h}$-delay, we performed an aversion generalization extinction test with the same approach. During habituation phase 1 and 2, mice did not exhibit any differences in the time spent exploring chambers, suggesting that there was no side preference (phase 1) and the static image served as a neutral stimulus (phase 2). After exposure to dynamic images in training phase, mice spent only $34.8 \pm 1.5 \%$ of the whole time in the chamber with static stripe images in test phase 1 (Fig. 2e), suggesting that the static image, which was a visual neutral image before, now elicited a significant aversion avoidance response in mice, indicating an aversion generalization formation. After a $4 \mathrm{~h}$-delay, $48.7 \pm 1.0 \%$ of the whole time spent by mice in the stripe image chamber showed that mice no longer exhibited aversion for the static image during test phase 2 (Fig. 2f), revealing a generalization extinction of visual aversion. However, if mice were tested without dynamic image stimulations in training phase, the static image could not cause generalized aversion behaviors during test phases (Fig. 2g-i). Moreover, we also used a new two-chamber apparatus with a white floor in test phases, and found this modified context did not affect aversion generalization processes (Supplementary Fig. 3). Altogether, these data reveal that there are rapid generalization formation and extinction of visual aversion for the dynamic stripe image.

Using c-Fos as a proxy for neuronal activity, we found much higher c-Fos expression in MS cholinergic neurons in aversion generalization formation phase (test phase 1) than both in the extinction phase (test phase 2) and the test phases without aversive stimulations of dynamic images (Fig. 2j, k). We also measured the $\mathrm{Ca}^{2+}$ signals of MS cholinergic neurons with fiber photometry in freely moving mice after stereotaxic virus injection of AAV-chat-cre and the cre-dependent AAVEf1 $\alpha$-DIO-GCaMP6m into the MS (Fig. 21-n). Real-time recording of GCaMP fluorescence signals of MS cholinergic neurons revealed a rapid increase in $\mathrm{Ca}^{2+}$ signal when the moving mouse first entered the chamber containing static stripe images during the generalization formation phase (test phase 1; 
Fig. 3o, left), and $\mathrm{Ca}^{2+}$ signals did not significantly change upon entry into the chamber containing static images during the generalization extinction phase (test phase 2; Fig. 2o, right). In addition, the mice unexposed to dynamic images in training phase had a mild increase in fluorescence signal in test phase 1 (Fig. 2p, left) while no change in test phase 2 (Fig. 2p, right). Altogether, these data suggest that MS cholinergic neurons might be involved in generalization formation and extinction of visual aversion.

\section{MS cholinergic neuron activity is necessary for generalization formation and extinction of visual}

\section{aversion}

To test whether MS is necessary for generalization formation and extinction, we manipulated MS cholinergic neurons with apoptosis, chemogenetic, and optogenetic approaches. Firstly, after stereotaxic virus injection of AAV-chat-cre-EGFP into the MS, we promoted MS apoptosis with a credependent AAV-CAG-DIO-taCaspase3 (Fig. 3a). The apoptotic approach resulted in a $72 \%$ reduction of EGFP-labeled cholinergic neurons in the MS in taCaspase3-expressing mice (Fig. 3b, c). In aversion generalization task, taCaspase3-expressing mice had no aversion avoidance response to the stripe image chamber in the generalization formation phase compared with the control mice (Fig. 3d, e), suggesting that the taCaspase3-expressing mice failed to form a generalized aversion to the static stripe image. To explore whether chemogenetic and optogenetic inhibitions of MS cholinergic neurons disrupt formation of aversion generalization, cre-dependent AAV-Ef1 $\alpha$-DIO-hM4D(Gi)-mCherry (Supplementary Fig. 4a) or AAV-Ef1 $\alpha$-DIO-NpHR-mCherry (Fig. 3g) was injected into the MS, respectively. $92.6 \pm 1.3 \%$ of EGFP-labeled MS cholinergic neurons expressed hM4D(Gi)-mCherry for chemogenetic inhibition (Supplementary Fig. 5a, b), while $89.8 \pm 1.6 \%$ of EGFP-labeled MS cholinergic neurons expressed NpHR-mCherry for optogenetic inhibition (Supplementary Fig. 5c, d). Acute MS slices containing NpHR-mCherry-expressing were prepared, and brief yellow-light (589 
$\mathrm{nm}$ ) photostimulation elicited obvious decreases in spontaneous firing on the neurons. (Fig. $3 \mathrm{~h}$ ). As observed with the apoptotic approach, clozapine N-oxide (CNO)-induced chemogenetic inhibition or yellow-light-induced optogenetic inhibition during test phase 1 (Fig. 3f) led to similar exploration in two chambers (Fig. 3i, j and Supplementary Fig. 4b, c). These data revealed that apoptosis, chemogenetic and optogenetic inhibitions of MS cholinergic neurons disrupted generalization formation of visual aversion.

To activate MS cholinergic neurons, cre-dependent AAV-Ef1 $\alpha$-DIO-hM3D(Gq)-mCherry (Fig. 3k) or AAV-Ef1 $\alpha$-DIO-ChR2-mCherry (Supplementary Fig. 4e) was injected into the MS, respectively. We found $88.2 \pm 1.6 \%$ of EGFP-labeled MS cholinergic neurons expressed $\mathrm{hM} 3 \mathrm{D}(\mathrm{Gq})$-mCherry (Supplementary Fig. 5e, f), $91.5 \pm 2.1 \%$ of EGFP-labeled MS cholinergic neurons expressed ChR2mCherry (Supplementary Fig. 5g, h), and $95.6 \pm 1.1 \%$ of EGFP-labeled MS cholinergic neurons expressed mCherry alone (Supplementary Fig. 5i, j). Moreover, increased spontaneous firing of $\mathrm{hM} 3 \mathrm{D}(\mathrm{Gq})$-mCherry-expressing neurons after CNO application to MS slices was also observed (Fig. 31). In aversion generalization task, mice were intraperitoneally injected $\mathrm{CNO}(2.5 \mathrm{mg} / \mathrm{kg})$ to activate MS cholinergic neurons after generalization formation phase. The data of test phase 2 showed that the chemogenetic activation of MS cholinergic neurons resulted in a significant aversion response to the stripe image chamber in $\mathrm{hM} 3 \mathrm{D}(\mathrm{Gq})$-expressing mice (Fig. 3m, n). Similar results were obtained in optogenetic manipulation (Supplementary Fig. 4d, f, g). These data revealed that continuous activations of MS cholinergic neurons disrupted generalization extinction of visual aversion.

In addition, to further evaluate whether apoptosis or chemogenetic inhibition/activation of MS cholinergic neurons lead to gross behavioural abnormality in mice, we conducted OFT, EPM-test, grip strength test (GST), and novel object recognition test (NORT). The results showed that manipulations of MS cholinergic neurons did not change gross behaviors (Supplementary Fig. 6). 


\section{$\mathrm{MS} \rightarrow \mathrm{MHb}$ cholinergic circuit drives generalization formation and extinction of visual aversion}

To dissect the role of the MS $\rightarrow$ MHb cholinergic circuit in visual aversion generalization, we specially inhibited or activated this circuit with chemogenetic or optogenetic manipulations. For inhibition of the $\mathrm{MS} \rightarrow \mathrm{MHb}$ circuit, AAV2/9-chat-cre-EGFP vector was injected into the MS, whereas credependent, monosynaptic retrograde tracing virus AAV2/Retro-Ef1 $\alpha$-DIO-hM4D(Gi)-mCherry or AAV2/Retro-Ef1 $\alpha$-DIO-NpHR-mCherry was bilaterally injected into the MHb, respectively (Fig. 4a, b and Supplementary Fig. 7a, b). After three weeks, aversion generalization tasks were submitted to the treated or control mice. Both wild-type and hM4D(Gi)-expressing mice were injected with $\mathrm{CNO}$ $(2.5 \mathrm{mg} / \mathrm{kg})$ intraperitoneally $30 \mathrm{~min}$ before generalization formation test. Additionally, the cannulas were bilaterally implanted in the MHb of wild-type and NpHR-expressing mice one week before tasks, and yellow-light photoinhibition was delivered during generalization formation test. The data showed that the $\mathrm{hM} 4 \mathrm{D}(\mathrm{Gi})$ - or NpHR-expressing mice had no aversion avoidance response to the stripe image chamber in the aversion generalization formation phase (Fig. 4c, d and Supplementary Fig. 7c, d), indicating that inhibition of the $\mathrm{MS} \rightarrow \mathrm{MHb}$ cholinergic circuit disrupted generalization formation of visual aversion.

For activation of MS $\rightarrow \mathrm{MHb}$ circuits, AAV2/9-chat-cre-EGFP vector was injected into the MS, whereas cre-dependent, monosynaptic retrograde tracing virus AAV2/Retro-Ef1 $\alpha$-DIO-hM3D(Gq)mCherry (Supplementary Fig. 7e) or AAV2/Retro-Ef1 $\alpha$-DIO-ChR2-mCherry (Fig. 4f) was bilaterally injected into the MHb, respectively. After 3-week virus expressions (Fig. 4g and Supplementary Fig. 7f), CNO intraperitoneal injection after generalization formation phase or blue-light photostimulation during generalization and extinction phases was performed to activate this circuit (Fig. 4e). We found that continuous activations of the $\mathrm{MS} \rightarrow \mathrm{MHb}$ cholinergic circuit disrupted aversion generalization extinction (Fig. 4h, i and Supplementary Fig. 7g, h). Moreover, we also showed that chemogenetic 
manipulations of the MS $\rightarrow \mathrm{MHb}$ circuit showed no significant alterations in behaviors in Morris water maze (MWM) and Y-maze tests (Supplementary Fig. 8), suggesting the MS $\rightarrow$ MHb cholinergic circuit mainly regulates visual aversion generalization rather than spatial working memory. Figure 1 have showed that hippocampus $\mathrm{CA} 3$, as a key memory center in brain, received cholinergic projections from the MS. To explore whether the MS $\rightarrow \mathrm{CA} 3$ cholinergic circuit is involved in visual aversion generalization, we inhibited this circuit with optogenetic approach. The results showed that inhibition of $\mathrm{MS} \rightarrow \mathrm{CA} 3$ projections did not disrupt movement, object recognition and visual aversion generalization in mice (Supplementary Fig. 9). Altogether, the data reveal that MS $\rightarrow$ MHb cholinergic circuit drives generalization formation and extinction of visual aversion.

\section{M1 mAChRs mediate regulatory effects of the MS $\rightarrow$ MHb circuit on visual aversion}

\section{generalization}

The most commonly expressed mAChRs in the CNS are M1, M2 and M4 receptors ${ }^{36}$ and the most common nAChR subtypes in the brain are $\alpha 4 \beta 2$ and $\alpha 7$ receptors $^{37}$. Because MHb contains M1, $\alpha 4$, $\alpha 7$ and $\beta 2$ polypeptides (Supplementary Fig. 10a), it is likely that M1, $\alpha 4 \beta 2$ and $\alpha 7$ AChRs mediate regulatory effects of the MS $\rightarrow \mathrm{MHb}$ circuit on visual aversion generalization. We bilaterally injected $\alpha 4 \beta 2$ and $\alpha 7$ AChRs antagonist benzethonium chloride $(2 \mu \mathrm{g} / 0.1 \mu 1 /$ side $)$ and M1 mAChRs antagonist pirenzepine dihydrochloride $(1.5 \mu \mathrm{g} / 0.1 \mu \mathrm{l} / \mathrm{side})$ into the $\mathrm{MHb}$, respectively. The data showed that inhibition of $\alpha 4 \beta 2$ and $\alpha 7 \mathrm{nAChRs}$ in the $\mathrm{MHb}$ did not influence aversion generalization formation and extinction, whereas inhibition of M1 mAChRs disrupted generalization formation (Supplementary Fig. 10b-d), suggesting M1 mAChRs rather than $\alpha 4 \beta 2$ and $\alpha 7 \mathrm{nAChRs}$ mediate regulatory effects of $\mathrm{MHb}$ neurons on generalization process of visual aversion.

Because MHb acetylcholine signaling transmission is instrumental for the MS $\rightarrow \mathrm{MHb}$ circuit. To further confirm role of acetylcholine signals, after virus AAV2/9-chat-cre-EGFP and cre-dependent 
AAV2/Retro-Ef1 $\alpha$-DIO-hM3D(Gq)-mCherry expressions, we combined targeted expression of hM3Dq in the circuit with direct bilateral microinjections of $\mathrm{CNO}(3.0 \mu \mathrm{M} / 0.1 \mu \mathrm{l} / \mathrm{side})$ or a mixture of pirenzepine $(1.5 \mu \mathrm{g} / 0.1 \mu \mathrm{l} / \mathrm{side})$ into the MHb (Fig. 5a, b). The results showed that chemogenetic activation of MS $\rightarrow \mathrm{MHb}$ cholinergic circuits significantly enhanced aversion generalization formation, whereas blockade of MHb M1 mAChRs attenuated this effect (Fig. 5c, d). These data show that M1 mAChRs mediate regulatory effects of the $\mathrm{MS} \rightarrow \mathrm{MHb}$ cholinergic circuit on generalization of visual aversion.

\section{MHb glutamatergic/cholinergic neurons participate in generalization formation and extinction}

\section{of visual aversion}

$\mathrm{MHb}$ is mainly composed of glutamatergic neurons which can project to the interpeduncular nucleus (IPN) and other regions ${ }^{38}$. To explore whether MHb glutamatergic neurons participate in visual aversion generalization, we injected AAV2/9-CaMKII $\alpha$-cre and an acetylcholine optical sensor virus AAV2/9-Ef1 $\alpha$-DIO-ACh2.0 into the MHb ${ }^{39}$. After three weeks, acetylcholine fluorescence signals in MHb glutamatergic neurons were recorded using a fiber photometry in freely moving mice submitted to aversion generalization task (Fig. 5e, f). The results showed an acute increase in acetylcholine signals upon entering the static stripe image chamber during generalization formation phase of visual aversion, but acetylcholine signals did not significantly change during the generalization extinction phase (Fig. 5g), suggesting that MHb glutamatergic neurons might be involved in visual aversion generalization and receive cholinergic inputs from the MS. Next, we chemogenetically activated $\mathrm{MS} \rightarrow \mathrm{MHb}$ cholinergic circuits and inhibited $\mathrm{MHb}$ glutamatergic neurons together by the bilateral injections of AAV2/Retro-Ef1 $\alpha$-DIO-hM3D(Gq) and AAV2/9-CaMKII $\alpha$-hM4D(Gi) into the MHb after the expression of AAV2/9-chat-cre in the MS. CNO $(3.0 \mu \mathrm{M} / 0.1 \mu 1 /$ side $)$ was bilaterally injected into the MHb 30 min prior to the behavioral test (Fig. 6a, b). The results showed that inhibition of 
$\mathrm{MHb}$ glutamatergic neurons blunted enhancement of aversion generalization formation caused by chemogenetic activation of MS $\rightarrow$ MHb cholinergic circuits (Fig. 6c, d).

Previous study has reported that some MHb neurons could corelease glutamate and acetylcholine (name as glutamatergic/cholinergic neurons $)^{40}$. After staining MHb slices with anti-CaMKII $\alpha$ and anti-ChAT, we showed that $38.4 \pm 2.7 \%$ of $\mathrm{CaMKIII}^{+}$neurons co-expressed ChAT, and $97.8 \pm 0.8 \%$ of $\mathrm{ChAT}^{+}$neurons co-expressed CaMKII $\alpha$ (Fig. 6e). To confirm the roles of $\mathrm{MHb}$ glutamatergic/cholinergic neurons in visual aversion generalization, we chemogenetically inhibited $\mathrm{MS} \rightarrow \mathrm{MHb}$ cholinergic circuits and activated $\mathrm{MHb} \mathrm{CaMKII} \alpha^{+} \mathrm{ChAT}^{+}$neurons by bilateral injections of AAV2/9-chat-cre and AAV2/9-Ef1 $\alpha$-DIO-hM3D(Gq) into the MHb after expressions of AAV2/9chat-cre and AAV2/9-Ef1 $\alpha$-DIO-hM4D(Gi) in the MS. CNO $(3.0 \mu \mathrm{M} / 0.1 \mu \mathrm{l} / \mathrm{side})$ was bilaterally injected into the MHb 30min prior to the behavioral test (Fig. 6f, g). The results showed that activation of $\mathrm{MHb} \mathrm{CaMKII} \alpha^{+} \mathrm{ChAT}^{+}$neurons protected against aversion generalization deficit caused by chemogenetic inhibition of $\mathrm{MS} \rightarrow \mathrm{MHb}$ cholinergic circuits (Fig. 6h, i). Moreover, there was a tendency to disrupt generalization extinction with this activation approach $(P=0.059)$. These data show that MHb glutamatergic/cholinergic neurons participate in generalization of visual aversion regulated by MS $\rightarrow \mathrm{MHb}$ cholinergic circuits.

To explore underlying mechanisms of MS $\rightarrow \mathrm{MHb}$ cholinergic circuits mediating visual aversion generalization, after labeling MHb neurons with AAV2/9-CaMKIIa-mCherry and AAV2/9-chatEGFP vectors, we optogenetically activated the MS $\rightarrow$ MHb circuit by injections of AAV2/9-chat-cre and AAV2/9-Ef1 $\alpha$-DIO-ChR2-mCherry into the MS, and tested the excitatory postsynaptic currents (EPSCs) of MHb neurons with whole-cell recording (Fig. 7a, b). The results showed that optogenetic activation of MS $\rightarrow \mathrm{MHb}$ cholinergic circuits significantly enhanced the frequency and amplitude of EPSCs of MHb CaMKII $\alpha^{+} \mathrm{ChAT}^{+}$neurons and didn't do in the present of M1 mAChRs antagonist 
pirenzepine (Fig. 7c-e). However, the EPSCs of MHb glutamatergic neurons labelled with CaMKII $\alpha$ alone (MHb CaMKII $\alpha^{+} \mathrm{ChAT}^{-}$neurons) did not significantly change under activation of $\mathrm{MS} \rightarrow \mathrm{MHb}$ circuits (Fig. 7c-e). Taken together, MS $\rightarrow$ MHb cholinergic projections modulate generalization formation and extinction of visual aversion via M1 mAChRs on downstream glutamatergic/cholinergic neurons.

\section{Discussion}

Our studies showed for the first time that MS cholinergic neurons were activated in visual aversion generalization formation and silenced in its subsequent extinction as indicated by immunofluorescence assay of c-Fos and fiber photometry of $\mathrm{Ca}^{2+}$ signal, and apoptosis or inhibitions of MS cholinergic neurons damaged generalization formation, whereas the activations of the MS disrupted its extinction process. We also found that MS cholinergic neurons could project to the MHb, which indicates existence of the MS $\rightarrow \mathrm{MHb}$ cholinergic circuit, and this circuit bidirectionally regulated generalization and extinction in visual aversion, and blockade of M1 mAChRs rather than $\alpha 4 \beta 2$ and $\alpha 7 \mathrm{nAChRs}$ on $\mathrm{MHb}$ glutamatergic/cholinergic neurons weakened aversion generalization enhancement and extinction deficiency caused by activation of MS $\rightarrow \mathrm{MHb}$ cholinergic circuits. Collectively, our results provide strong evidence that the MS $\rightarrow \mathrm{MHb}$ cholinergic circuit is both sufficient and necessary to rapidly mediate generalization formation and extinction in visual aversion (Fig. 8).

Formation and extinction are two important phases in generalization, and although generalization is a critical function of the brain that supports survival and avoids danger, overgeneralization and extinction dysfunction of aversion contributes to neurological diseases ${ }^{41,42,43,44}$. To explore generalization processes, typically behavioral tasks based on contextual fear conditioning with electric shock and auditory stimulus are always performed in animals ${ }^{45}, 46$. However, the visual and auditory systems frequently work together to facilitate the identification and localization of objects and events 
in the external world, and for stimulus location, visual information is normally more accurate and reliable and provides a reference for calibrating the perception of auditory space ${ }^{47}$. A possible key role of vision in the development of schizophrenia was reported in previous studies ${ }^{48}$. Additionally, vision is the primary sense humans use to evaluate and respond to threats, and human with high anxiety showed increased visual scanning in response to threats as compared to healthy controls ${ }^{49}$. Young- and middle-age adults with worse visual function are at increased odds of having anxiety disorders ${ }^{50}$. Giving up contextual fear conditioning, we have established a novel procedure for induction of visual aversion by a dynamic stripe image (aversive image) on digital screens in mice, and procedures of aversion generalization formation and extinction are based on highly similarity between the dynamic stripe image and its static stripe image (neutral image).

The cholinergic neurons constitute about $5 \%$ of the MS population, while the majority of MS neurons are GABAergic and glutamatergic ${ }^{51}$. In the present study, we have shown for the first time that MS cholinergic neurons are both sufficient and necessary to rapidly mediate visual aversion generalization. MS projects to the hippocampus by septo-hippocampal pathways are always reported. Previous studies have shown MS GABAergic projections to the dentate gyrus control adult hippocampal neurogenesis ${ }^{52}$, and its glutamatergic projections drive hippocampal theta rhythms ${ }^{53}$. Here, after virus tracking MS cholinergic projections, we focus on the MS $\rightarrow \mathrm{MHb}$ circuit and show that $\mathrm{MS} \rightarrow \mathrm{MHb}$ cholinergic circuits rather than $\mathrm{MS} \rightarrow \mathrm{CA} 3$ projections in the hippocampus bidirectionally regulate visual aversion generalization formation and extinction. The $\mathrm{MHb}$, as an epithalamic area controlling aversive physiological states, also regulates spatial learning and memory ${ }^{54}$ 55. With chemogenetically activating and inhibiting approaches, we have shown that $\mathrm{MS} \rightarrow \mathrm{MHb}$ cholinergic circuits mainly mediate aversion generalization rather than spatial working memory.

M1 mAChRs and $\alpha 4 \beta 2$ and $\alpha 7 \mathrm{nAChRs}$ are commonly expressed in the brain and play key roles 
in attention and memory ${ }^{15}$. M1 mAChRs signal mediated by $\mathrm{G}_{\mathrm{q} / 11}$ while $\alpha 4 \beta 2$ and $\alpha 7 \mathrm{nAChRs}$ signal by ion channels. The distributions of $\mathrm{M} 1, \alpha 4, \alpha 7$ and $\beta 2$ polypeptides in the MHb are shown by in situ hybridization from Allen Institute for Brain Science. Using M1 mAChRs antagonist ${ }^{56,57}$ and $\alpha 4 \beta 2$ and $\alpha 7$ nAChRs antagonist ${ }^{58}$, we have shown M1 mAChRs rather than $\alpha 4 \beta 2$ and $\alpha 7 \mathrm{nAChRs}$ on downstream neurons of $\mathrm{MS} \rightarrow \mathrm{MHb}$ cholinergic circuits mediate visual aversion generalization. Previous studies have reported that $\mathrm{MHb}$ glutamatergic/cholinergic neurons control expressions of aversive and fear memory ${ }^{26,}{ }^{59}$. Staining $\mathrm{MHb}$ neurons with $\mathrm{ChAT}$ and CaMKII $\alpha$ by immunohistochemistry suggested that nearly all cholinergic neurons could corelease glutamate but a part of $\mathrm{MHb}$ glutamatergic neurons could corelease acetylcholine. Activations of $\mathrm{MHb}$ glutamatergic/cholinergic neurons weakened aversion generalization deficiency caused by inhibition of $\mathrm{MS} \rightarrow \mathrm{MHb}$ cholinergic circuits, and M1 AChRs antagonist suppressed significant increase of frequency and amplitude of EPSCs of the glutamatergic/cholinergic neurons under acitivating $\mathrm{MS} \rightarrow \mathrm{MHb}$ cholinergic circuits. Based on these data, our studies have shown that MHb glutamatergic/cholinergic neurons mediate regulatory effects of MS $\rightarrow \mathrm{MHb}$ cholinergic circuits on visual aversion generalization via m1 MAChRs. Because MHb glutamatergic/cholinergic neurons mainly provide projections to the IPN and the $\mathrm{MHb} \rightarrow$ IPN inputs play key roles in anxiety ${ }^{60,61}$, we speculate that IPN might be a targeted region mediating generalization formation and extinction of visual aversion following the $\mathrm{MS} \rightarrow \mathrm{MHb}$ cholinergic circuit.

Overall, taking advantage of virus tracking, optogenetic and chemogenetic approaches as well as novel behavioral paradigm associated with visual aversion, we have identified $\mathrm{MS} \rightarrow \mathrm{MHb}$ cholinergic circuit, and firstly provide highly consistent behavioral evidences for this circuit functions in generalization formation and extinction of visual aversion. Therefore, these results strongly suggest $\mathrm{MS} \rightarrow \mathrm{MHb}$ cholinergic circuit as new targets for therapeutically protecting against affective disorders 
such as PTSD, anxiety disorders and etc.

\section{Methods}

Animals. Male SPF grade C57BL/6 mice (8-10 weeks old) provided by the Medical Center of Yangzhou University (Yangzhou, China) were housed under conditions of a temperature of $20-24{ }^{\circ} \mathrm{C}$, a humidity of $55 \pm 5 \%$, and a $12 \mathrm{~h} / 12 \mathrm{~h}$ cycle of illumination, during which animals were free to eat and drink. Animal experiments were carried out in accordance with the ARRIVE (Animal Research: Reporting of in Vivo Experiments) guidelines ${ }^{62,63}$. All methods were endorsed by the Institutional Animal Care and Use Committee of China Pharmaceutical University.

Virus. AAVs used in this study were purchased from BrainVTA (Wuhan, China) and included AAV2/9-chat-cre-2a-EGFP, AAV2/9-chat-cre, AAV2/9-EF1 $\alpha$-DIO-Gcamp6m, AAV(2/9 or 2/Retro)Ef1 $\alpha$-DIO-hM4D(Gi)-mCherry, AAV(2/9 or 2/Retro)-Ef1 $\alpha$-DIO-hM3D(Gq)-mCherry, AAV(2/9 or 2/Retro)-Ef1 $\alpha$-DIO-eNpHR3.0-mCherry, AAV(2/9 or 2/Retro)-Ef1 $\alpha$-DIO-hChR2(H134R)-mCherry, AAV2/9-CAG-DIO-EGFP, AAV(2/9 or 2/Retro)-Ef1 $\alpha$-DIO-mCherry, AAV2/9-Ef1 $\alpha$-DIO-ACh2.0, AAV2/9-CaMKII $\alpha$-cre, AAV2/9-CaMKII $\alpha$-hM4D(Gi)-mCherry, AAV2/9-chat-EGFP and AAV2/9CaMKII $\alpha$-mCherry. AAV2/9-CAG-DIO-taCaspase3 was purchased from Taitool Bioscience (Shanghai, China). Virus titer was shown in Supplementary Table 1.

Drugs. CNO purchased from the Sigma-Aldrich (Missouri, USA) was dissolved in saline (2.5 mg/kg) for intraperitoneal injection while dissolved in artificial cerebrospinal fluid (ACSF, $3.0 \mu \mathrm{M} / 0.1 \mu \mathrm{l} /$ side) for stereotaxic injection. Benzethonium chloride purchased from Selleck Chemicals (Texas, USA) was dissolved in ACSF $(2.0 \mu \mathrm{g} / 0.1 \mu \mathrm{l} / \mathrm{side})$ for stereotaxic injection to inhibit $\alpha 4 \beta 2$ and $\alpha 7 \mathrm{nAChRs}$. Pirenzepine dihydrochloride purchased from the MedChemExpress (New Jersey, USA) was dissolved in $\operatorname{ACSF}(1.5 \mu \mathrm{g} / 0.1 \mu \mathrm{l} /$ side for stereotaxic injection or $20 \mu \mathrm{M}$ for electrophysiological recording) to block M1 mAChRs. 
Surgical procedures. For stereotaxic injection of AAVs, mice were anaesthetized with isoflurane $(1.5-5 \%)$ and administered ketoprofen $(5 \mathrm{mg} / \mathrm{kg}$ ) for pain management. The scalp was incised to expose the skull and connective tissue was gently scraped away. The AAVs were infused with a

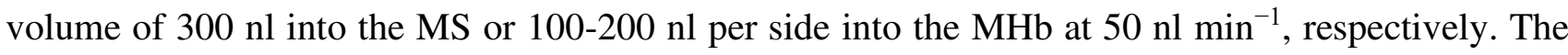
injection coordinates (relative to bregma, mm): MS (anterior/posterior (AP): +0.86 , medial/lateral (ML): 0, dorsal/ventral (DV): -4.20$)$, bilateral MHb (AP: $-1.82, \mathrm{ML}: \pm 0.20, \mathrm{DV}:-2.50)$ and bilateral CA3 (AP: -2.06, ML: $\pm 2.4, \mathrm{DV}:-2.2$ ). At the end of the infusion, the injectors remained at the site for $10 \mathrm{~min}$ to allow for virus diffusion. Skin covering the boreholes was sutured closed following surgery. For fiber photometry and optogenetic experiments, optical fibres ( $200 \mu \mathrm{m}$ core diameter, 0.37 NA; Inper, Hangzhou, China) threaded through $1.25 \mathrm{~mm}$-wide wide ceramic ferrules (Inper) were implanted into the $\mathrm{MS}$ or $\mathrm{MHb}\left(30^{\circ}\right.$ angle away from midline) 1 week before behavioral tasks, respectively, and secured to the mouse skull with dental cement. Stainless steel guide cannulas $(0.34$ mm core diameter; RWD, Shenzhen, China) were bilaterally implanted into the MHb with the same surgical approach for chemogenetic manipulations and drugs administrations. A total of 3-5 weeks were to ensure for viral expression before behavioral tasks. Animals infused for electrophysiology were maintained in their home cages for 6-8 weeks before recordings. Detailed strategy of virus injection can be found in Supplementary Table 1.

Immunohistochemistry. For tissue preparation, mice were anaesthetized and transcardially perfused with $50 \mathrm{ml}$ PBS followed by $4 \%$ paraformaldehyde (PFA) in PBS. The brain was removed and subsequently submerged into 4\% PFA overnight before being cryoprotected in $30 \%$ sucrose solution in PBS until they sank to the bottom of the container. Using a cryostat (CM3050S, Leica, Germany), the frozen brains were sectioned into $30-\mu \mathrm{m}$ coronal slices. Sections were washed in PBS three times (5 min each) and incubated with blocking solution (10\% normal donkey serum, $1 \%$ BSA, and $0.3 \%$ 
Triton X-100 in PBS) for $1 \mathrm{~h}$ at the room temperature. Sections were subsequently incubated with primary antibody in PBS overnight at $4{ }^{\circ} \mathrm{C}$. The following primary antibodies were used: sheep antiChAT (1:300, ab18736, Abcam, USA), rabbit anti-c-Fos (1:500, 226003, Synaptic Systems (SYSY), Germany) and mouse anti-CaMKII (1:300, ab22609, Abcam, USA). After washing in PBS three times (5 min each) and sections were incubated with fluorescein-labeled secondary antibody in PBS for $1 \mathrm{~h}$. The secondary antibodies were used: donkey anti-sheep Alexa Fluor 488 or 594 (1:1000, ab 150177 or ab 150180, Abcam, USA), donkey anti-rabbit Alexa Fluor 594 (1:1000, ab 150076, Abcam, USA) and donkey anti-mouse Alexa Fluor 594 (1:1000, ab 150108, Abcam, USA). After washing in PBS and staining with DAPI (C0065, Solarbio, Beijing, China), brain sections were mounted on positively charged slides with prolong anti-fade medium. Fluorescent images were taken using a laser confocal microscope (AiryScan LSM800, Zeiss, Germany) or fluorescence microscope (DM2000, Leica, Germany). All images were taken in roughly the same imaging area and images were processed using NIH ImageJ software. Sample size was based on reports in related literature and was not predetermined by calculation.

In vitro electrophysiological recording. The methods of brain slice preparation and electrophysiological recordings were similar to those in previous studies ${ }^{64,65,66}$. In brief, after the full expressions of AAVs, mice were anaesthetized with isoflurane and the brains were rapidly removed and chilled in ice-cold sucrose solution containing (in $\mathrm{mM}$ ): $40 \mathrm{NaCl}, 4.5 \mathrm{KCl}, 1.25 \mathrm{NaH}_{2} \mathrm{PO}_{4}, 25$ $\mathrm{NaHCO}_{3}, 148.5$ sucrose, 10 glucose, 1 ascorbic acid, $3 \mathrm{Na}$ Pyruvate, $3 \mathrm{Myo}$ Inositol, $0.5 \mathrm{CaCl}_{2}$ and 7 $\mathrm{MgSO}_{4}, \mathrm{pH} 7.3,315 \mathrm{mOsm}$. Coronal brain slices $(300 \mu \mathrm{m})$ containing MS or MHb were prepared in the same ice-cold sucrose solution using a vibratome (VT-1200s, Leica, Germany). Slices were then incubated in warm $\left(32-34{ }^{\circ} \mathrm{C}\right)$ sucrose solution for $30 \mathrm{~min}$, and transferred to ACSF composed of (in mM): $125 \mathrm{NaCl}, 4.5 \mathrm{KCl}, 1.25 \mathrm{NaH}_{2} \mathrm{PO}_{4}, 25 \mathrm{NaHCO}_{3}, 15$ sucrose, 15 glucose, $2.5 \mathrm{CaCl}_{2}$ and 1.3 
$\mathrm{MgSO}_{4}, \mathrm{pH} 7.3,315 \mathrm{mOsm}$, and allowed to cool to room temperature before electrophysiological recording. All solutions were continuously bubbled with 95\% O2/5\% CO2. Electrophysiological recordings were made using a MultiClamp700B amplifier and PClamp software (Molecular Devices, USA). The data were low-pass filtered at $2 \mathrm{kHz}$ and digitized at $10 \mathrm{kHz}$ with Digidata 1440 (Molecular Devices, USA). During recording, slices were submerged in normal, oxygenated ACSF and superfused $(2 \mathrm{ml} / \mathrm{min})$ at temperature $\left(32-34{ }^{\circ} \mathrm{C}\right)$.

To identify MS cholinergic neurons, after expression of AAV2/9-chat-cre-2a-EGFP, spontaneous action potentials (APs) of EGFP-labeled neurons in the MS were firstly recorded. Then APs were recorded with intracellular current injection $(+80 \mathrm{pA}$ or $-80 \mathrm{pA}$, respectively) by whole-cell current clamp. The pipette (3-5 M $\Omega$ ) was pulled by a micropipette puller (P-97, Sutter instrument) and filled with the internal solution (in mM: $105 \mathrm{~K}$-gluconate, $5 \mathrm{NaCl}, 10$ HEPES, $2 \mathrm{MgATP}, 0.5 \mathrm{NaGTP}$, and 0.2 EGTA, pH 7.3, 290 mOsm).

To confirm the functionality of expressed NpHR and $h M 3 D(G q)$, spontaneous firing of NpHRor hM3D(Gq)-expressing neurons in the MS were recorded by cell-attached voltage clamp. Following a 3-min stabilization period, cell-attached voltage clamp was performed. And then, after 1 min continuous recording, yellow-light $(589 \mathrm{~nm})$ was delivered to brain slices or $\mathrm{CNO}(5 \mu \mathrm{M})$ was added to ACSF solution for the following recording.

To record EPSCs of MHb neurons, AAV2/9-CaMKII $\alpha$-mCherry and AAV2/9-chat-EGFP were injected into the $\mathrm{MHb}$. $\mathrm{MHb}$ glutamatergic neurons that corelease glutamate and acetylcholine $\left(\mathrm{MHb}^{\mathrm{CaMKII} \alpha+\mathrm{ChAT}+}\right)$ were labeled with chat-EGFP and CaMKII $\alpha-m$ Cherry. MHb glutamatergic neurons without releasing acetylcholine $\left(\mathrm{MHb}^{\mathrm{CaMKII} \alpha+\mathrm{ChAT}_{-}}\right)$were labeled with CaMKII $\alpha-\mathrm{mCherry}$ alone. EPSCs were recorded by whole-cell voltage-clamp. Patch electrodes (3-5 M $\Omega$ ) were filled with pipette solution contained (in $\mathrm{mM}$ ): $132.5 \mathrm{Cs}$-gluconate, $17.5 \mathrm{CsCl}, 2 \mathrm{MgCl}_{2}, 0.5$ EGTA, $10 \mathrm{HEPES}$, 
4 ATP, 5 QX-314, pH 7.3, 290-300 mOsm). For voltage-clamp recordings, EPSCs were recorded at $-70 \mathrm{mV}$. After expressions of AAV2/9-chat-cre and AAV2/9-Ef1 $\alpha$-DIO-ChR2-mCherry in the MS, for the photostimulation of ChR2-expression axon terminals, a blue light pulse was emitted from a Lambda DG-4 (Sutter, USA) under the control of Digidata 1440. A Lambda DG-4 Wavelength Switcher was used to deliver different wavelengths of light through the $40 \times$ objective. Following a 3 min stabilization period, sEPSCs of MHb glutamatergic neurons were firstly continuous recorded for $1 \mathrm{~min}$, and then blue-light $(465 \mathrm{~nm}, 5 \mathrm{~ms}, 20 \mathrm{~Hz}$ ) was delivered to the MHb slices for the following light-evoked EPSC recording. Additionally, M1 mAChRs antagonist pirenzepine dihydrochloride (20 $\mu \mathrm{M})$ was added to ACSF solution for the last recording. Amplitude and frequency of EPSCs were analyzed with Clampfit 11.1 software.

Apparatus for fiber photometry and optogenetic experiments. After the expressions of AAV2/9EF1 $\alpha$-DIO-Gcamp6m in the MS and AAV2/9-Ef1 $\alpha$-DIO-ACh2.0 in the MHb, fiber photometry system (Inper) was used to record GCaMP and acetylcholine fluorescence signals. Firstly, laser beam from a 470-nm laser (B1470; Inper) was reflected by a dichroic mirror (MD498; Thorlabs, USA), focused by a $\mathrm{x} 10$ objective lens $(\mathrm{NA}=0.3$; Olympus, Japan) and then coupled to an optical commutator (Inper). An optical fiber ( $230 \mu \mathrm{m}$ O.D., NA $=0.37,1 \mathrm{~m}$ long) guided the light between the commutator and the implanted optical fiber. The laser power was adjusted at the tip of optical fiber to a low level of 0.01-0.02 $\mathrm{mW}$, to minimize bleaching. Then the fluorescence was bandpass filtered (MF525-39, Thorlabs) and focused by a 20 times objective lens and the fluorescence signal was collected by CMOS camera. The end of the fiber was imaged at the frame rate from 1-320. The ROI area size and mean value were set through Inper Studio. $470 \mathrm{~nm}$ and $410 \mathrm{~nm}$ light sources were given alternately, and 410 nm was used as the control. 
For optogenetic experiments, light was delivered to the MS or the MHb via a laser (Inper) connected through a ceramic mating sleeve (Inper) to the ferrule implanted on the mice. A 589-nm laser was used to inhibit MS cholinergic neurons or their terminals at bilateral $\mathrm{MHb}$ in $\mathrm{NpHR}$ expressing mice. Yellow light intensity was delivered at $10 \mathrm{~mW}$. Meanwhile, a 465-nm laser was used to activate MS cholinergic neurons or their terminals at bilateral $\mathrm{MHb}$ in $\mathrm{ChR} 2$-expressing mice. 10 $40 \mathrm{~ms}$ blue light pulses were delivered at $20 \mathrm{~Hz}$.

\section{Behavioral protocols}

Visual aversion task. The apparatus consisted of a two-compartment $(50 \mathrm{~cm} \times 25 \mathrm{~cm} \times 25 \mathrm{~cm}$ for each chamber) blue plastic box. The dividing wall was made from non-transparent blue plastic with a rectangular opening (10 $\mathrm{cm}$ in width) in the middle to enable mice free access to each chamber. Each chamber was filled with four digital screens $(9.7$-inch, resolution: $2048 \times 1536$ dpi) on its three walls (except dividing wall). In the task, mice were first habituated to the two-chamber apparatus for $10 \mathrm{~min}$ while all digital screens were off. Then in test phase, four digital screens in each chamber displayed one of three images: a blank image (image format: JPG), a static stripe image (image format: JPG) and the same stripe image that was moving (named as dynamic stripe image, image format: GIF, five frames per image, all frame delays 3 with GIF Movie Gear software), and mice were placed in the twochamber apparatus for a 5-min real-time place preference (RTPP) test. Visual aversion behavior for an image was defined by the percentage of time spent in each image chamber.

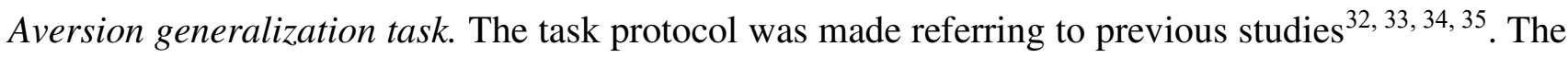
same two-chamber apparatus was used in aversion generalization task. The task involved five phases, including two habituation phases, one training phase, and two test phases. In habituation phase 1, mice were placed in the apparatus with all eight digital screens off for a 10-min habituation. $1 \mathrm{~h}$ later, in habituation phase 2 , mice were placed in the apparatus that one chamber showed same blank images 
whereas the other chamber showed same static stripes image on screens for a 10 min habituation. $1 \mathrm{~h}$ later, in training phase, mice were placed in the apparatus with all two chambers displaying corresponding dynamic stripe images identified as aversive images for a 10-min visual stimulation. After a 1-h delay in test phase 1, mice were placed again in the apparatus that one chamber showed blank images whereas the other chamber showed static stripe images for a 5-min RTPP-test. After a 4-h delay in test phase 2, mice were placed in the apparatus for a 5-min RTPP-test in the same condition with test phase 1. Visual aversion behavior was defined by the percentage of time spent in each chamber.

Open field test (OFT). Each mouse was placed in the open field chamber (a plastic box with dimensions of $50 \mathrm{~cm} \times 50 \mathrm{~cm} \times 40 \mathrm{~cm}$ divided into 144 squares), and time spent in the center was assessed for 5 min using video camera and software to record and analyze paths. Anxiety-like behavior was defined by the time spent in the central zone.

Elevated plus-maze (EPM) test. Each mouse was placed in the center of the EPM and videotaped for $5 \mathrm{~min}$. The time spent in two open arms $(30 \mathrm{~cm} \times 5 \mathrm{~cm})$ and two closed arms $(30 \mathrm{~cm} \times 5 \mathrm{~cm} \times 20 \mathrm{~cm})$ were determined from the video recording by blinded observers. Anxiety-like behavior was defined by the time spent in the open arms.

Grip strength test (GST). Forelimb grip strength was measured as tension force using a computerized grip strength meter (GSM) ${ }^{67}$. Each mouse was lifted over the baseplate by its tail, and its forepaws allowed to grasp the steel grip. The tail of each mouse was then placed in the center of alignment tool and the mouse was then gently pulled backward by the tail until its grip was released. The GSM was then used to measure the maximal force $(\mathrm{N})$ before the mouse released the bar. Three trials were performed for each mouse with a 2-min resting period between trials. 
Novel object recognition test (NORT). NORT is accomplished within three days and consists of a habituation, training, and testing session. The first day was the habituation session in which mice were allowed to move freely in a rectangular plastic box $(50 \mathrm{~cm} \times 50 \mathrm{~cm} \times 40 \mathrm{~cm})$. On the second day (training session), the mice were allowed to explore two identical objects for 5 min. On the third day (testing session), one of the objects was changed to a new object (different color and shape), and the mice were allowed to explore for $5 \mathrm{~min}$. The time spent exploring, defined as mouth, nose, or paw less than $2 \mathrm{~cm}$ away from the object, was recorded by the computer. The discrimination index, or the difference in exploration time for novel object minus the familiar object divided by the total amount of exploration of both objects, was assessed.

Morris water maze (MWM) test. Each mouse was placed in a round pool (1.2 m diameter, $0.5 \mathrm{~m}$ height) filled with water to a $0.3 \mathrm{~m}$ depth at a temperature of $25^{\circ} \mathrm{C}$. An escape platform with a diameter of 9 $\mathrm{cm}$ was placed at the midpoint of any quadrant of the pool, and visual cues were located around the room. The test consisted of five days training period with visible and hidden platform. The escape platform always stayed at a fixed position during the entire duration of training (5 days). On every trial, mice were put into the pool facing the pool wall. The visible platform training sessions were performed on days 1 and 2. During visible training trials, a small flag was affixed to the platform that was $1 \mathrm{~cm}$ below the surface of the water. Hidden platform training sessions in which the small flag was removed but the position of the platform remained the same were performed on days 3-5. There were four trials per day, each lasting $90 \mathrm{~s}$, with an intertrial interval of 1 hour. Once on the platform, the trial end after $10 \mathrm{~s}$. If the mouse failed to find the platform after $90 \mathrm{~s}$, it would gently guide to the platform and left for $30 \mathrm{~s}$ before being removed. The time to find the platform (escape latency) was compared. On the final day (day 6), during the probe trial, mice were allowed to swim for $90 \mathrm{~s}$ while the platform is taken away. The number of times of each individual mouse passing the escape platform location and the time 
spent in the target quadrant were recorded and processed using video tracking device and computerequipped analytics management system.

$Y$-maze test. Spontaneous alternation behavior was evaluated in a symmetrical Y-maze $(3 \mathrm{arms}, 40 \mathrm{~cm}$ $\times 9 \mathrm{~cm} \times 16 \mathrm{~cm}$ ). Arm choices (all four paws entering one arm) were recorded during an 8-min exploration trial in the Y-shaped maze by a video camera. Alternation was defined by recording the order of the visited arms (A, B, or C). Overlapping triplets of 3-arm visits was counted as one complete spontaneous alternation. The percentage of spontaneous alternation was calculated according to the following calculation: (number of spontaneous alternation) / (total number of arm visits -2 ).

Statistical analysis. All experiments and data analyses were conducted blinded, including the immunohistochemistry and behavioral analyses. SPSS (version 17.0) was used for statistical analysis as indicated in the figure legends. All data are shown as mean \pm standard error of mean (SEM). Statistical significance was assessed by two-tailed unpaired Student's $t$ test or one-way ANOVA followed by a Tukey's post-hoc analysis for multiple comparisons. $P<0.05$ was considered statistically significant. $* P<0.05, * * P<0.01$, $* * * P<0.001$, n.s.: $P>0.05$.

2




\section{References}

1. Shalev A, Liberzon I, Marmar C. Post-Traumatic Stress Disorder. New Engl J Med 376, 24592469 (2017).

2. LeDoux JE. Emotion circuits in the brain. Annu Rev Neurosci 23, 155-184 (2000).

3. Marsicano $\mathrm{G}$, et al. The endogenous cannabinoid system controls extinction of aversive memories. Nature 418, 530-534 (2002).

4. Onat S, Buchel C. The neuronal basis of fear generalization in humans. Nat Neurosci 18, 18111818 (2015).

5. Parsons RG, Ressler KJ. Implications of memory modulation for post-traumatic stress and fear disorders. Nat Neurosci 16, 146-153 (2013).

6. Yehuda R, et al. Post-traumatic stress disorder. Nat Rev Dis Primers 1, (2015).

7. McGuire JF, Orr SP, Essoe JKY, McCracken JT, Storch EA, Piacentini J. Extinction learning in childhood anxiety disorders, obsessive compulsive disorder and post-traumatic stress disorder: implications for treatment. Expert Rev Neurother 16, 1155-1174 (2016).

8. Drew MR, Huckleberry KA. Modulation of Aversive Memory by Adult Hippocampal Neurogenesis. Neurotherapeutics 14, 646-661 (2017).

9. Ozawa T, et al. A feedback neural circuit for calibrating aversive memory strength. Nat Neurosci 20, 90-97 (2017).

10. Vanz F, Bicca MA, Linartevichi VF, Giachero M, Bertoglio LJ, de Lima TCM. Role of dorsal hippocampus kappa opioid receptors in contextual aversive memory consolidation in rats. Neuropharmacology 135, 253-267 (2018).

11. Chen JY, Campos CA, Jarvie BC, Palmiter RD. Parabrachial CGRP Neurons Establish and Sustain Aversive Taste Memories. Neuron 100, 891-+ (2018).

12. Slouzkey I, Rosenblum K, Maroun M. Memory of Conditioned Taste Aversion Is Erased by Inhibition of PI3K in the Insular Cortex. Neuropsychopharmacol 38, 1143-1153 (2013).

13. Hampel H, et al. The cholinergic system in the pathophysiology and treatment of Alzheimer's disease. Brain 141, 1917-1933 (2018).

14. Raza SA, et al. HIPP neurons in the dentate gyrus mediate the cholinergic modulation of background context memory salience. Nat Commun 8, 189 (2017).

15. Ballinger EC, Ananth M, Talmage DA, Role LW. Basal Forebrain Cholinergic Circuits and Signaling in Cognition and Cognitive Decline. Neuron 91, 1199-1218 (2016). 
16. Jiang L, et al. Cholinergic Signaling Controls Conditioned Fear Behaviors and Enhances Plasticity of Cortical-Amygdala Circuits. Neuron 90, 1057-1070 (2016).

17. Mesulam MM, Mufson EJ, Levey AI, Wainer BH. Cholinergic innervation of cortex by the basal forebrain: cytochemistry and cortical connections of the septal area, diagonal band nuclei, nucleus basalis (substantia innominata), and hypothalamus in the rhesus monkey. J Comp Neurol 214, 170-197 (1983).

18. Woolf NJ. Cholinergic systems in mammalian brain and spinal cord. Prog Neurobiol 37, 475$524(1991)$.

19. Jiang YY, Zhang Y, Cui S, Liu FY, Yi M, Wan Y. Cholinergic neurons in medial septum maintain anxiety-like behaviors induced by chronic inflammatory pain. Neurosci Lett 671, 712 (2018).

20. Zhang Y, et al. Inhibiting medial septal cholinergic neurons with DREADD alleviated anxietylike behaviors in mice. Neurosci Lett 638, 139-144 (2017).

21. Staib JM, Della Valle R, Knox DK. Disruption of medial septum and diagonal bands of Broca cholinergic projections to the ventral hippocampus disrupt auditory fear memory. Neurobiol Learn Mem 152, 71-79 (2018).

22. Agetsuma M, et al. The habenula is crucial for experience-dependent modification of fear responses in zebrafish. Nat Neurosci 13, 1354-1356 (2010).

23. McLaughlin I, Dani JA, De Biasi M. The medial hahenula and interpeduncular nucleus circuitry is critical in addiction, anxiety, and mood regulation. $J$ Neurochem 142, 130-143 (2017).

24. Yamaguchi T, Danjo T, Pastan I, Hikida T, Nakanishi S. Distinct Roles of Segregated Transmission of the Septo-Habenular Pathway in Anxiety and Fear. Neuron 78, 537-544 (2013).

25. Boulos LJ, et al. Mu opioid receptors in the medial habenula contribute to naloxone aversion. Neuropsychopharmacology 45, 247-255 (2020).

26. Soria-Gomez E, et al. Habenular CB1 Receptors Control the Expression of Aversive Memories. Neuron 88, 306-313 (2015).

27. Paul S, Jeon WK, Bizon JL, Han JS. Interaction of basal forebrain cholinergic neurons with the glucocorticoid system in stress regulation and cognitive impairment. Front Aging Neurosci 7, $43(2015)$. 
28. Shalev L, Paz R, Avidan G. Visual Aversive Learning Compromises Sensory Discrimination. The Journal of neuroscience : the official journal of the Society for Neuroscience 38, 27662779 (2018).

29. Blechert J, Michael T, Vriends N, Margraf J, Wilhelm FH. Fear conditioning in posttraumatic stress disorder: evidence for delayed extinction of autonomic, experiential, and behavioural responses. Behav Res Ther 45, 2019-2033 (2007).

30. Lopresto D, Schipper P, Homberg JR. Neural circuits and mechanisms involved in fear generalization: Implications for the pathophysiology and treatment of posttraumatic stress disorder. Neurosci Biobehav Rev 60, 31-42 (2016).

31. O'Malley KR, Waters AM. Attention avoidance of the threat conditioned stimulus during extinction increases physiological arousal generalisation and retention. Behav Res Ther 104, 51-61 (2018).

32. Aqrabawi AJ, Kim JC. Hippocampal projections to the anterior olfactory nucleus differentially convey spatiotemporal information during episodic odour memory. Nature communications $\mathbf{9}$, 2735 (2018).

33. Shen CJ, et al. Cannabinoid CB1 receptors in the amygdalar cholecystokinin glutamatergic afferents to nucleus accumbens modulate depressive-like behavior. Nature medicine 25, 337349 (2019).

34. Song J, et al. Crucial role of feedback signals from prelimbic cortex to basolateral amygdala in the retrieval of morphine withdrawal memory. Science advances 5, eaat3210 (2019).

35. $\mathrm{Xu} \mathrm{Y,} \mathrm{et} \mathrm{al.} \mathrm{Identification} \mathrm{of} \mathrm{a} \mathrm{neurocircuit} \mathrm{underlying} \mathrm{regulation} \mathrm{of} \mathrm{feeding} \mathrm{by} \mathrm{stress-related}$ emotional responses. Nature communications 10, 3446 (2019).

36. Levey AI, Kitt CA, Simonds WF, Price DL, Brann MR. Identification and localization of muscarinic acetylcholine receptor proteins in brain with subtype-specific antibodies. $J$ Neurosci 11, 3218-3226 (1991).

37. Gotti C, Zoli M, Clementi F. Brain nicotinic acetylcholine receptors: native subtypes and their relevance. Trends Pharmacol Sci 27, 482-491 (2006).

38. Koppensteiner P, Galvin C, Ninan I. Development- and experience-dependent plasticity in the dorsomedial habenula. Mol Cell Neurosci 77, 105-112 (2016).

39. Jing M, et al. A genetically encoded fluorescent acetylcholine indicator for in vitro and in vivo studies. Nat Biotechnol 36, 726-737 (2018).

40. Ren J, et al. Habenula "cholinergic" neurons co-release glutamate and acetylcholine and activate postsynaptic neurons via distinct transmission modes. Neuron 69, 445-452 (2011). 
41. Berg H, et al. Salience and central executive networks track overgeneralization of conditionedfear in post-traumatic stress disorder. Psychol Med, 1-10 (2020).

42. De Bundel D, Zussy C, Espallergues J, Gerfen CR, Girault JA, Valjent E. Dopamine D2 receptors gate generalization of conditioned threat responses through mTORC1 signaling in the extended amygdala. Mol Psychiatry 21, 1545-1553 (2016).

43. Kaczkurkin AN, et al. Neural Substrates of Overgeneralized Conditioned Fear in PTSD. Am J Psychiatry 174, 125-134 (2017).

44. Lissek S, Kaczkurkin AN, Rabin S, Geraci M, Pine DS, Grillon C. Generalized anxiety disorder is associated with overgeneralization of classically conditioned fear. Biol Psychiatry 75, 909915 (2014).

45. Rudy JW, Huff NC, Matus-Amat P. Understanding contextual fear conditioning: insights from a two-process model. Neurosci Biobehav Rev 28, 675-685 (2004).

46. Izquierdo I, Furini CR, Myskiw JC. Fear Memory. Physiol Rev 96, 695-750 (2016).

47. King AJ. Visual influences on auditory spatial learning. Philos Trans $R$ Soc Lond B Biol Sci 364, 331-339 (2009).

48. Csaszar N, Kapocs G, Bokkon I. A possible key role of vision in the development of schizophrenia. Rev Neurosci 30, 359-379 (2019).

49. Yilmaz Balban M, et al. Human Responses to Visually Evoked Threat. Curr Biol, (2020).

50. Loprinzi PD, Codey K. Influence of visual acuity on anxiety, panic and depression disorders among young and middle age adults in the United States. $J$ Affect Disord 167, 8-11 (2014).

51. Gritti I, Henny P, Galloni F, Mainville L, Mariotti M, Jones BE. Stereological estimates of the basal forebrain cell population in the rat, including neurons containing choline acetyltransferase, glutamic acid decarboxylase or phosphate-activated glutaminase and colocalizing vesicular glutamate transporters. Neuroscience 143, 1051-1064 (2006).

52. Bao H, et al. Long-Range GABAergic Inputs Regulate Neural Stem Cell Quiescence and Control Adult Hippocampal Neurogenesis. Cell Stem Cell 21, 604-617 e605 (2017).

53. Robinson J, et al. Optogenetic Activation of Septal Glutamatergic Neurons Drive Hippocampal Theta Rhythms. J Neurosci 36, 3016-3023 (2016).

54. Kobayashi Y, et al. Genetic dissection of medial habenula-interpeduncular nucleus pathway function in mice. Frontiers in behavioral neuroscience 7, 17 (2013). 
55. Lecourtier L, Neijt HC, Kelly PH. Habenula lesions cause impaired cognitive performance in rats: implications for schizophrenia. The European journal of neuroscience 19, 2551-2560 (2004).

56. Sharma VK, et al. Molecular and functional identification of $\mathrm{m} 1$ muscarinic acetylcholine receptors in rat ventricular myocytes. Circ Res 79, 86-93 (1996).

57. Wu W, Li H, Liu Y, Huang X, Chen L, Zhai H. Involvement of insular muscarinic cholinergic receptors in morphine-induced conditioned place preference in rats. Psychopharmacology (Berl) 231, 4109-4118 (2014).

58. Coates KM, Flood P. Ketamine and its preservative, benzethonium chloride, both inhibit human recombinant alpha7 and alpha4beta2 neuronal nicotinic acetylcholine receptors in Xenopus oocytes. Br J Pharmacol 134, 871-879 (2001).

59. Zhang J, et al. Presynaptic Excitation via GABAB Receptors in Habenula Cholinergic Neurons Regulates Fear Memory Expression. Cell 166, 716-728 (2016).

60. Zhao-Shea R, et al. Increased CRF signalling in a ventral tegmental area-interpeduncular nucleus-medial habenula circuit induces anxiety during nicotine withdrawal. Nat Commun $\mathbf{6}$, 6770 (2015).

61. Molas S, DeGroot SR, Zhao-Shea R, Tapper AR. Anxiety and Nicotine Dependence: Emerging Role of the Habenulo-Interpeduncular Axis. Trends Pharmacol Sci 38, 169-180 (2017).

62. Kilkenny C, Browne W, Cuthill IC, Emerson M, Altman DG, Group NCRRGW. Animal research: reporting in vivo experiments: the ARRIVE guidelines. Br J Pharmacol 160, 15771579 (2010).

63. McGrath JC, Lilley E. Implementing guidelines on reporting research using animals (ARRIVE etc.): new requirements for publication in BJP. Br J Pharmacol 172, 3189-3193 (2015).

64. Ma T, et al. Bidirectional and long-lasting control of alcohol-seeking behavior by corticostriatal LTP and LTD. Nat Neurosci 21, 373-383 (2018).

65. Zou WJ, et al. A discrete serotonergic circuit regulates vulnerability to social stress. Nat Commun 11, 4218 (2020).

66. Liang HY, et al. nNOS-expressing neurons in the vmPFC transform pPVT-derived chronic pain signals into anxiety behaviors. Nat Commun 11, 2501 (2020).

67. Liu K, et al. Lhx6-positive GABA-releasing neurons of the zona incerta promote sleep. Nature 548, 582-587 (2017). 


\section{Acknowledgements}

This work was supported by grants from the National Natural Science Foundation of China (81773714 and 81573413 to Hao Hong) and the "Double First-Class" University Project (CPU2018GF/GY**). We acknowledge technical support by the Public Experimental Pharmacology Platform of China Pharmaceutical University, and appreciate Dr. Miranda N. Reed and Prof. Vishnu Suppiramaniam from Auburn University for their conceptual suggestion and language assistance.

\section{Author contributions}

H.H., S.-S.T. and R.-H.M. carried out the study conceptualization and experimental design. R.-H.M. performed apoptosis, chemogenetic, and optogenetic studies. R.-H.M. and X.-M.H. performed synaptic tracing and in vivo fiber photometry. X.-M.H. and H.W. performed virus vectors injections and immunohistochemistry. Y.L. and D.-H.Y. performed imaging and cell counting. H.H. and R.-H.M. wrote the manuscript. S.-S.T. and H.W. revised the manuscript. All authors contributed to the data analysis and presentation in the paper.

\section{Competing interests}

The authors declare no competing interests. 
Figure. 1

a

AAV-chat-cre-2a-EGFP

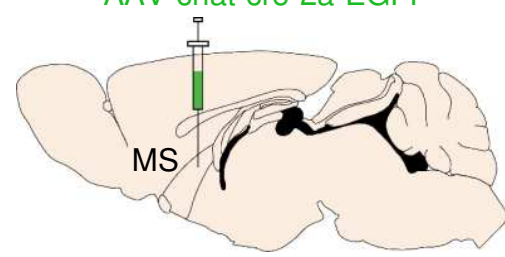

b

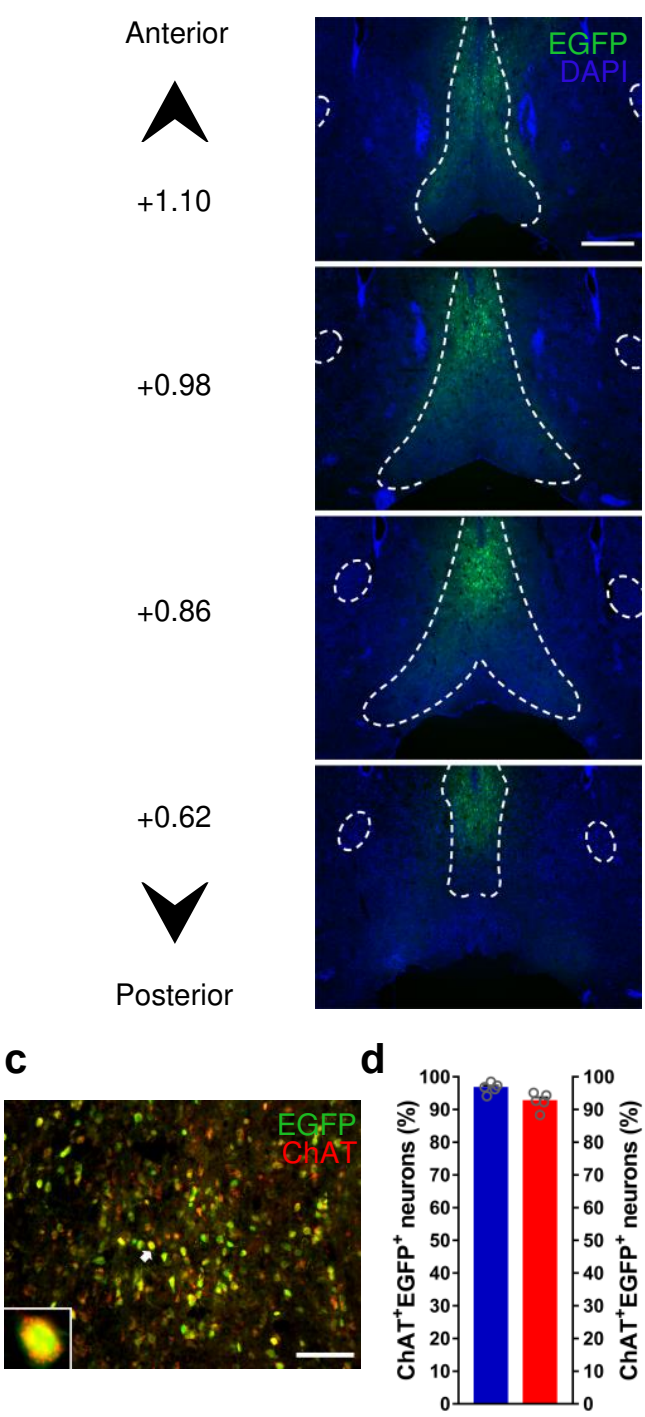

e

f

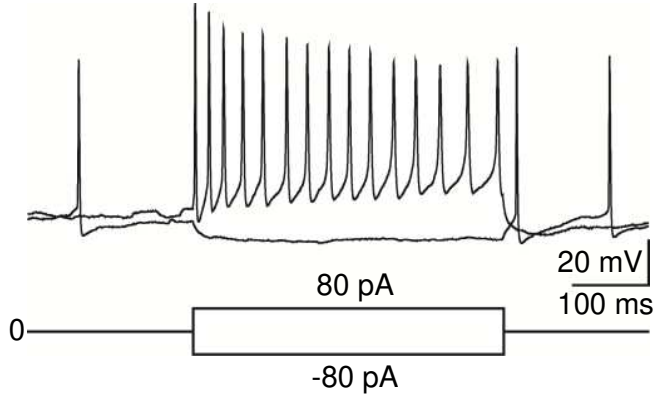

g

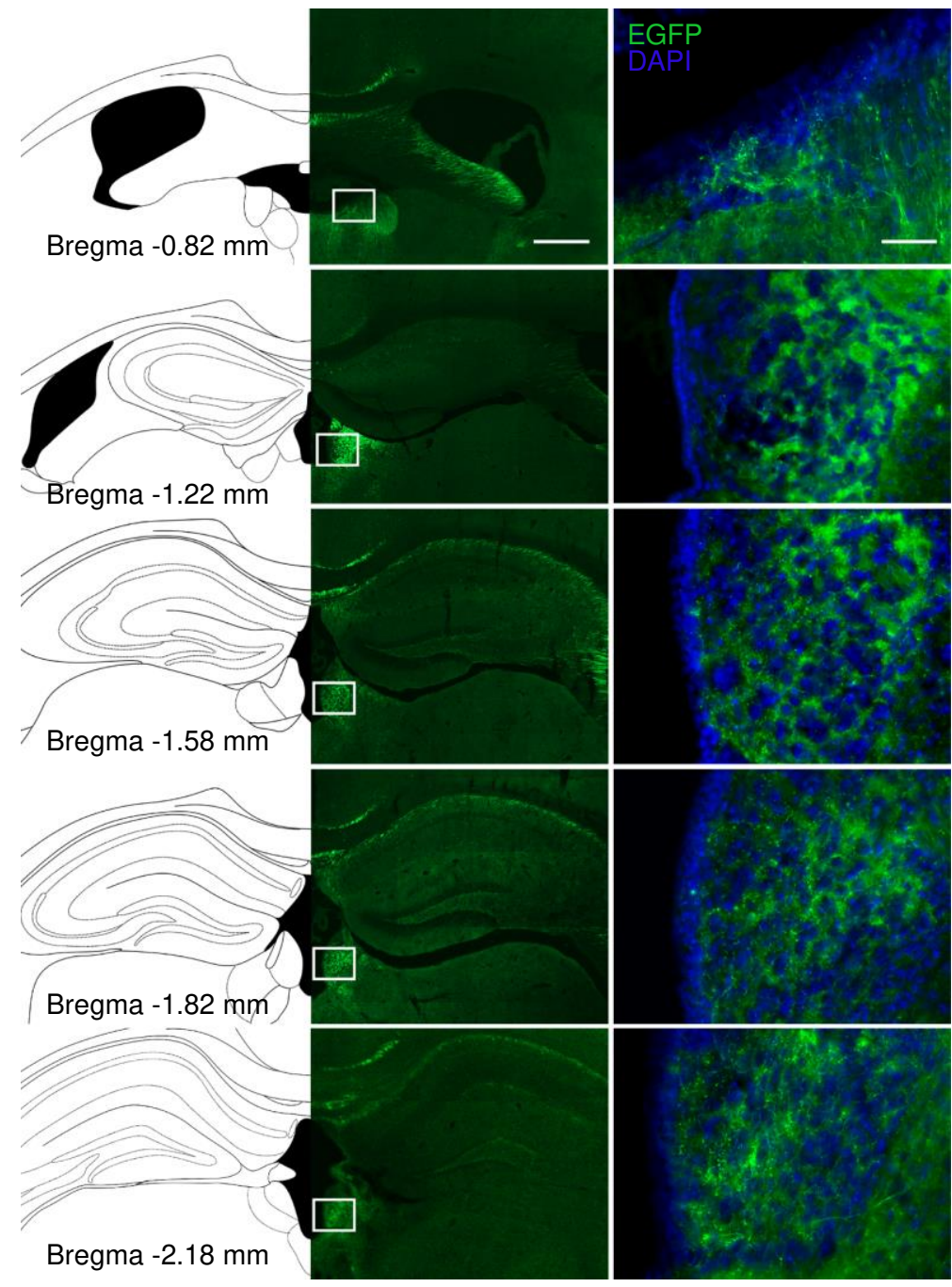

Fig. 1 MS provides strong cholinergic projections to the MHb. a Schematic diagram of virus injection of AAV-chat-cre-EGFP into the MS to track its cholinergic projections. b Representative images of AAV-chat-cre-EGFP expression in the MS three weeks after virus injection. The numbers 


836

7

correspond to anterior-posterior distance (in $\mathrm{mm}$ ) from bregma. c Representative image of EGFPlabeled cholinergic neurons (green) stained with anti-ChAT (red) in the MS by immunohistochemistry. Inset, magnification of targeted neuron marked with a white arrow. d Quantification of the percentage of $\mathrm{ChAT}^{+} \mathrm{EGFP}^{+}$cells (blue) relative to all EGFP-labeled cholinergic neurons in the MS. Quantification of the percentage of $\mathrm{ChAT}^{+} \mathrm{EGFP}^{+}$cells (red) relative to all anti-ChAT neurons in the MS. $n=5$, four sections per mouse. e Representative image of EGFP-labeled MS cholinergic neurons in whole-cell patch-clamp recording after a 6-8 weeks expression of AAV-chat-cre-EGFP. f Response of EGFP-labeled MS cholinergic neurons to intracellular current steps during whole-cell recording. Depolarizing current: +80 pA; hyperpolarization current: -80 pA. g Representative whole-brain mapping of EGFP-labeled cholinergic projections from the MS to the MHb. Scale bar, $400 \mu \mathrm{m}(\mathbf{b})$, $100 \mu \mathrm{m}(\mathbf{c}), 50 \mu \mathrm{m}(\mathbf{e}), 400 \mu \mathrm{m}(\mathbf{g}$, middle $)$ and $50 \mu \mathrm{m}(\mathbf{g}$, right). Data are mean \pm s.e.m. Additional statistical information can be found in Supplementary Table 2. 
Figure. 2

848

849

850

851

852

853

854

855

856

857

858

859

860

861

862

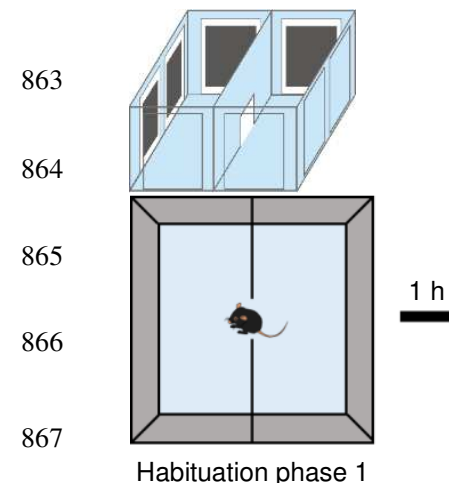

868

869

870

871

872 a
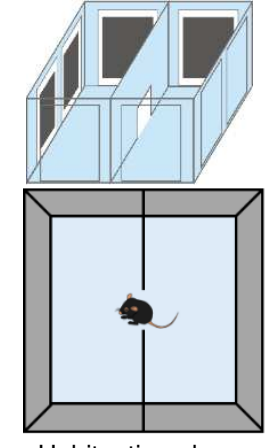

Habituation phase

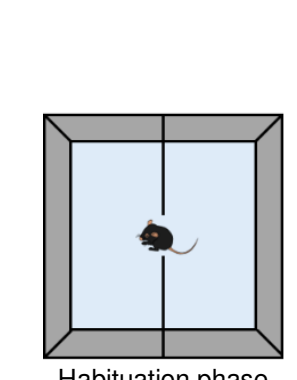

Habituation phase

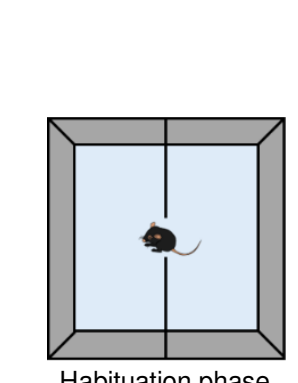

Habituation phase d

e

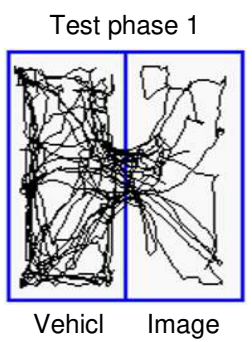

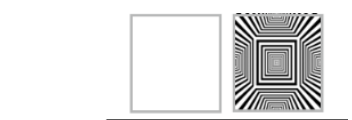

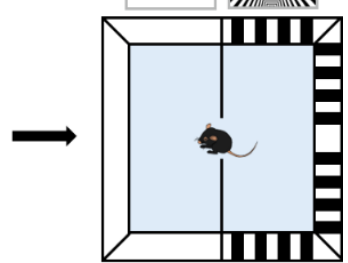

Test phase
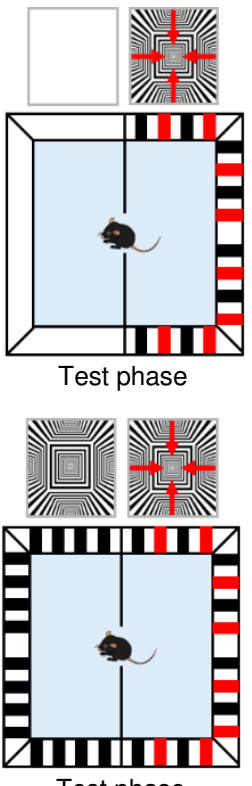

Test phase b
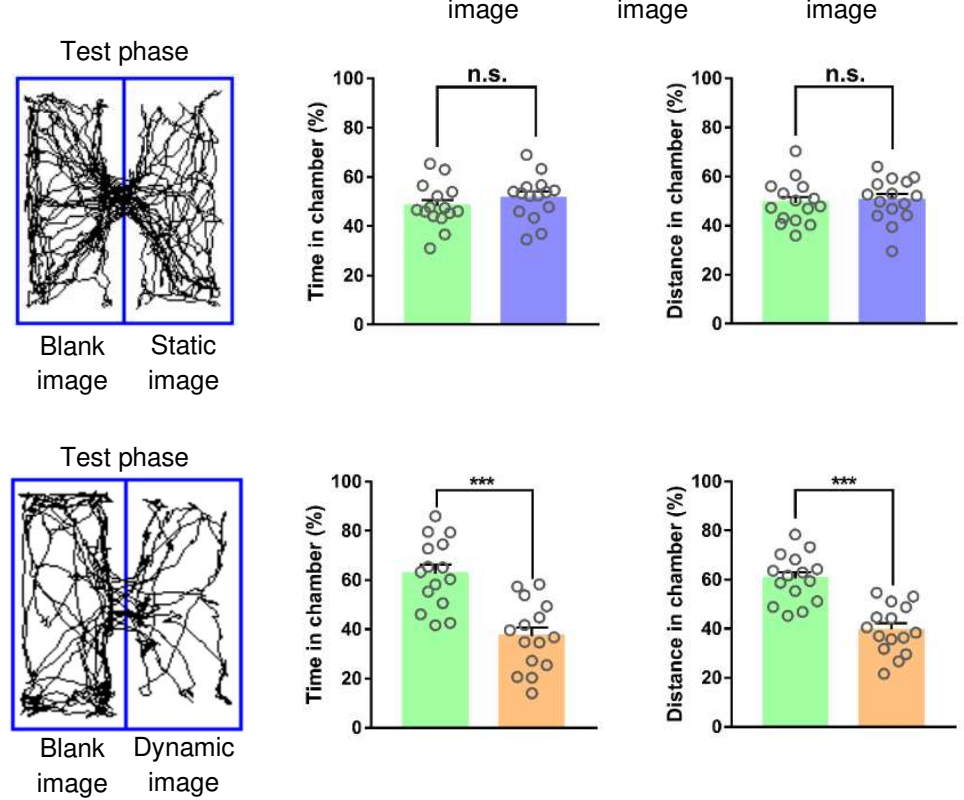
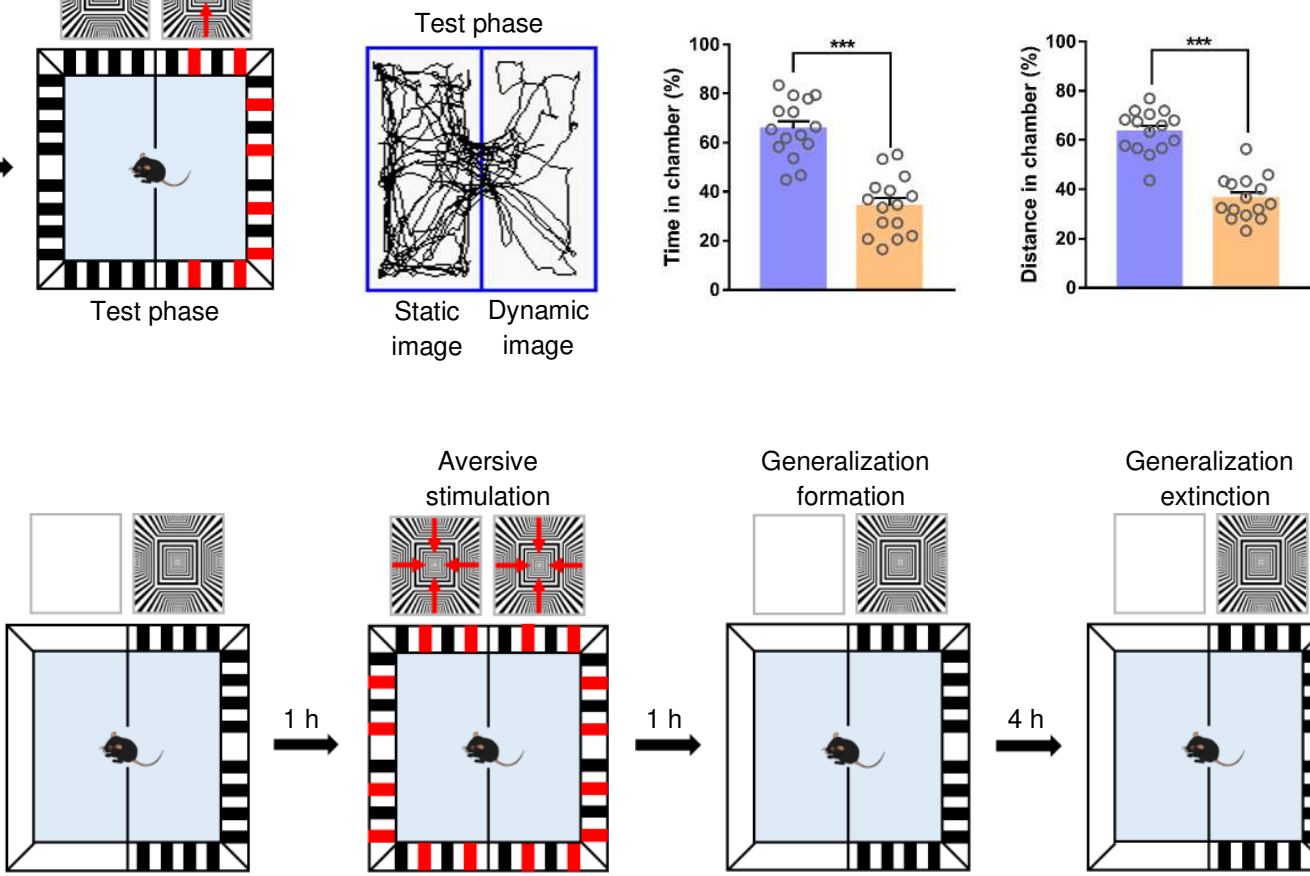

Habituation phase 2

$\stackrel{\mathrm{h}}{\longrightarrow}$

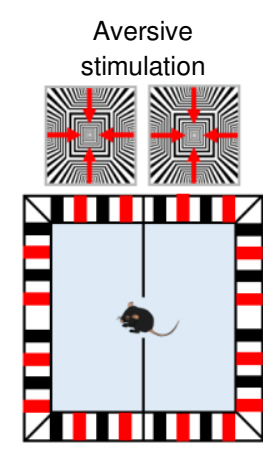

Training phase

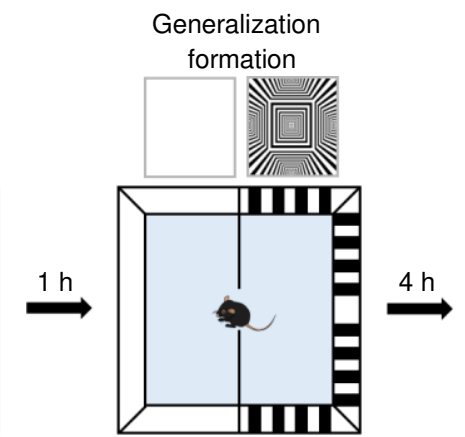

Generalization extinction

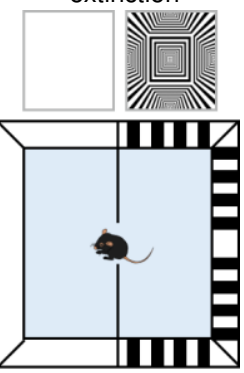

Test phase

Test phase 2

f
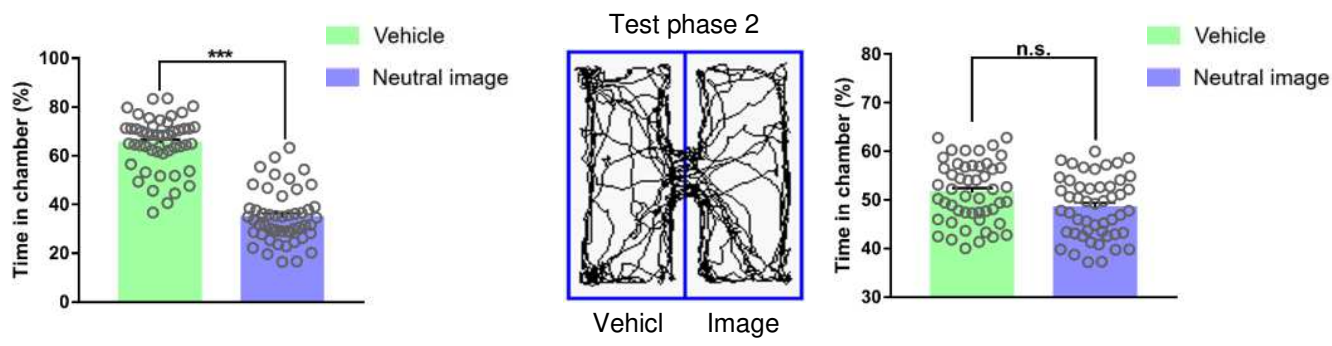


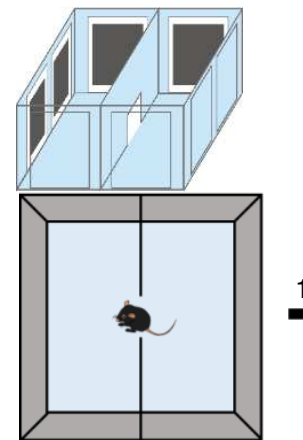

Habituation phase 1

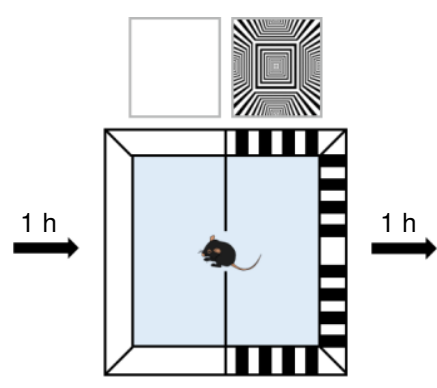

Habituation phase 2

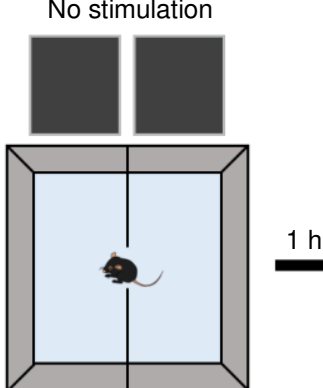

Training phase

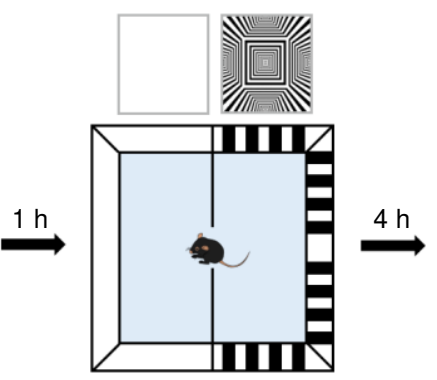

Test phase 1

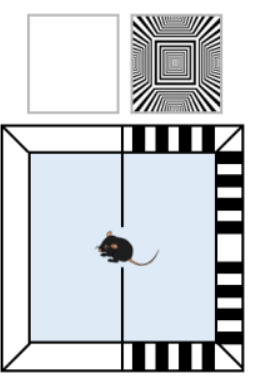

Test phase 2 h

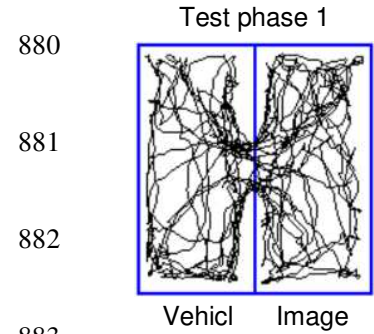

883

884

885

886

887

888

889

890

891

892

893

894

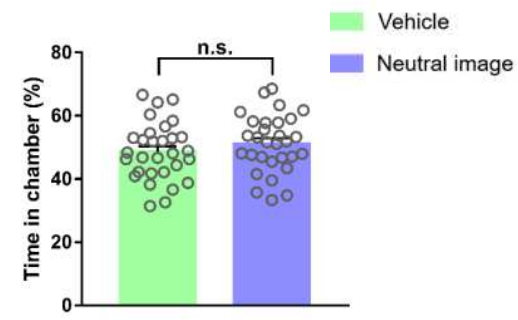

$$
\begin{aligned}
& - \\
& \overline{0} \\
& 0 \\
& \frac{0}{0} \\
& \frac{0}{2} \\
& \frac{1}{90} \\
& \stackrel{5}{-}
\end{aligned}
$$

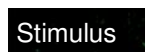

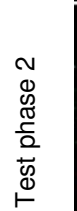
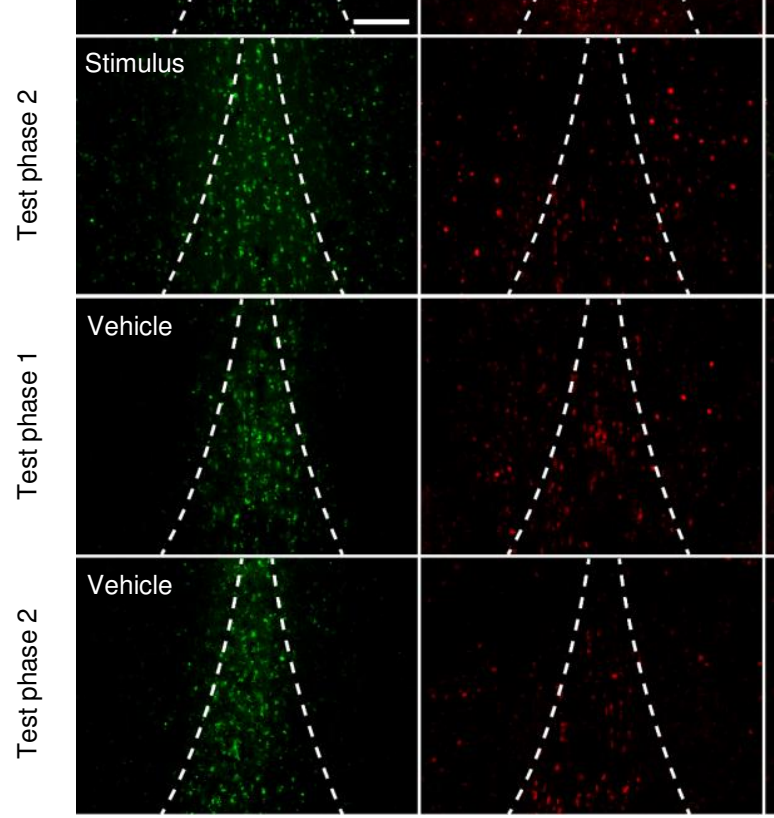
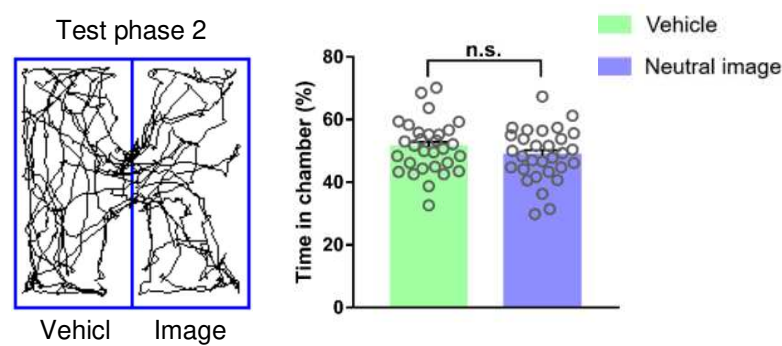

k
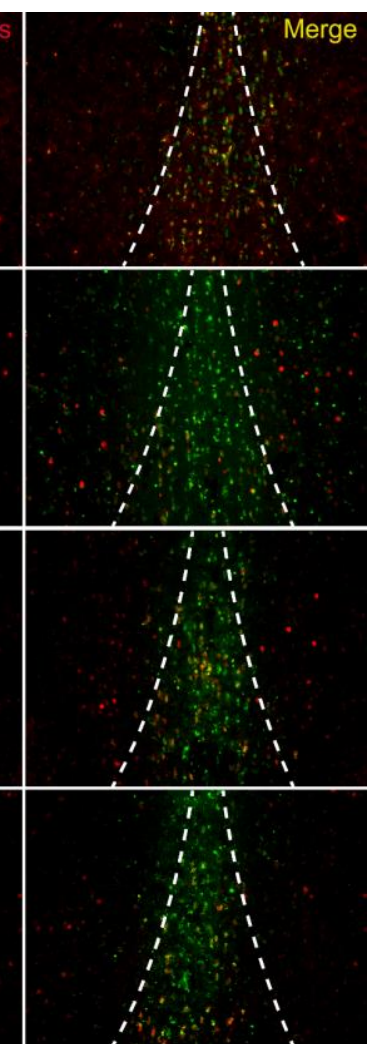

Test phase 1

Test phase 2

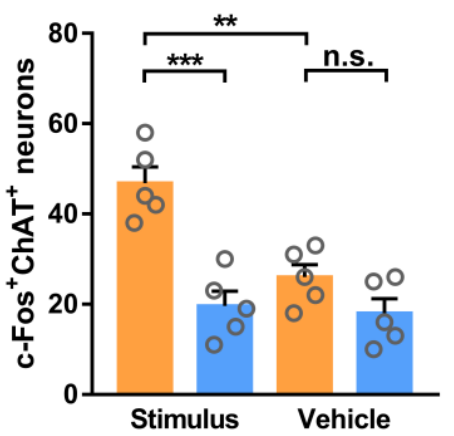


AAV2/9-chat-cre AAV2/9-EF1a-DIO-Gcamp6m
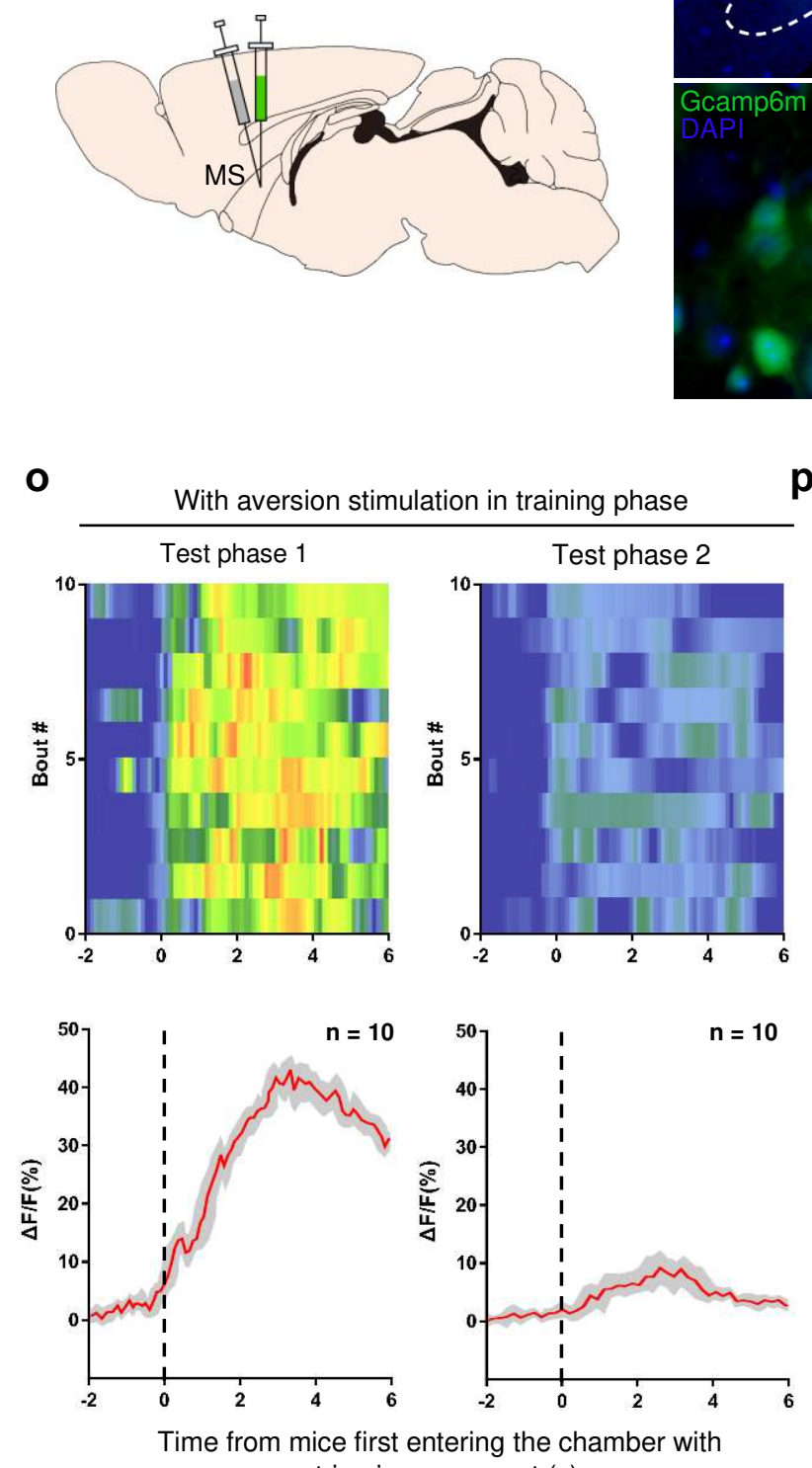
stripe images onset (s)

p

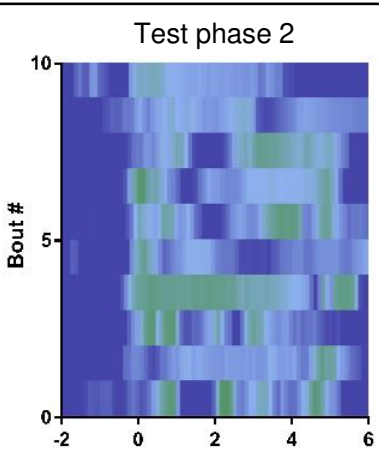

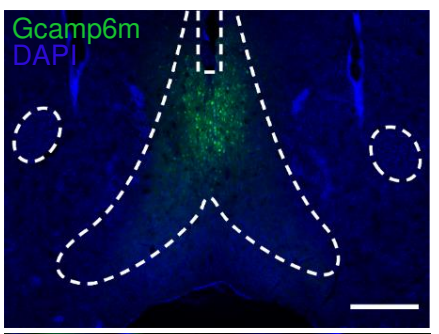

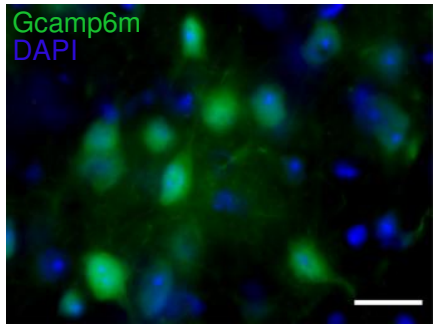

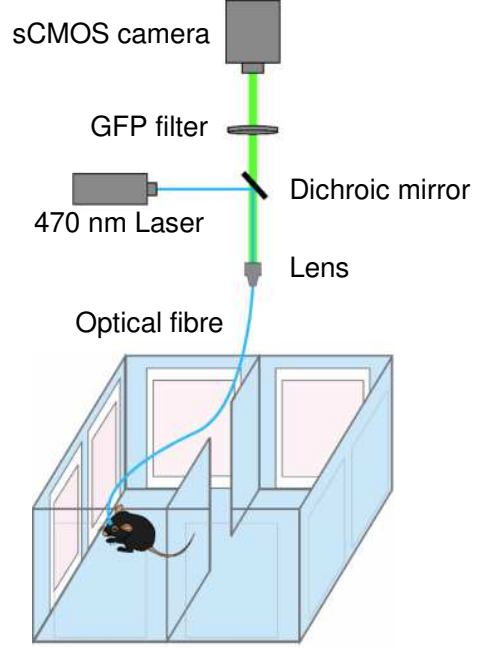
Without stimulation in training phase
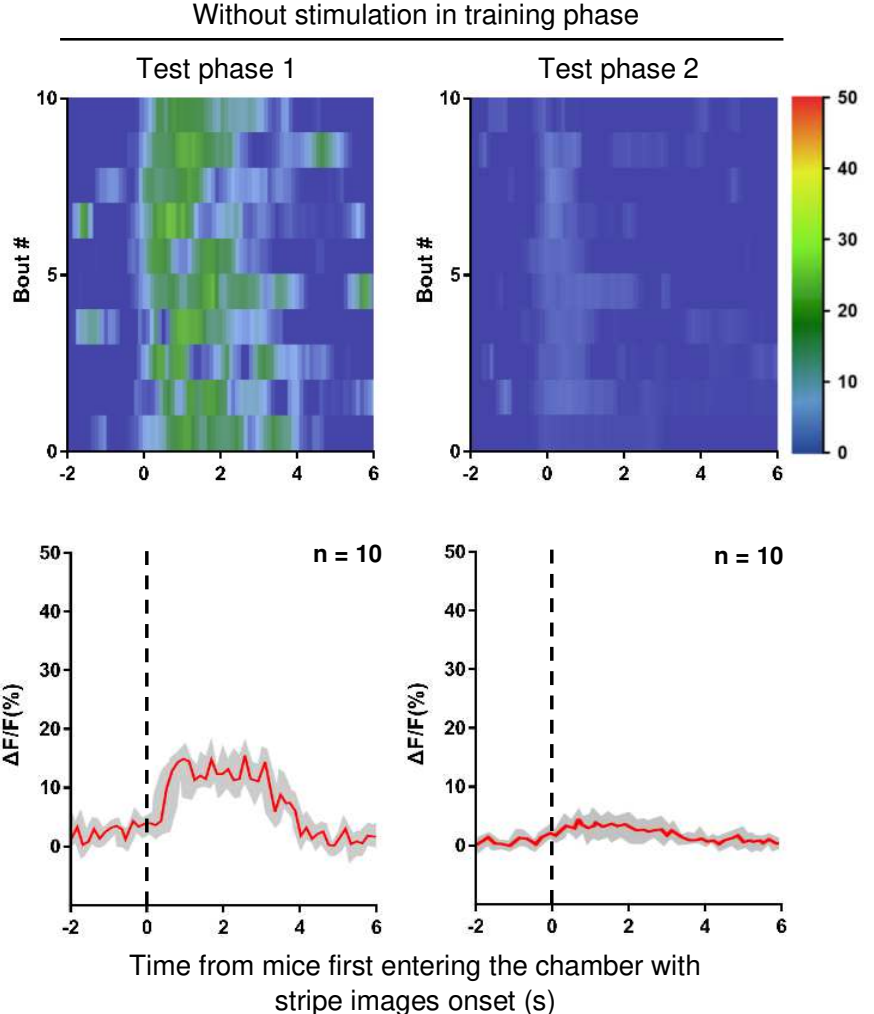
stripe images onset (s)

\section{Fig. 2 MS cholinergic neuron supports rapid generalization formation and extinction of visual}

aversion in mice. a Scheme of a visual aversion task based on real-time place preference (RTPP)

caused by vision. Red arrows show movement direction of the dynamic stripe image. b Representative trace of the locomotion in visual aversion task in mice. c Quantification of the percentage of time spent (c, left) or distance moved (c, right) in each chamber during test phase. $n=15$. d Scheme of a 
generalization task with visual aversion stimulus during training phase. $\mathbf{e}, \mathbf{f}$ Representative movement trace and quantification of the percentage of time spent in each chamber in test phase 1 and 2 (test phase 1: generalization formation phase of visual aversion, $\mathbf{e}$; test phase 2: generalization extinction phase, $\mathbf{f}) . n=50 . \mathbf{g}$ Scheme of a generalization task without visual aversion stimulus during training phase. $\mathbf{h}$, i Representative movement trace and quantification of the percentage of time spent (by mice without suffering aversion stimulus) in each chamber in test phase 1 and $2 . n=30$. $\mathbf{j}$ Representative images of ChAT and c-Fos immunostaining in MS neurons of mice subjected to aversion generalization task. k Quantification of c-Fos ${ }^{+} \mathrm{ChAT}^{+}$neurons in the MS. $n=5$, four sections per mouse. I Schematic diagram of virus injections and optic fiber implantation for recording of GCaMP fluorescence signals of MS cholinergic neurons. $\mathbf{m}$ Representative images of GCaMP6m expression in the MS. $\mathbf{n}$ Schematic diagram of the fiber photometry setup. $\mathrm{Ca}^{2+}$ transients were recorded from GCaMP6m-expressing MS cholinergic neurons in mice subjected to visual aversion generalization task. o, p Upper panel: the heatmap illustration of $\mathrm{Ca}^{2+}$ signals aligned to the onset of aversion generalization formation or extinction assessment. Each row represents one bout. Lower panel: the peri-event plot of the average $\mathrm{Ca}^{2+}$ signal transients. $0 \mathrm{~s}$ means time from mice first entering the chamber with stripe images onset. $n=10$. Thick red lines indicate mean and shaded areas indicate SEM. Mice with aversion stimulus (o), mice without aversion stimulus (p). Scale bar, $200 \mu \mathrm{m}(\mathbf{j}), 400 \mu \mathrm{m}(\mathbf{m}$, top) and $50 \mu \mathrm{m}$ (m, bottom). Data are mean \pm s.e.m. ${ }^{* *} P<0.01,{ }^{* * *} P<0.001$ (two-tailed unpaired Student's $t$ test for $\mathbf{c}, \mathbf{e}, \mathbf{f}, \mathbf{h}$ and i; one-way ANOVA with Tukey's post-hoc analysis for $\mathbf{k}$ ). Exact $p$ values and additional statistical information can be found in Supplementary Table 2. 
945

946

947

948

949

950

951

952

953

954

955

956

957

958

959

960

961

962

963

964

965

966 b

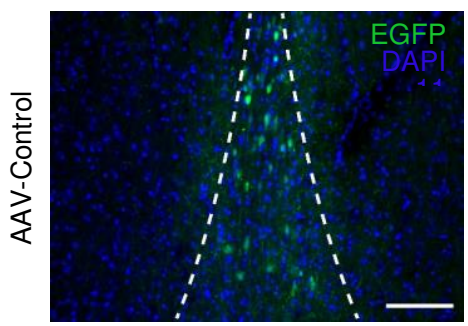

AAV2/9-CAG-DIO-EGFP or

AAV2/9-CAG-DIO-taCaspase3

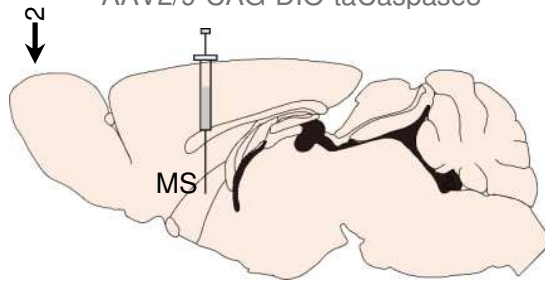

C

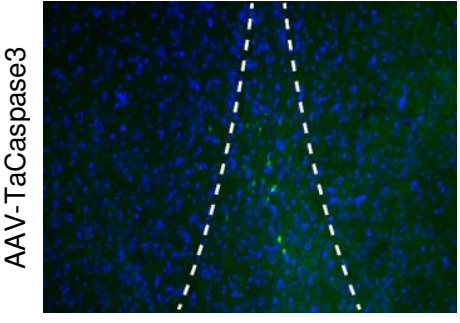

e

Control TaCaspase3

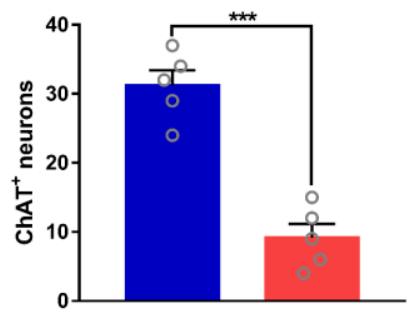

f

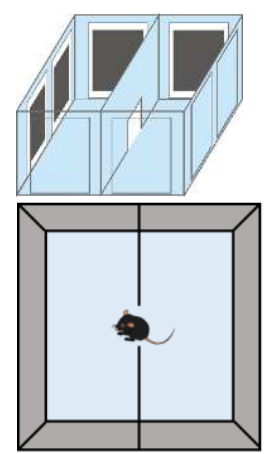

Habituation phase 1

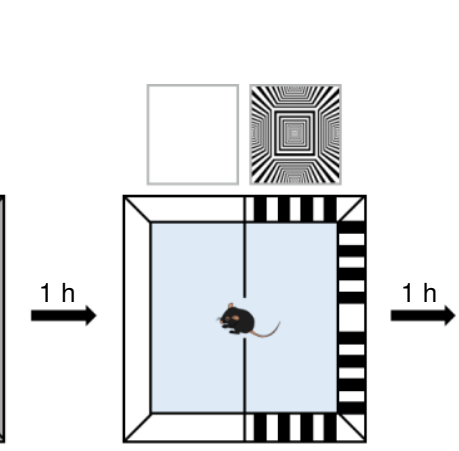

Habituation phase 2

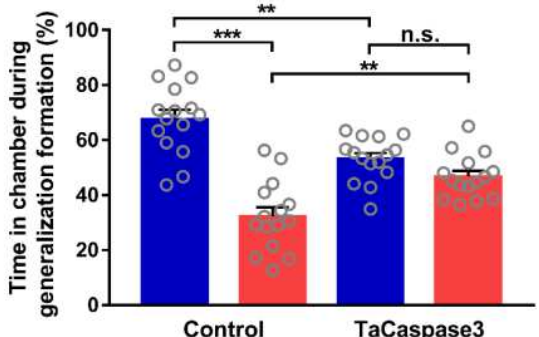

d

AAV-Control
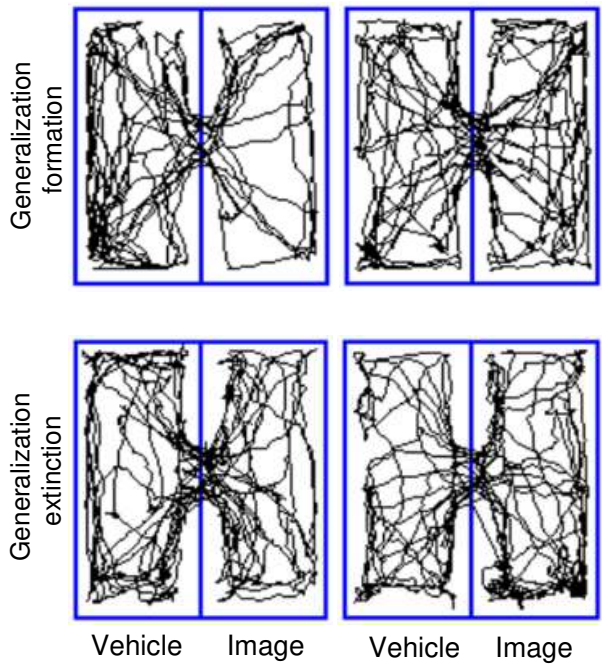

Vehicle $\quad$ Neutral image

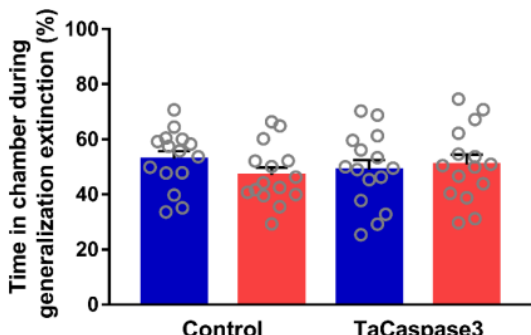

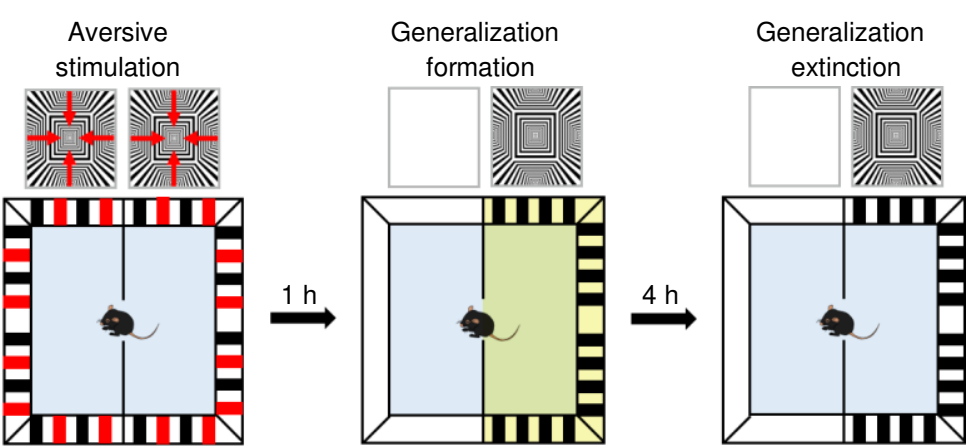

Training phase

Test phase 1

Test phase 2 
AAV2/9-chat-cre-2a-EGFP

969

970

971

972

973

974

975

976

977

978

979

980

981

982

983

984

985

986

987

988

989

990

991

992

993

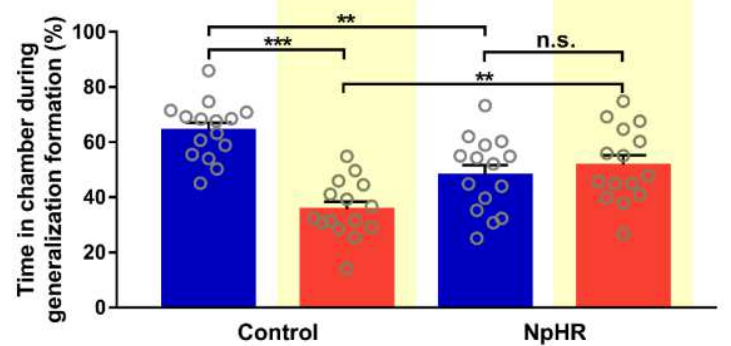

k

AAV2/9-chat-cre-2a-EGFP

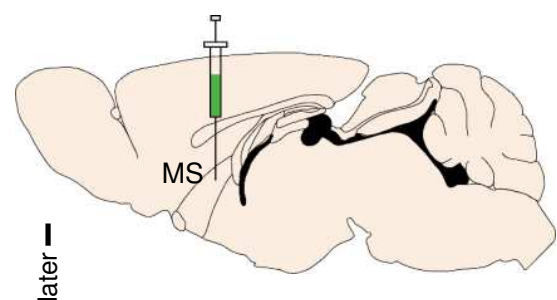

AAV2/9-Ef1 $\alpha$-DIO-mCherry or \ AAV2/9-Ef1a-DIO-hM3D(Gq)-mCherry

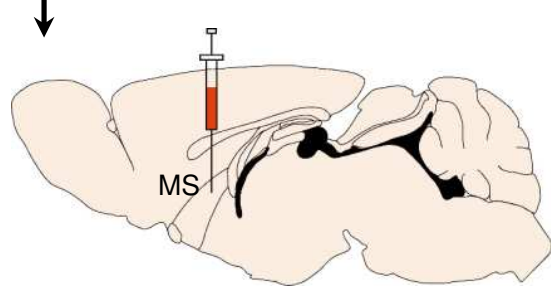

h

Light

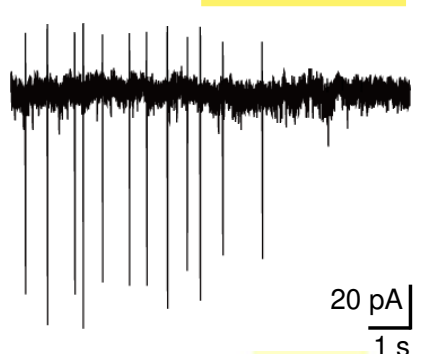

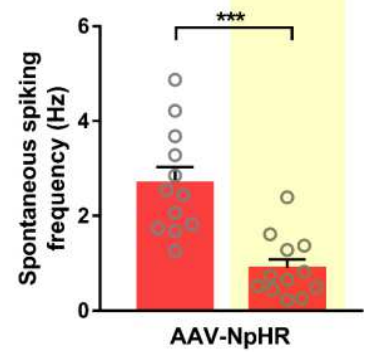

i

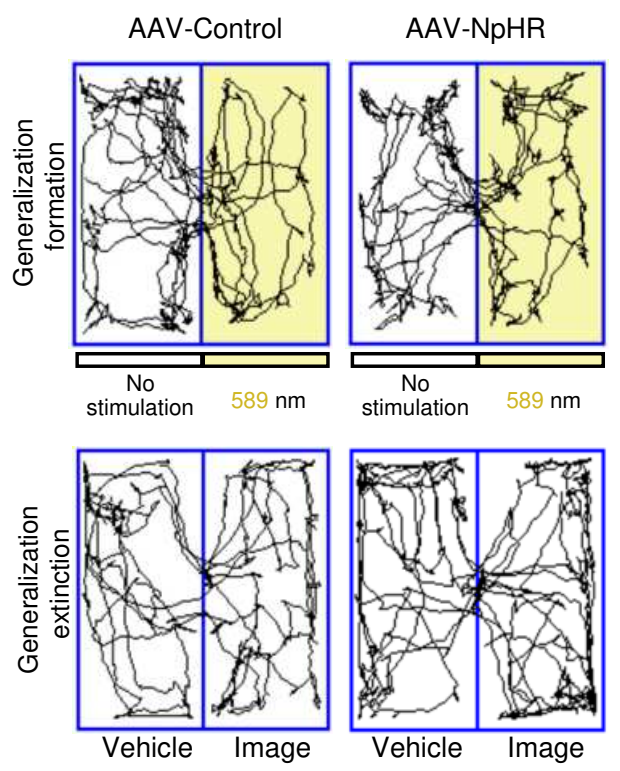

Vehicle $\quad$ Neutral image

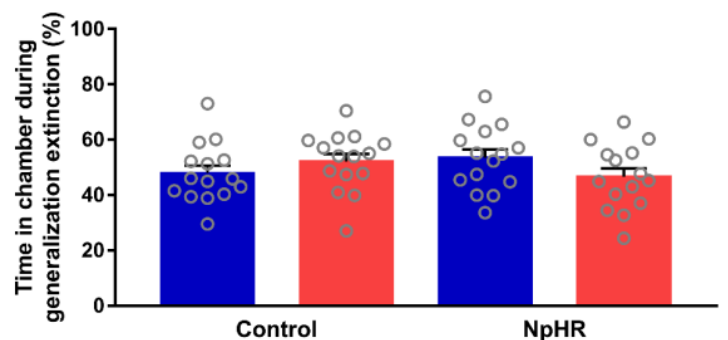

m
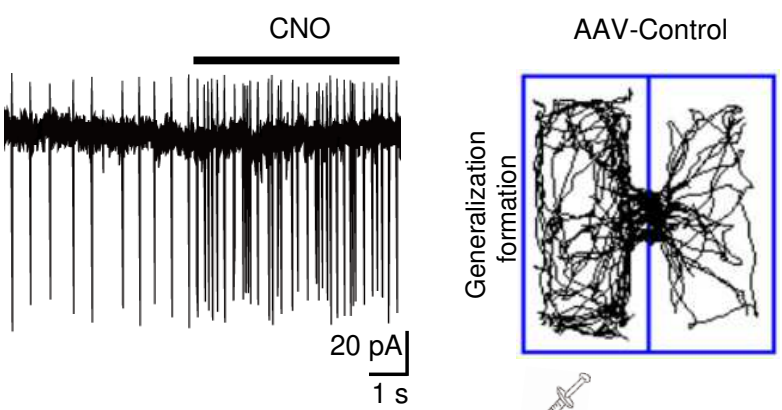

$\operatorname{AAV}-h M 3 D(G q)$
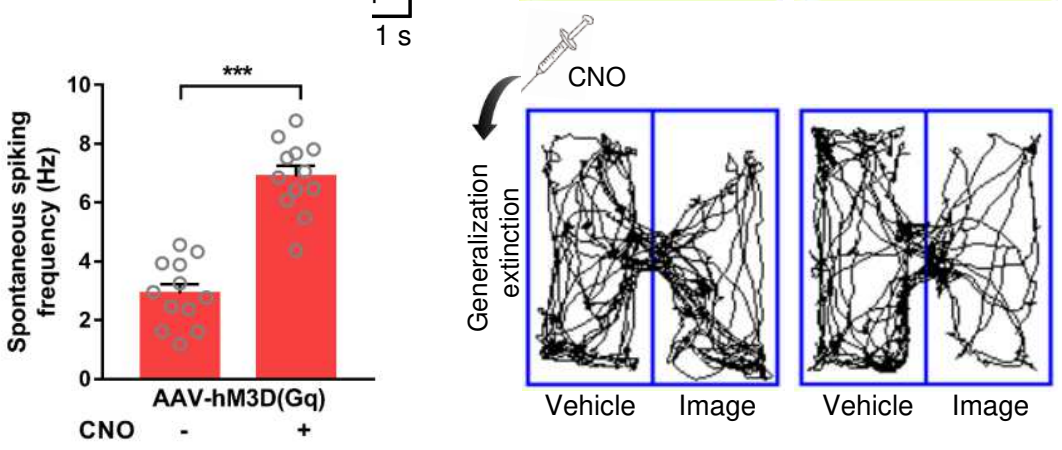


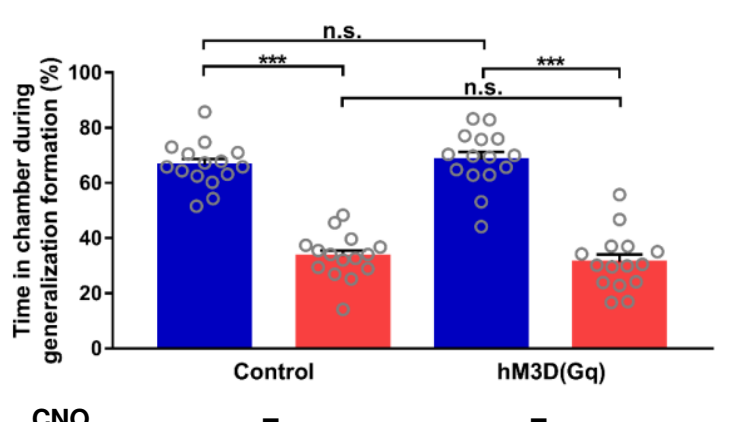

CNO

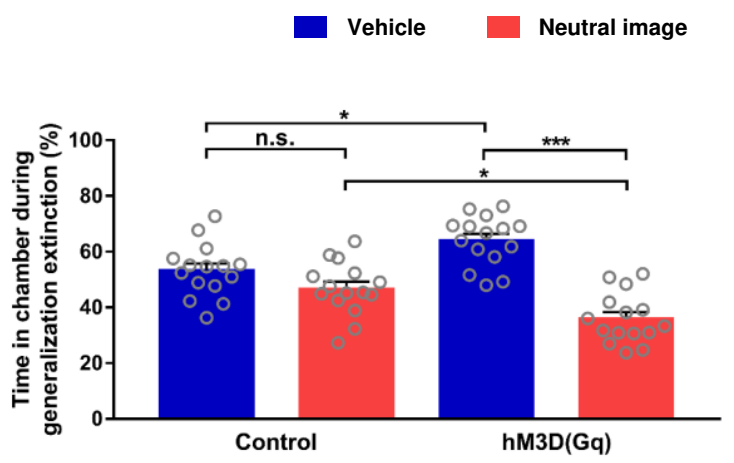

CNO

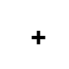

\section{Fig. 3 MS cholinergic neuron activity is necessary for generalization formation and extinction of}

visual aversion. a Schematic diagram depicting virus injections for apoptosis of MS cholinergic neurons. b, c Representative images (b) and quantification (c) of EGFP-labeled MS cholinergic neurons in taCaspase3-expressing or control mice. $n=5$, four sections per mouse. $\mathbf{d}$, e Data of visual aversion generalization task in mice whose MS cholinergic neurons were inhibited with apoptosis manipulation (representative trace of mice, $\mathbf{d}$; quantification of the percentage of time spent in each chamber during generalization formation and extinction phases, e). $n=15$. $\mathbf{f}$ Scheme of visual aversion generalization task with optogenetic inhibition approach using a yellow-light $(589 \mathrm{~nm}, 10 \mathrm{~mW}) . \mathbf{g}$ Schematic diagram depicting virus injections for optogenetic inhibition of MS cholinergic neurons. $\mathbf{h}$ Yellow light decreased spontaneous firing in MS cholinergic neurons expressing NpHR-mCherry in brain slices. $n=12$ cells from four mice. $\mathbf{i}, \mathbf{j}$ Data of visual aversion generalization task in mice whose MS cholinergic neurons were inhibited with optogenetic manipulation (representative trace of mice, $\mathbf{i}$; quantification of the percentage of time spent in each chamber during generalization phases, $\mathbf{j}$ ). $n=15$. $\mathbf{k}$ Schematic diagram depicting virus injections for chemogenetic activation of MS cholinergic neurons. I Bath application of CNO $(5 \mu \mathrm{M})$ increased spontaneous firing in MS cholinergic neurons expressing hM3Dq-mCherry in brain slices. $n=12$ cells from four mice. m, $\mathbf{n}$ Data of visual aversion 
1019 generalization task in mice whose MS cholinergic neurons were activated with chemogenetic 1020 manipulation (representative trace of mice, $\mathbf{m}$; quantification of the percentage of time spent in each 1021 chamber during generalization phases, $\mathbf{n})$. Mice were intraperitoneally injected with CNO (2.5 mg/kg) 1022 after generalization formation test. $n=15$. Scale bar, $200 \mu \mathrm{m}(\mathbf{b})$. Data are mean \pm s.e.m. $* P<0.05, * * P$ $1023<0.01, * * * P<0.001$, n.s.: $p>0.05$ (two-tailed unpaired Student's $t$ test for $\mathbf{c}, \mathbf{h}$ and $\mathbf{l}$; one-way ANOVA 1024 with Tukey's post-hoc analysis for $\mathbf{e}, \mathbf{j}$ and $\mathbf{n}$ ). Exact $p$ values and additional statistical information can 1025 be found in Supplementary Table 2. 


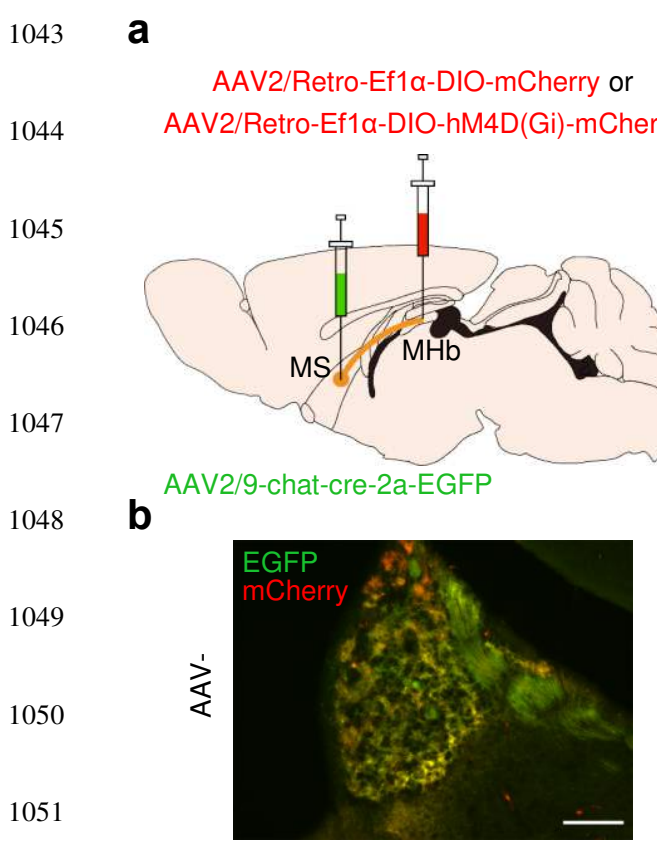

C

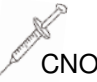

d

Vehicle Neutral image
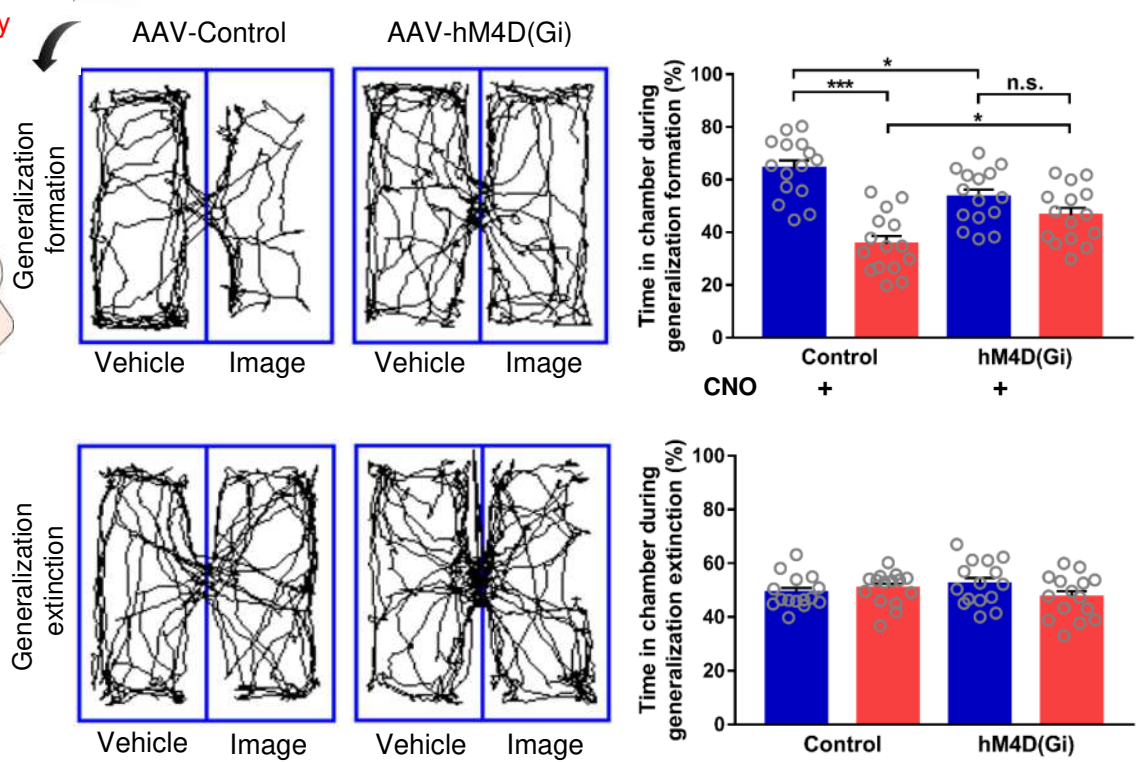

1052

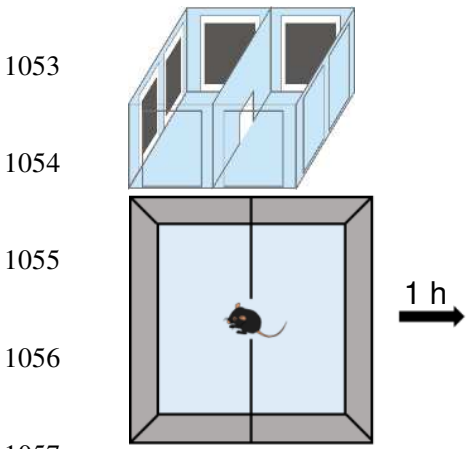

1057

Habituation phase 1
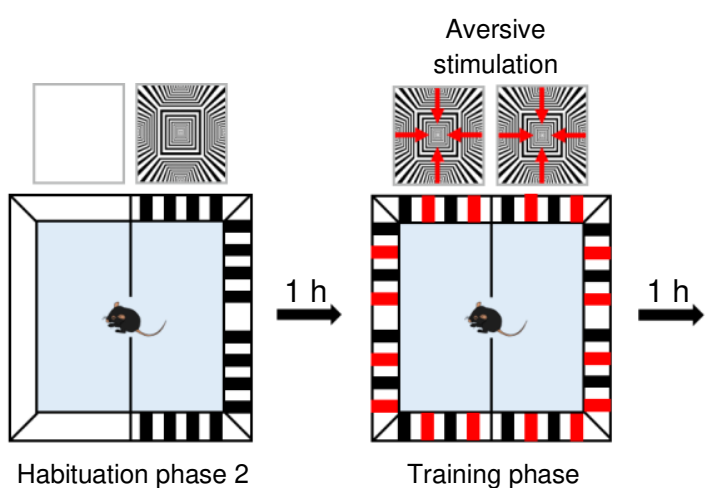

Training phase

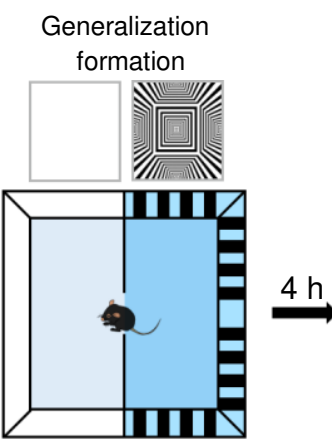

Test phase 1
Generalization extinction

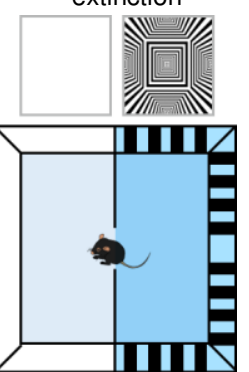

Test phase 2
$1058 \quad \mathbf{f}$

1059

1060

1061

1062

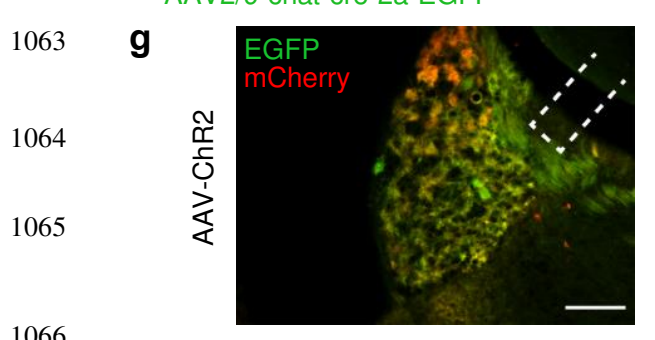

h
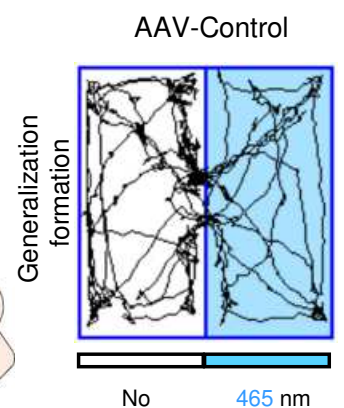

stimulation

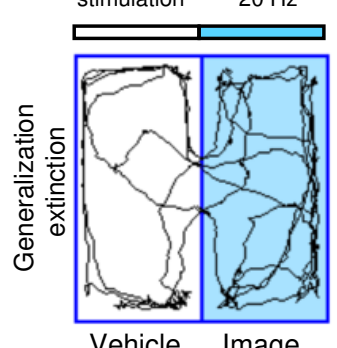

AAV-ChR2

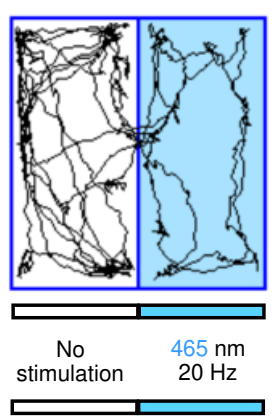

i

Vehicle Neutral image
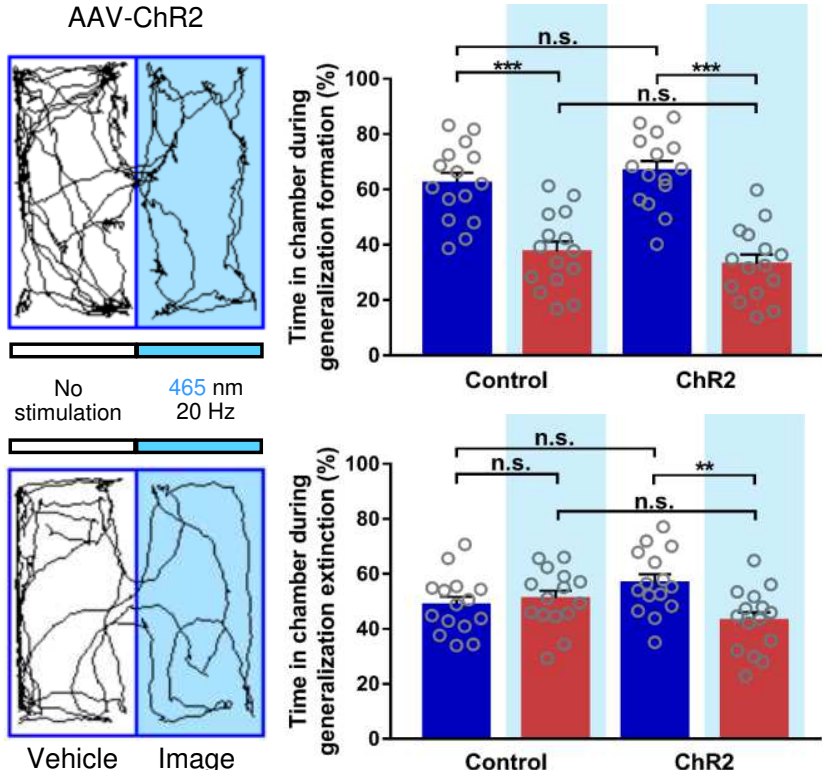
Fig. $4 \mathrm{MS} \rightarrow \mathrm{MHb}$ cholinergic circuit drives generalization formation and extinction of visual

aversion. a Schematic diagram depicting virus injections for chemogenetic inhibition of $\mathrm{MS} \rightarrow \mathrm{MHb}$ cholinergic circuits. b Representative image of AAVs expression in the MHb three weeks after AAV$\mathrm{hM} 4 \mathrm{D}(\mathrm{Gi})$ injection. c, d Data of visual aversion generalization task in mice whose $\mathrm{MS} \rightarrow \mathrm{MHb}$ cholinergic circuits were inhibited with chemogenetic manipulation (representative trace of mice, $\mathbf{c}$; quantification of the percentage of time spent in each chamber during generalization formation and extinction phases, d). Mice were intraperitoneally injected with CNO $(2.5 \mathrm{mg} / \mathrm{kg}) 30 \mathrm{~min}$ before generalization formation test. $n=15$. e Scheme of visual aversion generalization task with an optogenetic activation approach using blue-light pulse (465 nm, $20 \mathrm{~Hz}, 40 \mathrm{~ms})$. f Schematic diagram depicting virus injections for optogenetic activation of $\mathrm{MS} \rightarrow \mathrm{MHb}$ cholinergic circuits. $\mathbf{g}$ Representative image of AAVs expression in the MHb three weeks after AAV-ChR2 injection. $\mathbf{h}, \mathbf{i}$ Data of visual aversion generalization task in mice whose $\mathrm{MS} \rightarrow \mathrm{MHb}$ cholinergic circuits were activation with optogenetic manipulation (representative trace of mice, $\mathbf{h}$; quantification of the percentage of time spent in each chamber during generalization formation and extinction phases, i). Scale bar, $100 \mu \mathrm{m}$ (b and g). Data are mean \pm s.e.m. $* P<0.05,{ }^{* *} P<0.01,{ }^{* * *} P<0.001$, n.s.: $p>0.05$ (one-way ANOVA with Tukey's post-hoc analysis for $\mathbf{d}$ and i). Exact $p$ values and additional statistical information can be found in Supplementary Table 2 . 
e

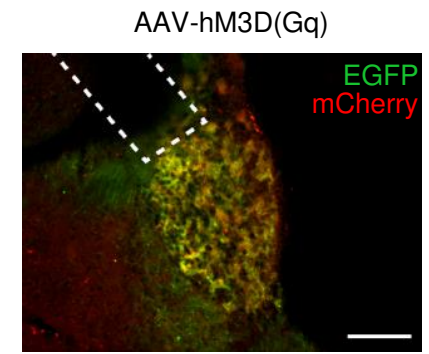
(CNO+Pirenzepine)

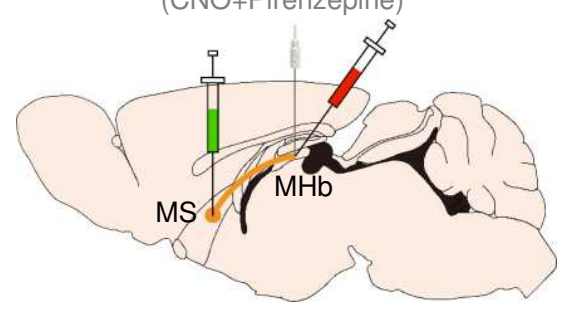

AAV2/9-chat-cre-2a-EGFP AAV2/Retro-Ef1a-DIO-hM3D(Gq)-mCherry (CNO) or
AAV2/Retro-Ef1a-DIO-hM3D(Gq)-mCherry

d
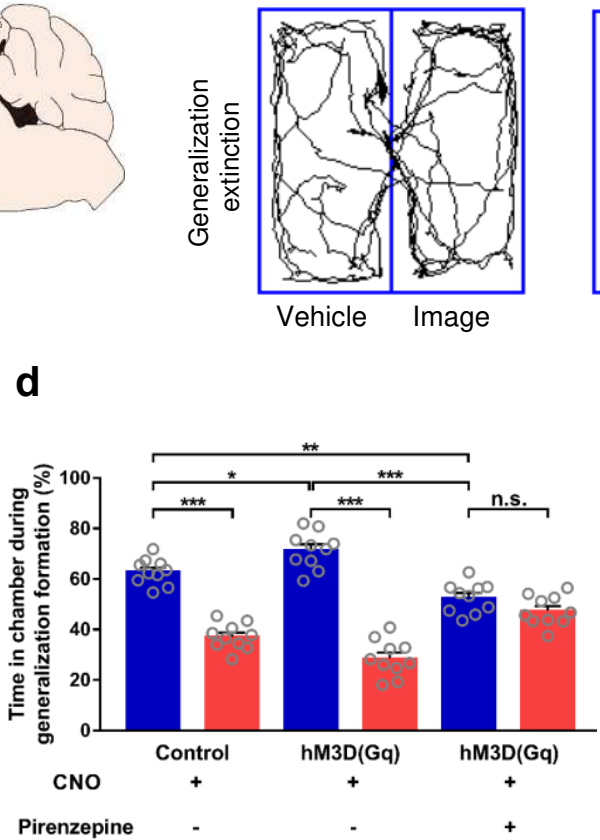

g AAV2/9-CaMKIla-cre AAV2/9-Ef1a-DIO-ACh2.0

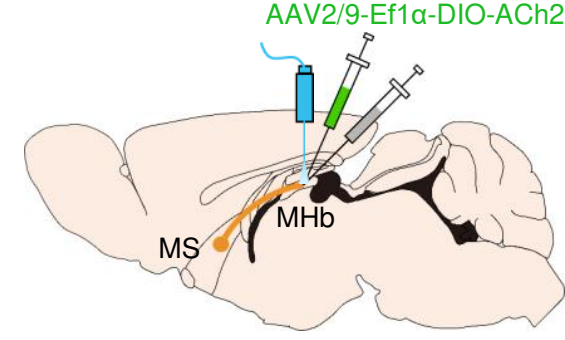

\section{f}
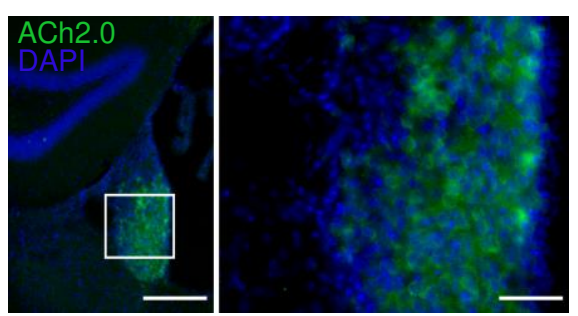

Generalization
C

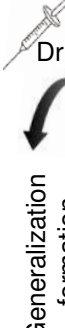

AAV-Control (CNO) (CNO)
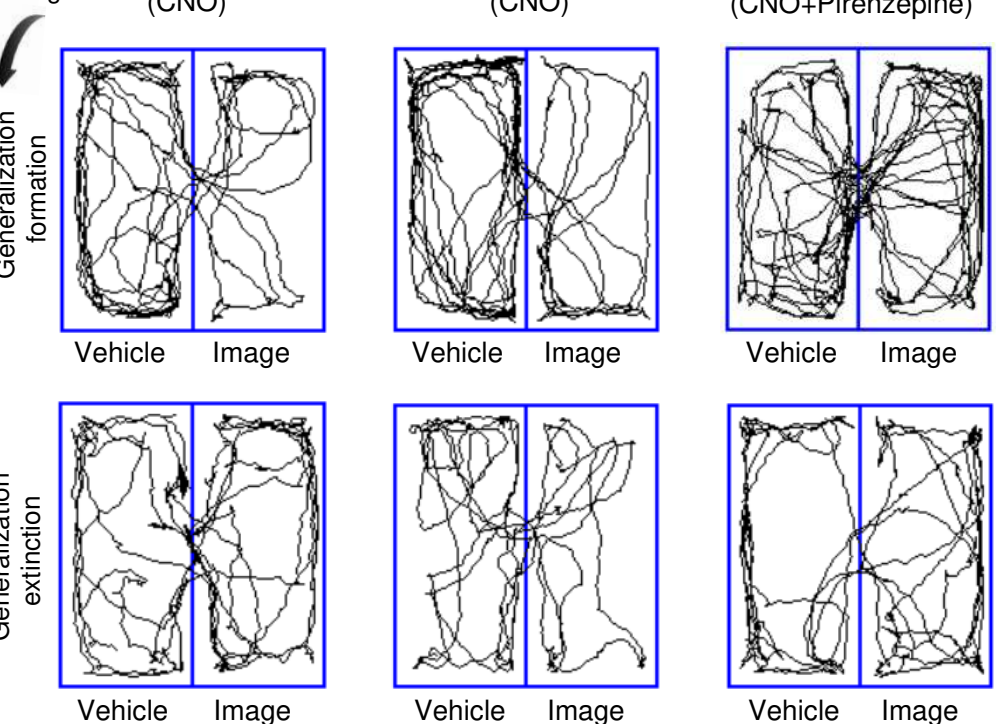

Vehicle Image

Vehicle

Neutral image
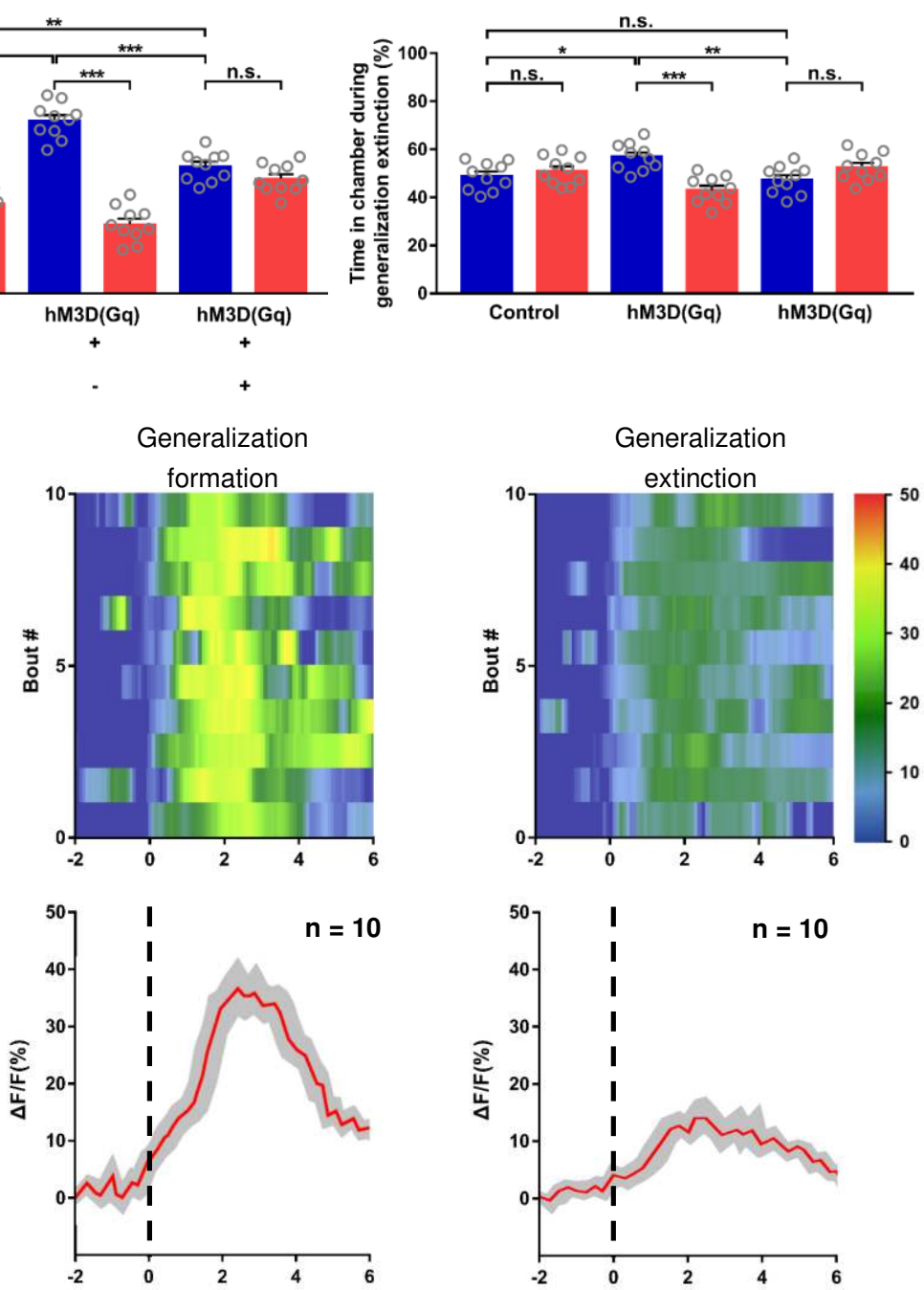

Time from mice first entering the chamber with stripe images onset (s) 
Fig. 5 M1 mAChRs and glutamatergic neurons in the MHb are involved in generalization

formation and extinction of visual aversion. a Schematic diagram depicting virus and drug injections for chemogenetic activation of $\mathrm{MS} \rightarrow \mathrm{MHb}$ cholinergic circuits and blockade of MHb M1 mAChRs. b Representative image of AAVs expression in the $\mathrm{MHb}$ three weeks after AAV-hM3D(Gq) injection. c, d Data of visual aversion generalization task in mice whose $\mathrm{MS} \rightarrow \mathrm{MHb}$ cholinergic circuits were activated with chemogenetic manipulation while $\mathrm{MHb}$ M1 mAChRs were blocked by pirenzepine (representative trace of mice, $\mathbf{c}$; quantification of the percentage of time spent in each chamber during generalization formation and extinction phases, d). Mice were injected with $\mathrm{CNO}(3.0 \mu \mathrm{M} / 0.1 \mu \mathrm{L} /$ side $)$ or $\mathrm{CNO}+$ Pirenzepine $(1.5 \mu \mathrm{g} / 0.1 \mu \mathrm{L} /$ side $)$ into the $\mathrm{MHb}$ by cannulas $30 \mathrm{~min}$ before generalization formation test. $n=10$. e Schematic diagram of virus injections and optic fiber implantation for recording of acetylcholine fluorescence signals of $\mathrm{MHb}$ glutamatergic neurons. $\mathbf{f}$ Representative images of ACh2.0 expression in the MHb three weeks after AAV-ACh2.0 injection. g Acetylcholine transmissions in $\mathrm{MHb}$ glutamatergic neurons were involved in generalization formation (g, left) and extinction (g, right) of visual aversion. Upper panel, the heatmap illustration of acetylcholine signals aligned to the onset of individual generalization assessments. Each row represents one bout, and a total of 10 bouts are illustrated. Lower panel, the peri-event plot of the average acetylcholine signal transients. $0 \mathrm{~s}$ means time from mice first entering the chamber with stripe images onset. $n=10$. Thick red lines indicate mean and shaded areas indicate SEM. Scale bar, $100 \mu \mathrm{m}(\mathbf{b}), 200 \mu \mathrm{m}(\mathbf{f}$, left) and 50 $\mu \mathrm{m}$ (f, right). Data are mean \pm s.e.m. ${ }^{*} P<0.05,{ }^{*} P<0.01,{ }^{* * *} P<0.001$, n.s.: $p>0.05$ (one-way ANOVA with Tukey's post-hoc analysis for d). Exact $p$ values and additional statistical information can be found in Supplementary Table 2. 
Figure. 6

a

AAV2/Retro-Ef1a-DIO-mCherry +AAV2/9-CaMKIla-mCherry (CNO)

or AAV2/Retro-Ef1a-DIO-hM3D(Gq)-mCherry + AAV2/9-CaMKIla-mCherry (CNO)

or

AAV2/Retro-Ef1a-DIO-hM3D(Gq)-mCherry +AAV2/9-CaMKIla-hM4D(Gi)-mCherry (CNO)

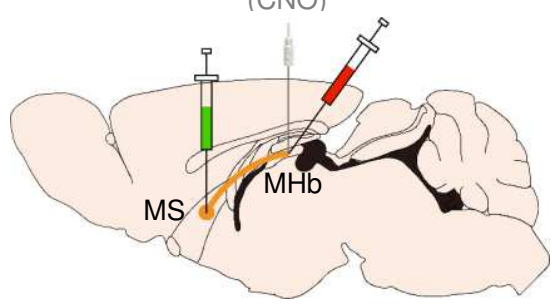

AAV2/9-chat-cre-2a-EGFP
C

CNO

AAV/R-DIO-mCherry AAV/R-DIO-hM3D $(\mathrm{Gq}) \quad$ AAV/R-DIO-hM3D $(\mathrm{Gq}$ AAV/9-CaMKIla-mCherry AAV/9-CaMKIla-mCherry AAV/9-CaMKIla-hM4D(Gi)
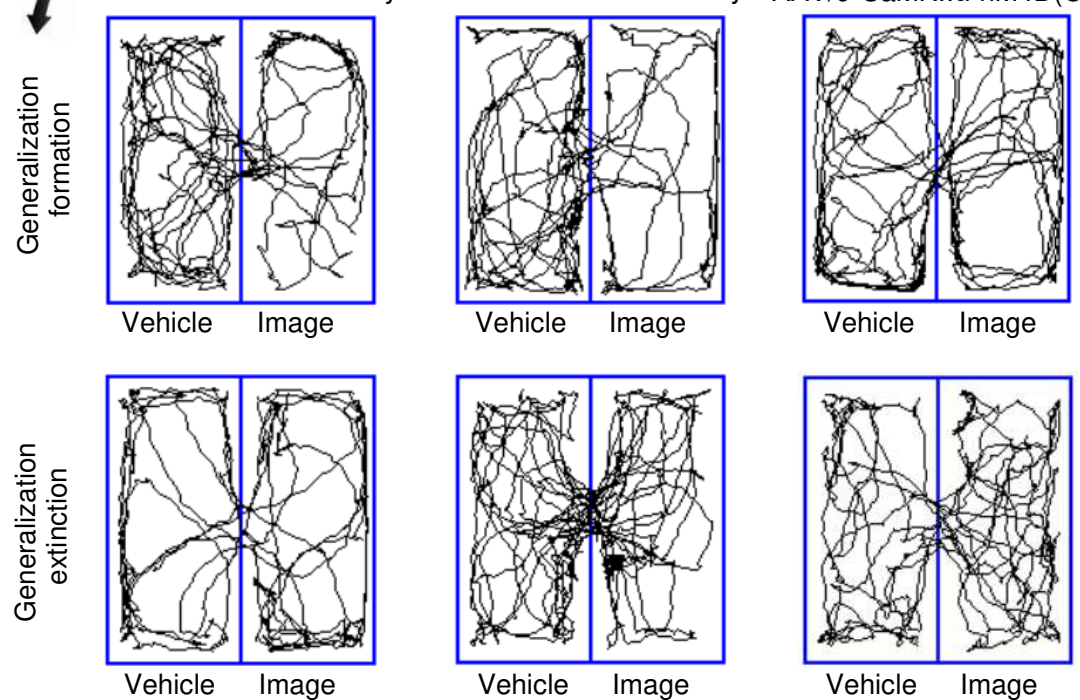

Vehicle Image b AAV-CaMKIla-hM4D(Gi) d

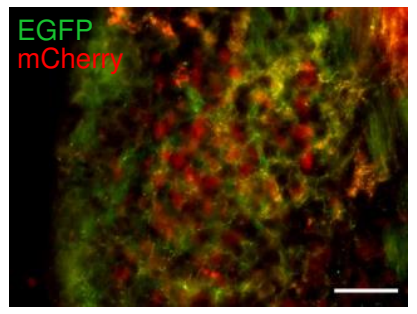

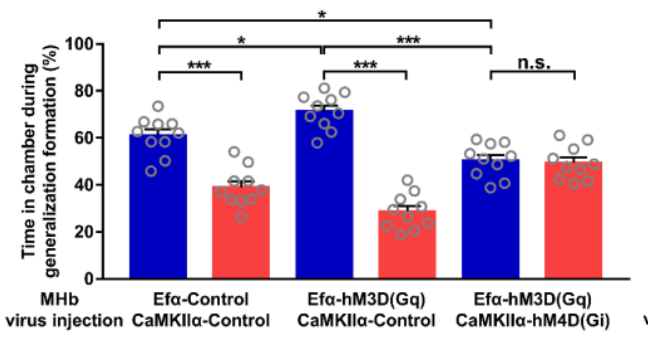
CNO + e

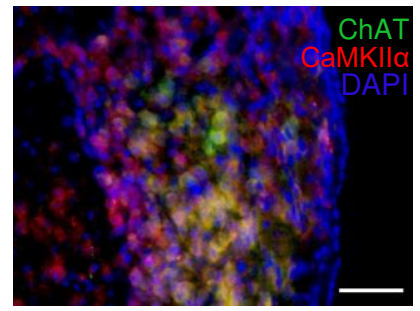

f

AAV2/9-chat-cre

+ AAV2/9-Ef1 $\alpha$-DIO-hM3D(Gq)-mCherry

$$
\text { (ACSF) }
$$

or

AAV2/9-chat-cre

+ AAV2/9-Ef1a-DIO-mCherry (CNO)

or

AAV2/9-chat-cre

+ AAV2/9-Ef1 $\alpha$-DIO-hM3D(Gq)-mCherry (CNO)

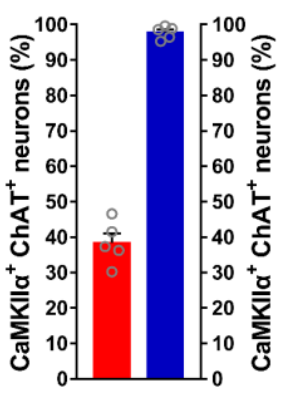

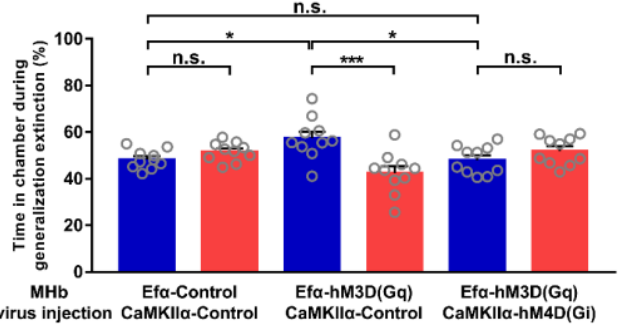

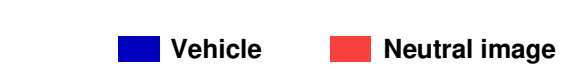


$1165 \quad \mathbf{h}$

1166

1167

1168

1169

1170

1171

1172

1173

1174

1175

1176

1177

1178

1179

1180

1181

1182

1183

1184

1185
$\mathbf{h}_{\mathrm{CNO}}$
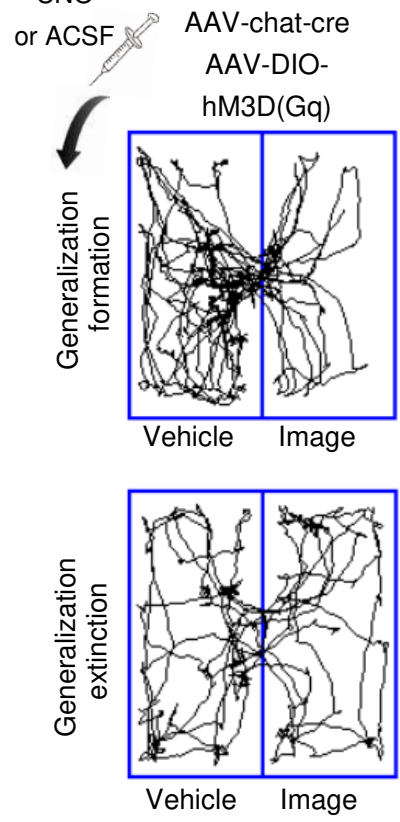

AAV-chat-cre AAV-DIO-mCherry (CNO)
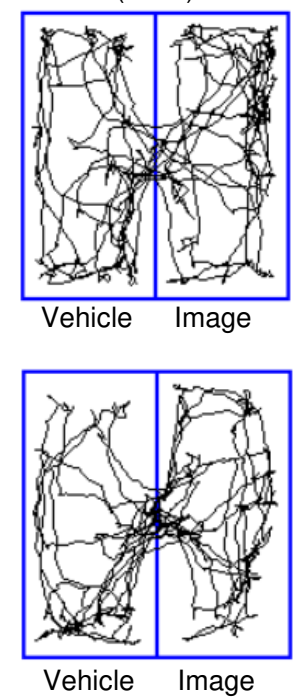

AAV-chat-cre AAV-DIO-hM3D(Gq)
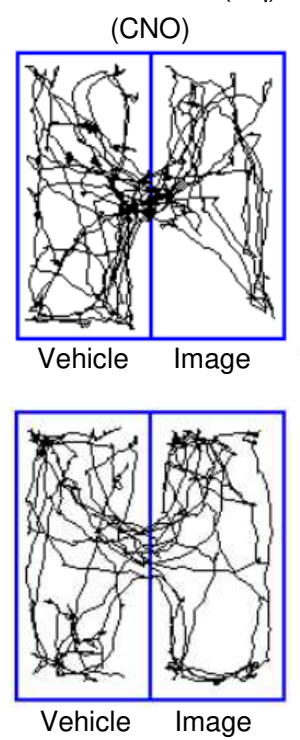

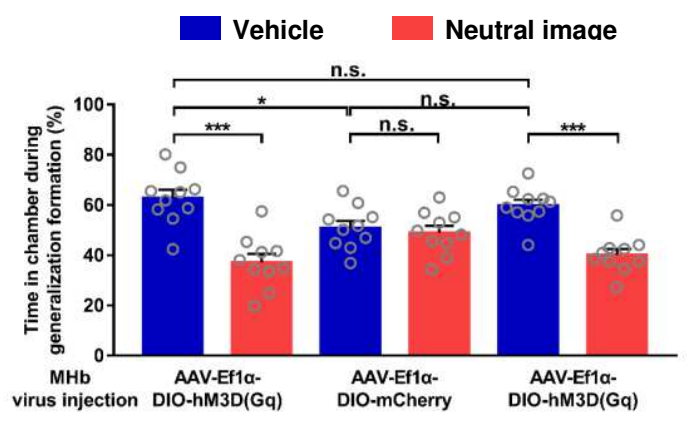

CNO

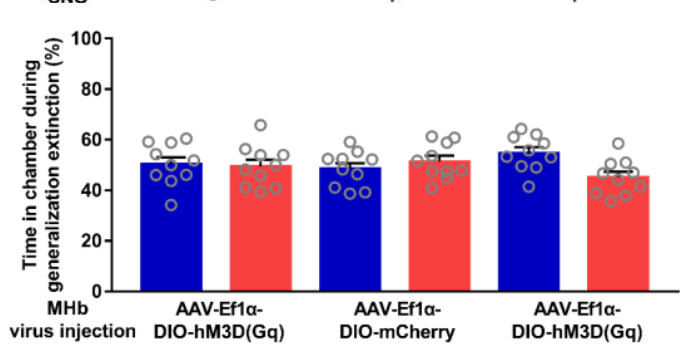

Fig. $6 \mathrm{MHb}$ glutamatergic/cholinergic neurons participate in generalization formation and

extinction of visual aversion. a Schematic diagram depicting virus and drug injections for activation

of $\mathrm{MS} \rightarrow \mathrm{MHb}$ cholinergic circuits and inhibition of $\mathrm{MHb}$ glutamatergic neurons with chemogenetic approaches. b Representative image of expressions of AAVs (used in a) in the MHb. c, d Data of visual aversion generalization task in mice whose $\mathrm{MS} \rightarrow \mathrm{MHb}$ cholinergic circuits were activated while $\mathrm{MHb}$ glutamatergic neurons were inhibited with chemogenetic manipulations (representative trace of mice, c; quantification of the percentage of time spent in each chamber during generalization formation and extinction phases, d). $\mathrm{CNO}(3.0 \mu \mathrm{M} / 0.1 \mu \mathrm{L} /$ side $)$ was bilaterally injected into the $\mathrm{MHb}$ by cannulas 30 min before generalization formation test. $n=10$. e Representative image (e, top) and quantification (e, bottom) of co-localization of CaMKIIa (red) with ChAT (green) in MHb neurons by immunohistochemistry. $n=5$, four sections per mouse. f Schematic diagram depicting virus and drug injections for chemogenetic inhibition of $\mathrm{MS} \rightarrow \mathrm{MHb}$ cholinergic circuits and activation of $\mathrm{MHb}$ glutamatergic/cholinergic neurons with chemogenetic approaches. g Representative image of expressions of AAVs (used in $\mathbf{f}$ ) in the MHb. $\mathbf{h}, \mathbf{i}$ Data of visual aversion generalization task in mice 


7

198

whose $\mathrm{MS} \rightarrow \mathrm{MHb}$ cholinergic circuits were inhibited while MHb glutamatergic/cholinergic neurons were activated with chemogenetic manipulations (representative trace of mice, $\mathbf{h}$; quantification of the percentage of time spent in each chamber during generalization formation and extinction phases, i). CNO $(3.0 \mu \mathrm{M} / 0.1 \mu \mathrm{L} /$ side) was bilaterally injected into the $\mathrm{MHb}$ by cannulas 30 min before generalization formation test. $n=10$. Scale bar, $50 \mu \mathrm{m}(\mathbf{b}, \mathbf{e}$ and $\mathbf{g})$. Data are mean \pm s.e.m. $* P<0.05$, $* * * P<0.001$, n.s.: $P>0.05$ (one-way ANOVA with Tukey's post-hoc analysis for $\mathbf{d}$ and $\mathbf{i}$ ). Exact $p$ values and additional statistical information can be found in Supplementary Table 2. 
Figure. 7

a

AAV2/9-chat-EGFP

+ AAV2/9-CaMKIla-mCherry

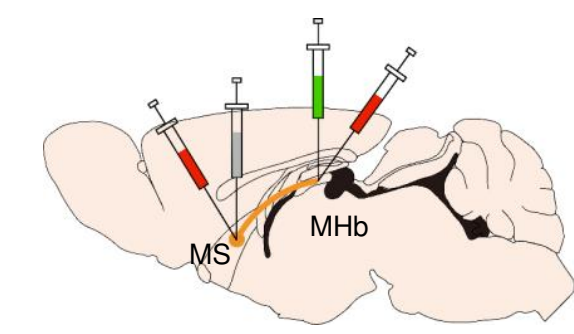

AAV2/9-chat-cre

+ AAV2/9-Ef1a-DIO-ChR2-mCherry

d

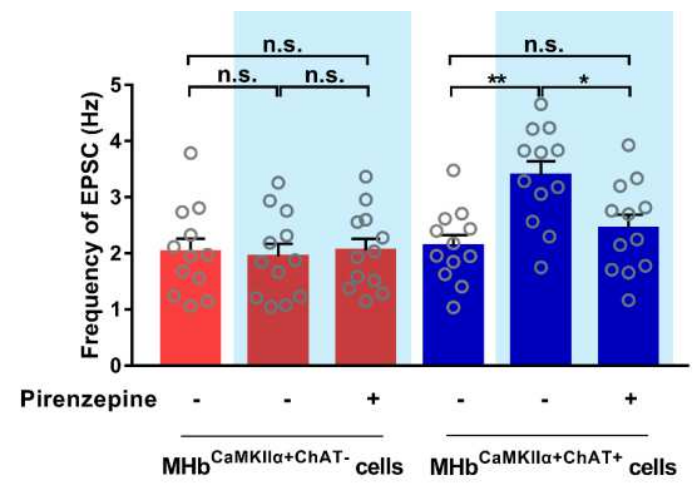

b

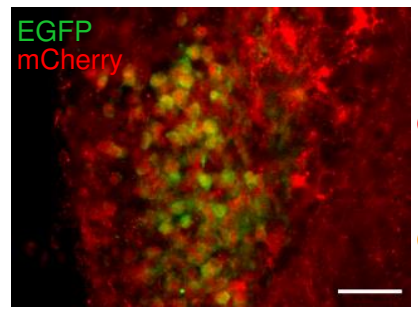

$25 \mathrm{pA}$

C

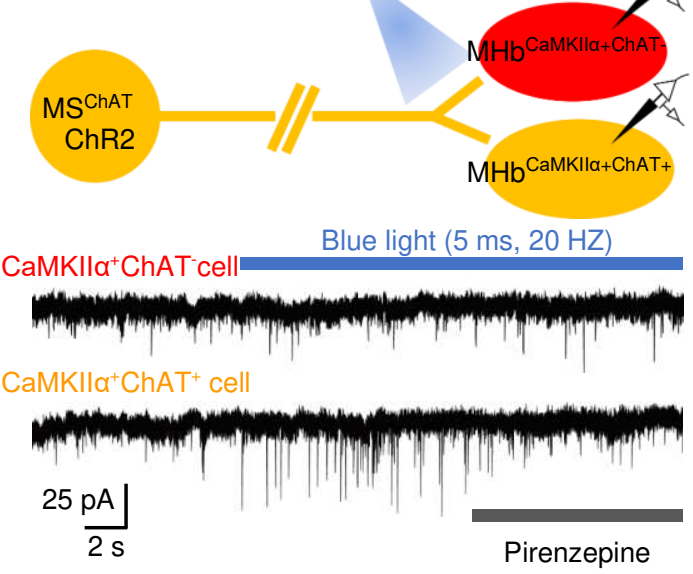

e

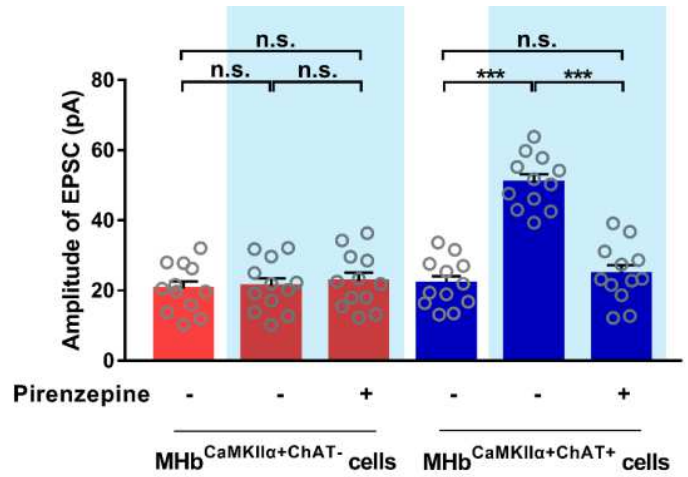

Fig. 7 MHb glutamatergic/cholinergic neurons mediate regulatory effects of $\mathrm{MS} \rightarrow \mathrm{MHb}$

cholinergic circuit via M1 mAChRs. a Schematic diagram depicting virus injections for whole-cell recording of EPSC in MHb neurons. b Representative image of AAVs expression in the MHb three weeks after AAV-ChR2 injection. MHb glutamatergic/cholinergic neurons $\left(\mathrm{MHb}^{\mathrm{CaMKII} \alpha+\mathrm{ChAT}+}\right)$ were labeled with chat-EGFP and CaMKII $\alpha-m C h e r r y . ~ M H b$ glutamatergic neurons without releasing acetylcholine $\left(\mathrm{MHb}^{\mathrm{CaMKII} \alpha+\mathrm{ChAT}-}\right)$ were labeled with CaMKII $\alpha$-mCherry alone. Scale bar, $50 \mu \mathrm{m}$. c Representative traces of EPSCs in $\mathrm{MHb}^{\mathrm{CaMKII}+\mathrm{ChAT}+}$ and $\mathrm{MHb}^{\mathrm{CaMKII}+\mathrm{ChAT}-}$ neurons. EPSCs were recorded at $-70 \mathrm{mV}$. Optogenetic stimulations $(465 \mathrm{~nm}, 5 \mathrm{~ms}, 20 \mathrm{~Hz})$ of ChR2 ${ }^{+}$axonal terminals and M1 mAChRs blockade were performed during the recording of EPSCs. d, e Quantification of frequency $(\mathbf{m})$ and amplitude (n) of EPSCs. $n=12$ cells from four mice. Data are mean \pm s.e.m. $* P<$ 

and $\mathbf{n})$. Exact $p$ values and additional statistical information can be found in Supplementary Table 2 . 

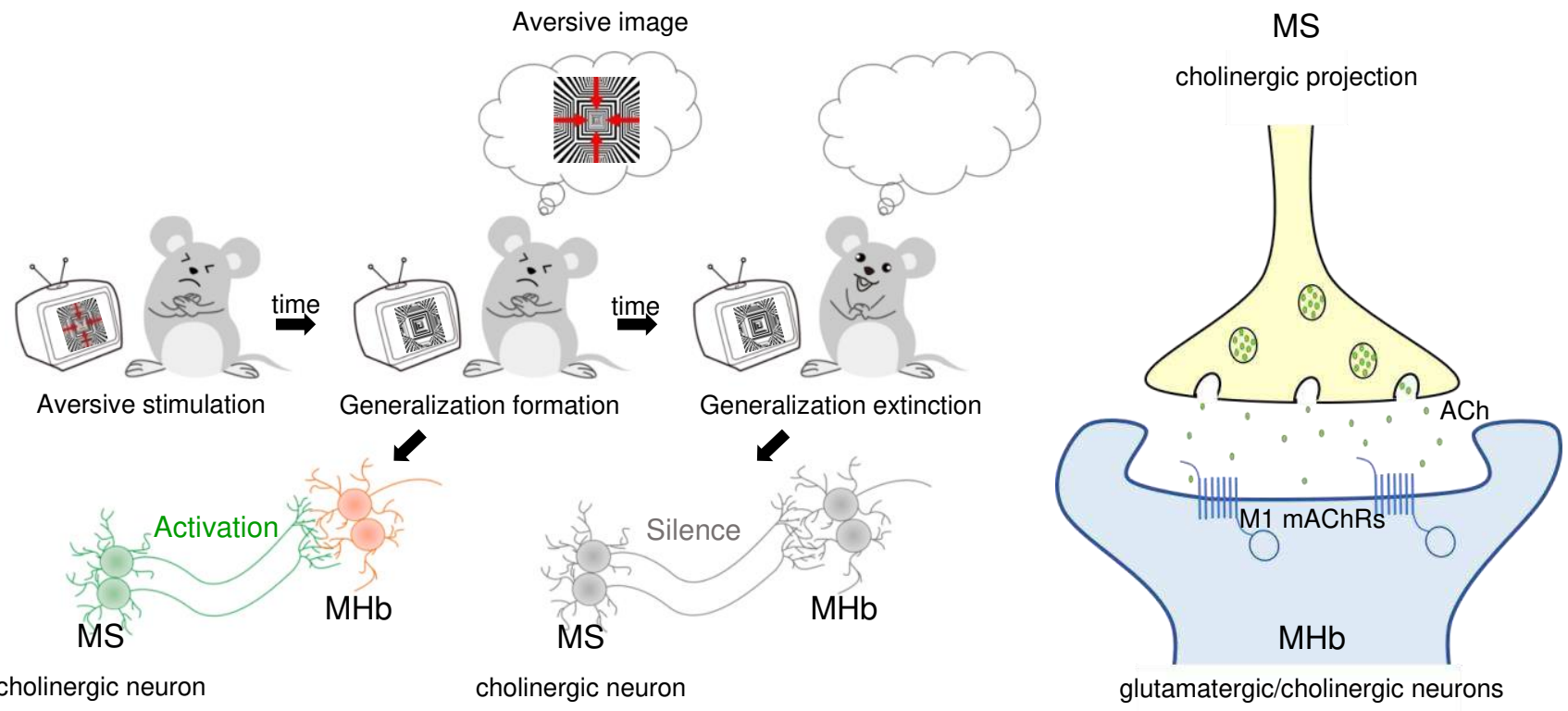


\section{Figures}

a

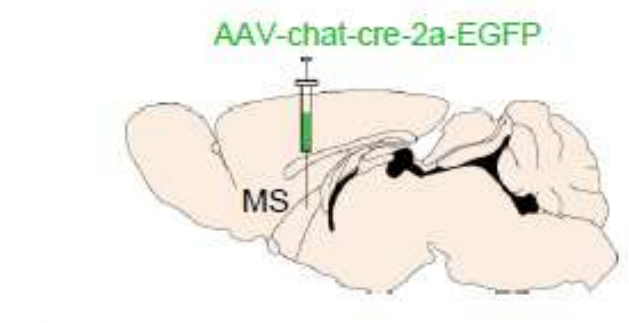

b

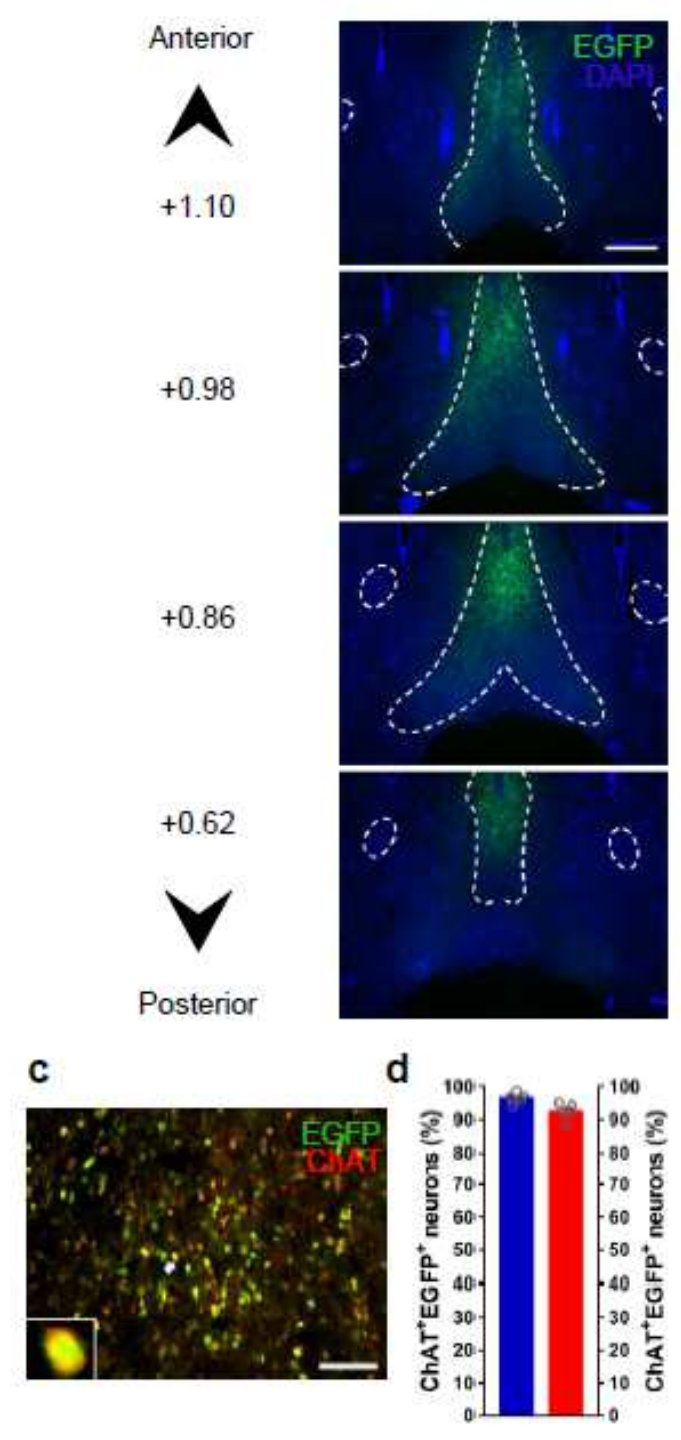

e

f
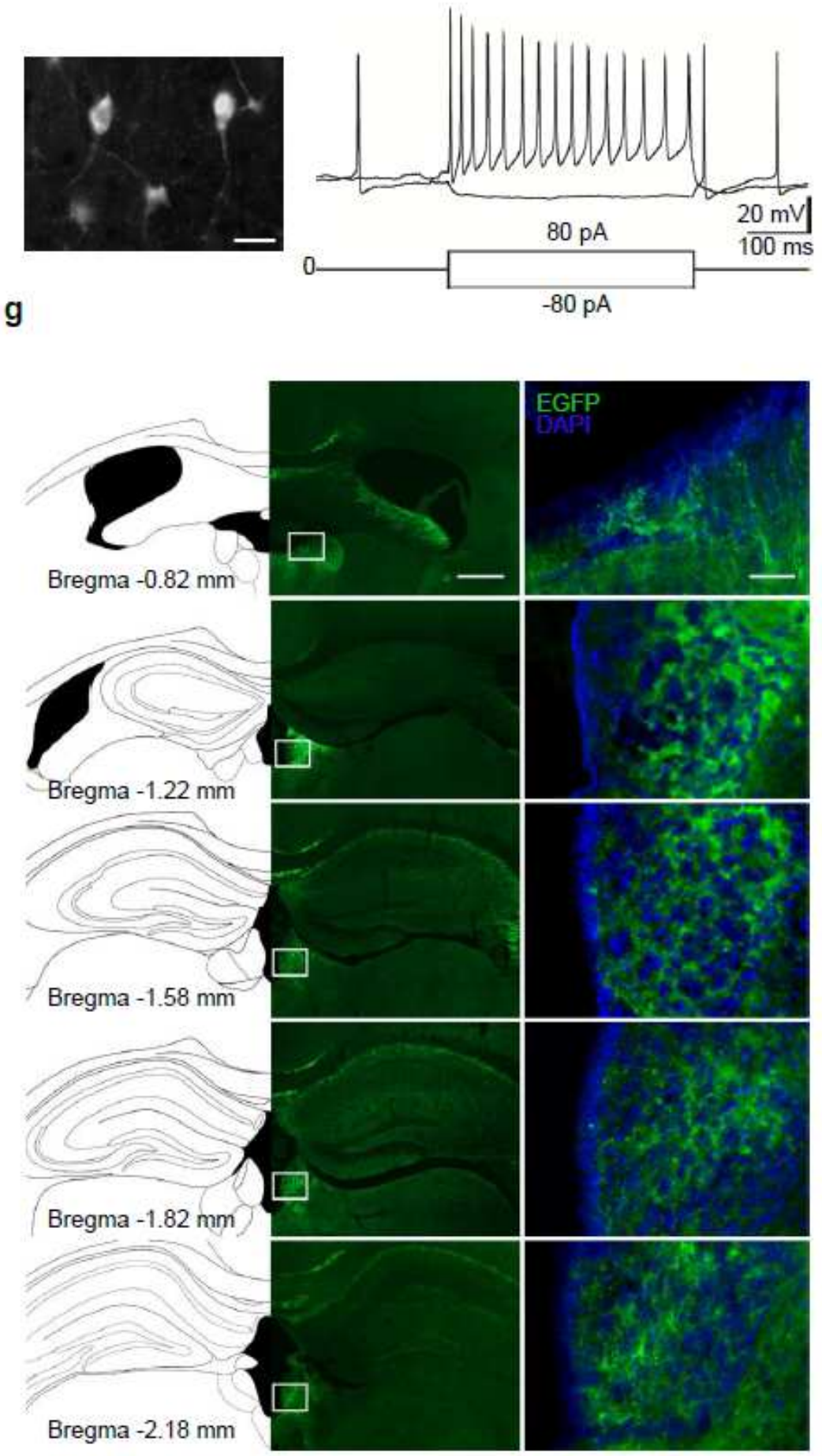

\section{Figure 1}

MS provides strong cholinergic projections to the MHb. a Schematic diagram of virus injection of AAVchat-cre-EGFP into the MS to track its cholinergic projections. b Representative images of AAV-chat-creEGFP expression in the MS three weeks after virus injection. The numbers correspond to anterior-posterior 
distance (in mm) from bregma. c Representative image of EGFP-labeled cholinergic neurons (green) stained with anti-ChAT (red) in the MS by immunohistochemistry. Inset, magnification of targeted neuron marked with a white arrow. d Quantification of the percentage of ChAT+ EGFP+ cells (blue) relative to all EGFP-labeled cholinergic neurons in the MS. Quantification of the percentage of ChAT+ EGFP+ cells (red) relative to all anti-ChAT neurons in the MS. $n=5$, four sections per mouse. e Representative image of EGFP-labeled MS cholinergic neurons in whole-cell patch-clamp recording after a 6-8 weeks expression of AAV-chat-cre-EGFP. $f$ Response of EGFP-labeled MS cholinergic neurons to intracellular current steps during whole-cell recording. Depolarizing current: +80 pA; hyperpolarization current: -80 pA. g Representative whole-brain mapping of EGFP-labeled cholinergic projections from the MS to the MHb. Scale bar, $400 \mu \mathrm{m}$ (b), $100 \mu \mathrm{m}$ (c), $50 \mu \mathrm{m}$ (e), $400 \mu \mathrm{m}$ (g, middle) and $50 \mu \mathrm{m}$ (g, right). Data are mean \pm s.e.m. Additional statistical information can be found in Supplementary Table 2.

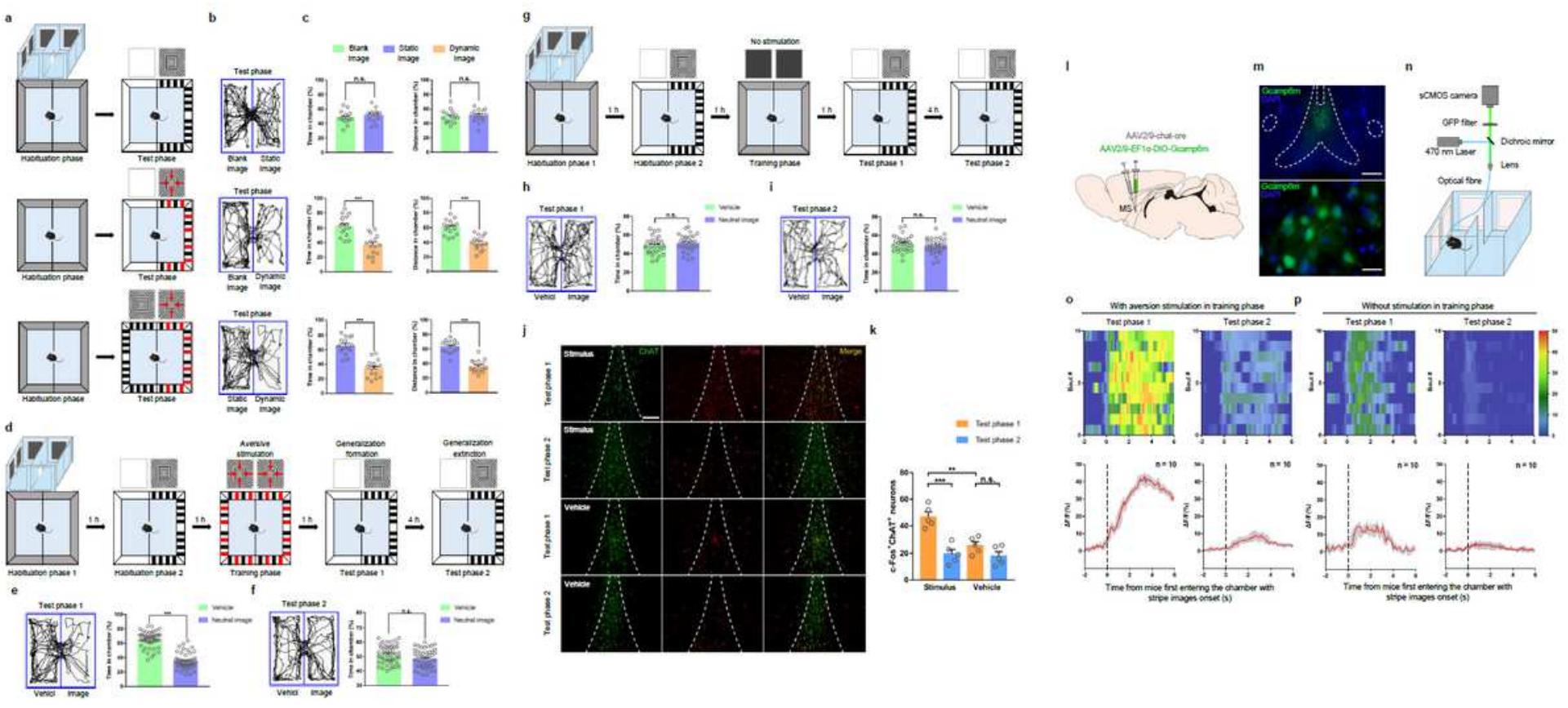

Figure 2

MS cholinergic neuron supports rapid generalization formation and extinction of visual aversion in mice. a Scheme of a visual aversion task based on real-time place preference (RTPP) caused by vision. Red arrows show movement direction of the dynamic stripe image. b Representative trace of the locomotion in visual aversion task in mice. c Quantification of the percentage of time spent (c, left) or distance moved (c, right) in each chamber during test phase. $n=15$. $d$ Scheme of a generalization task with visual aversion stimulus during training phase. e, $f$ Representative movement trace and quantification of the percentage of time spent in each chamber in test phase 1 and 2 (test phase 1: generalization formation phase of visual aversion, e; test phase 2: generalization extinction phase, $f$ ). $n=50$. g Scheme of a generalization task without visual aversion stimulus during training phase. $h$, i Representative movement trace and quantification of the percentage of time spent (by mice without suffering aversion stimulus) in each chamber in test phase 1 and 2. $n=30$. j Representative images of ChAT and c-Fos immunostaining in MS neurons of mice subjected to aversion generalization task. k Quantification of c-Fos+ ChAT+ 
neurons in the MS. $n=5$, four sections per mouse. I Schematic diagram of virus injections and optic fiber implantation for recording of GCaMP fluorescence signals of MS cholinergic neurons. $m$ Representative images of GCaMP6m expression in the MS. $n$ Schematic diagram of the fiber photometry setup. Ca2+ transients were recorded from GCaMP6m-expressing MS cholinergic neurons in mice subjected to visual aversion generalization task. o, p Upper panel: the heatmap illustration of $\mathrm{Ca} 2+$ signals aligned to the onset of aversion generalization formation or extinction assessment. Each row represents one bout. Lower panel: the peri-event plot of the average $\mathrm{Ca} 2+$ signal transients. $0 \mathrm{~s}$ means time from mice first entering the chamber with stripe images onset. $n=10$. Thick red lines indicate mean and shaded areas indicate SEM. Mice with aversion stimulus (o), mice without aversion stimulus (p). Scale bar, $200 \mu \mathrm{m}(\mathrm{j})$, $400 \mu \mathrm{m}\left(\mathrm{m}\right.$, top) and $50 \mu \mathrm{m}\left(\mathrm{m}\right.$, bottom). Data are mean \pm s.e.m. ${ }^{\star *} \mathrm{P}<0.01,{ }^{\star * *} \mathrm{P}<0.001$ (two-tailed unpaired Student's $t$ test for $c, e, f, h$ and i; one-way ANOVA with Tukey's post-hoc analysis for $k$ ). Exact $p$ values and additional statistical information can be found in Supplementary Table 2.
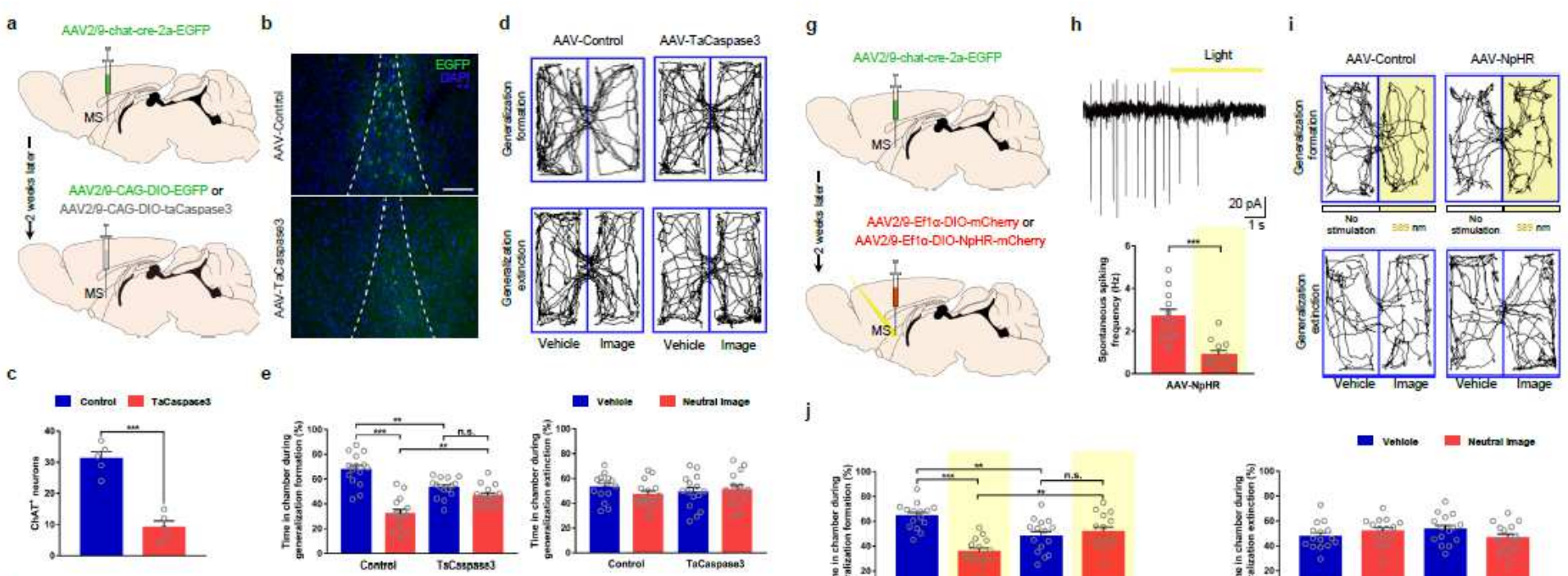

j
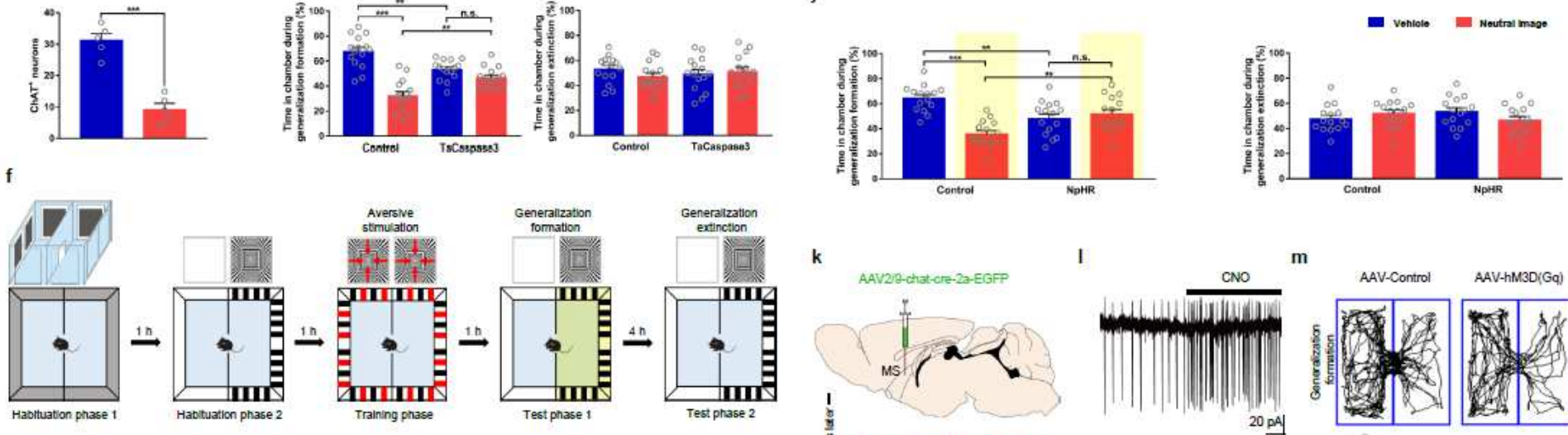

n
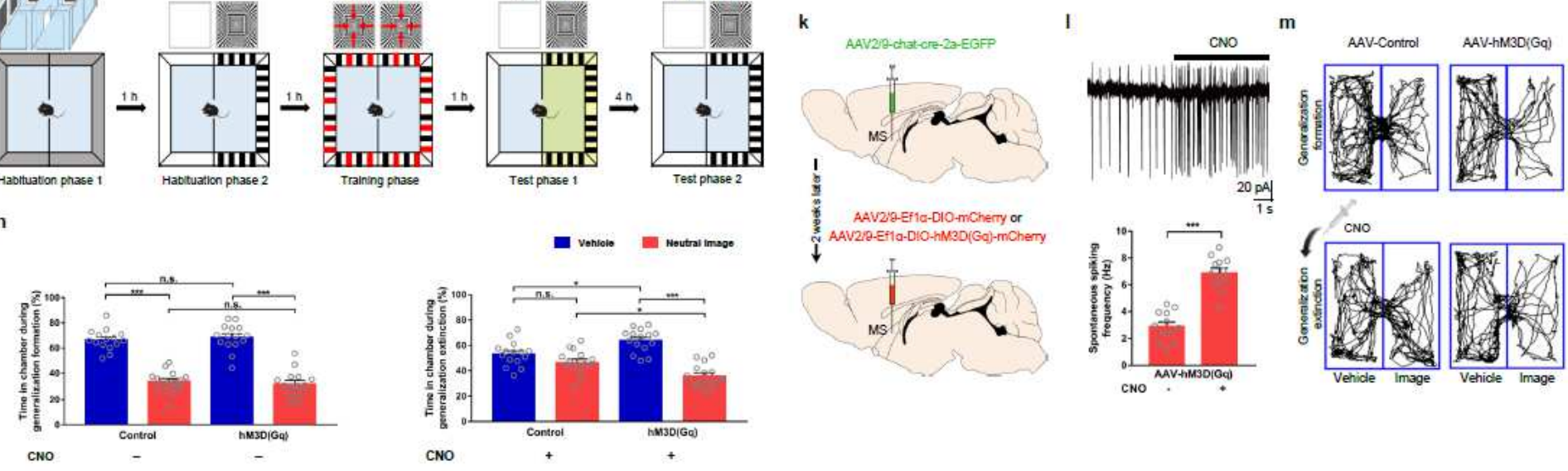

\section{Figure 3}

MS cholinergic neuron activity is necessary for generalization formation and extinction of visual aversion. a Schematic diagram depicting virus injections for apoptosis of MS cholinergic neurons. b, c Representative images (b) and quantification (c) of EGFP-labeled MS cholinergic neurons in taCaspase3- 
expressing or control mice. $n=5$, four sections per mouse. $d$, e Data of visual aversion generalization task in mice whose MS cholinergic neurons were inhibited with apoptosis manipulation (representative trace of mice, $d$; quantification of the percentage of time spent in each chamber during generalization formation and extinction phases, e). $n=15$. $f$ Scheme of visual aversion generalization task with optogenetic inhibition approach using a yellow-light (589 nm, $10 \mathrm{~mW}$ ). g Schematic diagram depicting virus injections for optogenetic inhibition of MS cholinergic neurons. h Yellow light decreased spontaneous firing in MS cholinergic neurons expressing NpHR-mCherry in brain slices. $n=12$ cells from four mice. $i, j$ Data of visual aversion generalization task in mice whose MS cholinergic neurons were inhibited with optogenetic manipulation (representative trace of mice, i; quantification of the percentage of time spent in each chamber during generalization phases, $\mathrm{j}$ ). $\mathrm{n}=15$. $\mathrm{k}$ Schematic diagram depicting virus injections for chemogenetic activation of MS cholinergic neurons. Bath application of CNO $(5 \mu \mathrm{M})$ increased spontaneous firing in MS cholinergic neurons expressing hM3Dq-mCherry in brain slices. $\mathrm{n}=$ 12 cells from four mice. $m, n$ Data of visual aversion generalization task in mice whose MS cholinergic neurons were activated with chemogenetic manipulation (representative trace of mice, m; quantification of the percentage of time spent in each chamber during generalization phases, $n$ ). Mice were intraperitoneally injected with CNO $(2.5 \mathrm{mg} / \mathrm{kg})$ after generalization formation test. $\mathrm{n}=15$. Scale bar, 200 $\mu \mathrm{m}$ (b). Data are mean \pm s.e.m. ${ }^{*} \mathrm{P}<0.05$, ${ }^{\star *} \mathrm{P}<0.01$, ${ }^{\star \star \star} \mathrm{P}<0.001$, n.s.: $\mathrm{p}>0.05$ (two-tailed unpaired Student's $t$ test for $c, h$ and l; one-way ANOVA with Tukey's post-hoc analysis for $e, j$ and $n$ ). Exact $p$ values and additional statistical information can be found in Supplementary Table 2. 
a

AAV2/Retro-Ef1a-DIO-mCherry or AAV2/Retro-Efta-DIO-hM4D(Gi)-mCherry

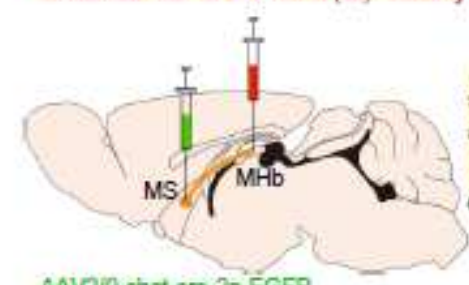

AAV2/2-chat-cre-2a-EGFP

b

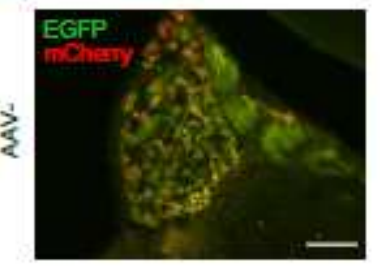

c

CNO

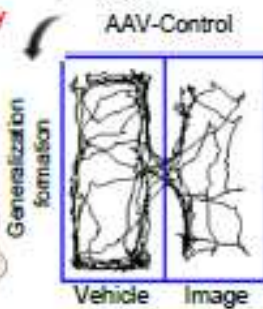

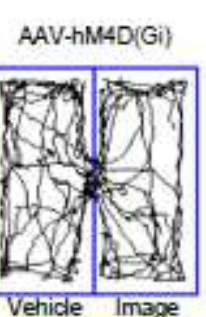

d

vehiole
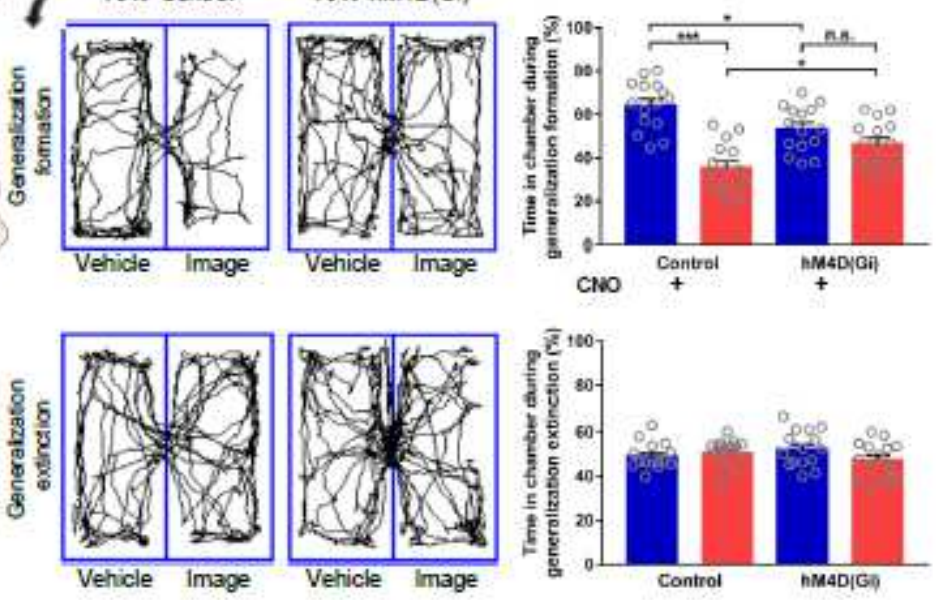

e
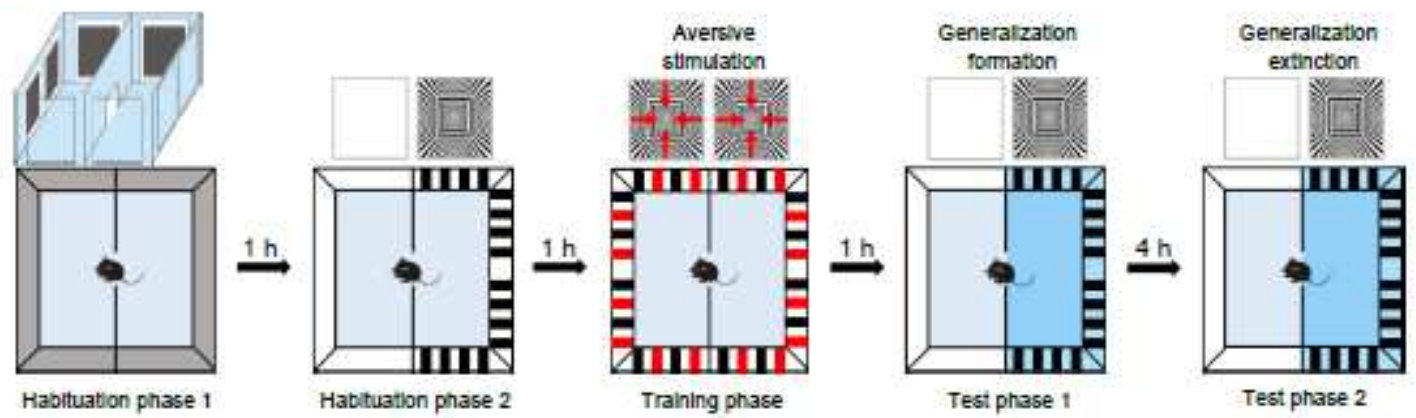

f

h

AAV2/Retro-EHId-DIO-mCherry or AAV2Retro-Ef10-DIO-ChR2-mCherry
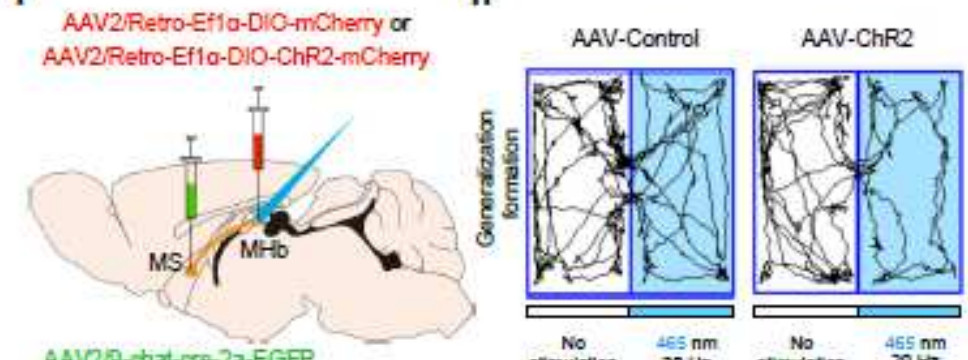

i
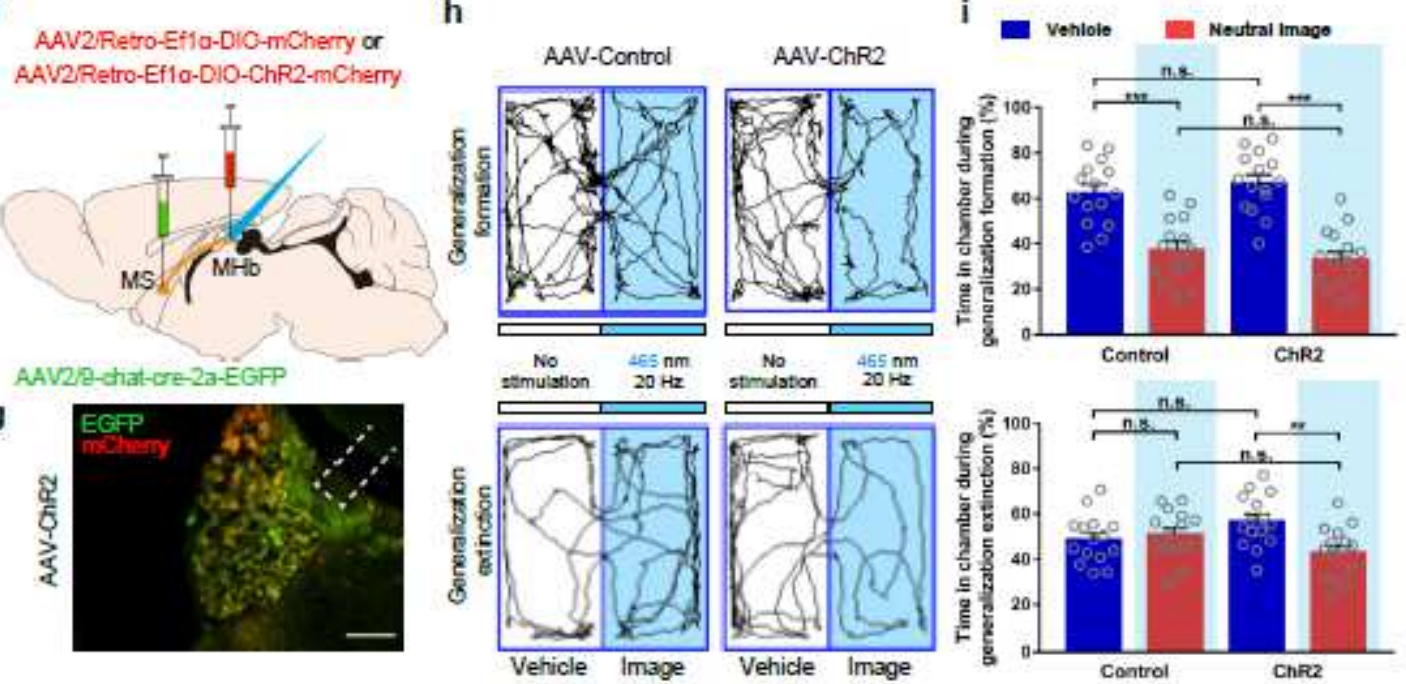

Figure 4

$\mathrm{MS} \triangle \mathrm{MHb}$ cholinergic circuit drives generalization formation and extinction of visual aversion. a Schematic diagram depicting virus injections for chemogenetic inhibition of MS $\triangle \mathrm{MHb}$ cholinergic circuits. b Representative image of AAVs expression in the MHb three weeks after AAV-hM4D(Gi) injection. c, d Data of visual aversion generalization task in mice whose MS $\triangle \mathrm{MHb}$ cholinergic circuits were inhibited with chemogenetic manipulation (representative trace of mice, c; quantification of the percentage of time spent in each chamber during generalization formation and extinction phases, d). Mice were 
intraperitoneally injected with CNO $(2.5 \mathrm{mg} / \mathrm{kg}) 30$ min before generalization formation test. $\mathrm{n}=15$. e Scheme of visual aversion generalization task with an optogenetic activation approach using blue-light pulse $(465 \mathrm{~nm}, 20 \mathrm{~Hz}, 40 \mathrm{~ms})$. f Schematic diagram depicting virus injections for optogenetic activation of $\mathrm{MS} \triangle \mathrm{MHb}$ cholinergic circuits. $g$ Representative image of AAVs expression in the MHb three weeks after AAV-ChR2 injection. $h$, i Data of visual aversion generalization task in mice whose MS $\square \mathrm{MHb}$ cholinergic circuits were activation with optogenetic manipulation (representative trace of mice, h; quantification of the percentage of time spent in each chamber during generalization formation and extinction phases, i). Scale bar, $100 \mu \mathrm{m}$ (b and g). Data are mean \pm s.e.m. ${ }^{*} P<0.05,{ }^{* \star} P<0.01$, ${ }^{\star \star *} P<0.001$, n.s.: $p>0.05$ (oneway ANOVA with Tukey's post-hoc analysis for $d$ and $i)$. Exact $p$ values and additional statistical information can be found in Supplementary Table 2. 
a

(

AAV2/Retro-Ef1a-DiO-mCherry (CNO)
or

AAV2/Retro-Ef1o-DIO-hM3D(Gq)-mCherry (CNO)

or

AAV2/Retro-Ef1a-DIO-hM3D(Gq)-mCherry (CNO+Pirenzepine)

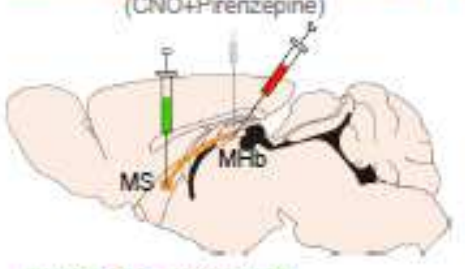

AAV2/e-chat-are-2aEGFP

b

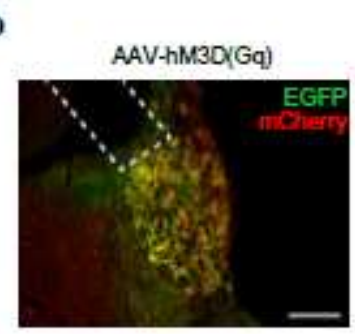

d
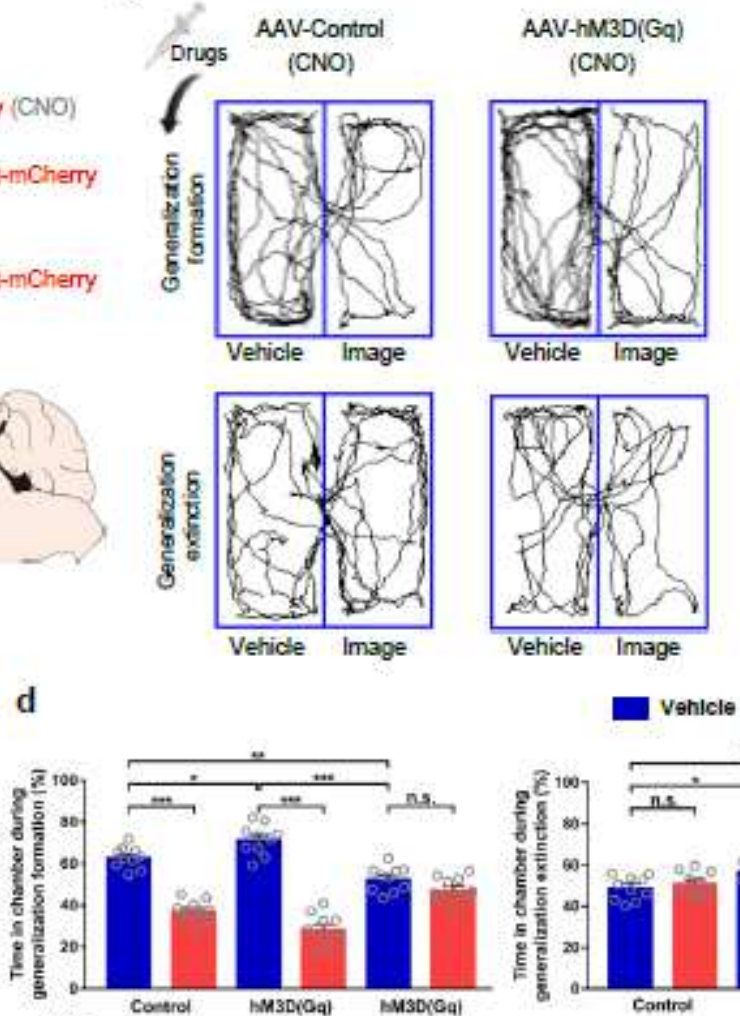

AAV-hM3D(Gq) (CNO+Pirenzepine)
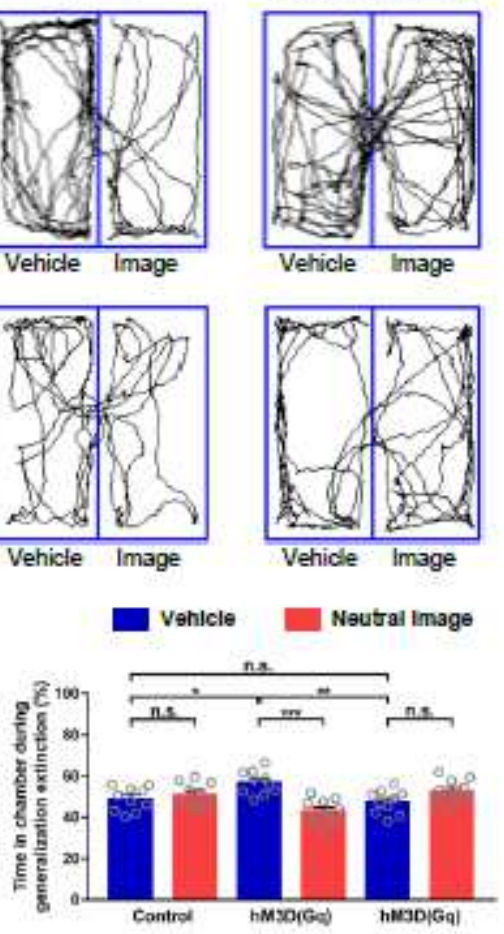

CNO

e
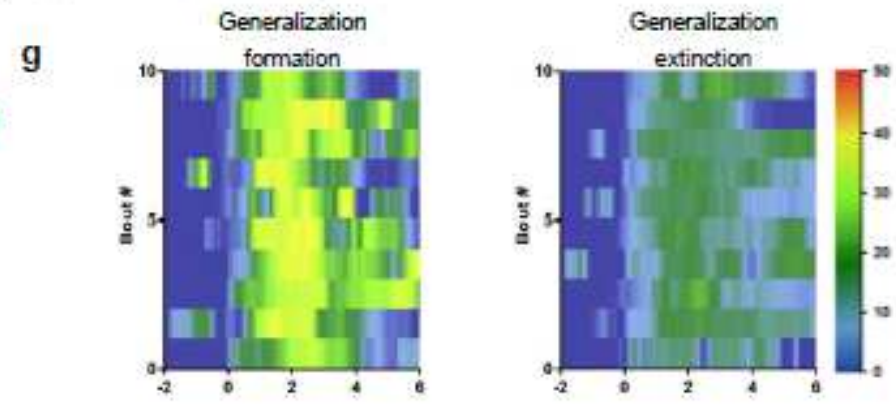

f

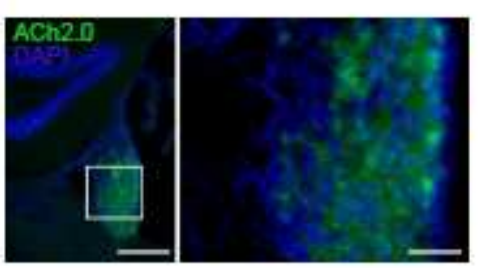

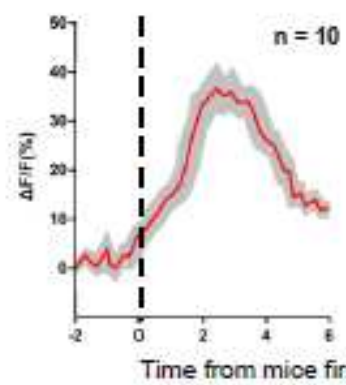

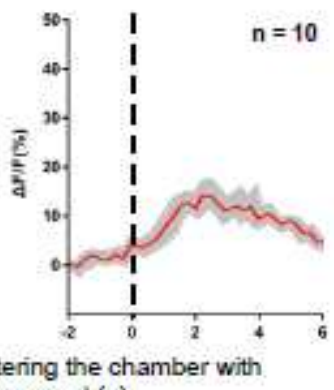

stripe images onset (s)

\section{Figure 5}

$\mathrm{M} 1 \mathrm{mAChRs}$ and glutamatergic neurons in the $\mathrm{MHb}$ are involved in generalization formation and extinction of visual aversion. a Schematic diagram depicting virus and drug injections for chemogenetic activation of MS $\triangle \mathrm{MHb}$ cholinergic circuits and blockade of MHb M1 mAChRs. b Representative image of AAVs expression in the $\mathrm{MHb}$ three weeks after $\mathrm{AAV}-\mathrm{hM} 3 \mathrm{D}(\mathrm{Gq})$ injection. c, $d$ Data of visual aversion generalization task in mice whose MS $\triangle \mathrm{MHb}$ cholinergic circuits were activated with chemogenetic manipulation while MHb M1 mAChRs were blocked by pirenzepine (representative trace of mice, c; 
quantification of the percentage of time spent in each chamber during generalization formation and extinction phases, d). Mice were injected with CNO $(3.0 \mu \mathrm{M} / 0.1 \mu \mathrm{L} /$ side $)$ or CNO + Pirenzepine $(1.5 \mu \mathrm{g} / 0.1$ $\mu \mathrm{L} /$ side) into the $\mathrm{MHb}$ by cannulas 30 min before generalization formation test. $\mathrm{n}=10$. e Schematic diagram of virus injections and optic fiber implantation for recording of acetylcholine fluorescence signals of MHb glutamatergic neurons. $f$ Representative images of ACh2.0 expression in the MHb three weeks after AAV-ACh2.0 injection. $g$ Acetylcholine transmissions in $\mathrm{MHb}$ glutamatergic neurons were involved in generalization formation ( $\mathrm{g}$, left) and extinction ( $\mathrm{g}$, right) of visual aversion. Upper panel, the heatmap illustration of acetylcholine signals aligned to the onset of individual generalization assessments. Each row represents one bout, and a total of 10 bouts are illustrated. Lower panel, the perievent plot of the average acetylcholine signal transients. $0 \mathrm{~s}$ means time from mice first entering the chamber with stripe images onset. $n=10$. Thick red lines indicate mean and shaded areas indicate SEM. Scale bar, $100 \mu \mathrm{m}$ (b), $200 \mu \mathrm{m}$ (f, left) and $50 \mu \mathrm{m}$ (f, right). Data are mean \pm s.e.m. ${ }^{*} P<0.05,{ }^{\star \star} P<0.01$, $\star \star \star P<0.001$, n.s.: $p>0.05$ (one-way ANOVA with Tukey's post-hoc analysis for $d$ ). Exact $p$ values and additional statistical information can be found in Supplementary Table 2. 

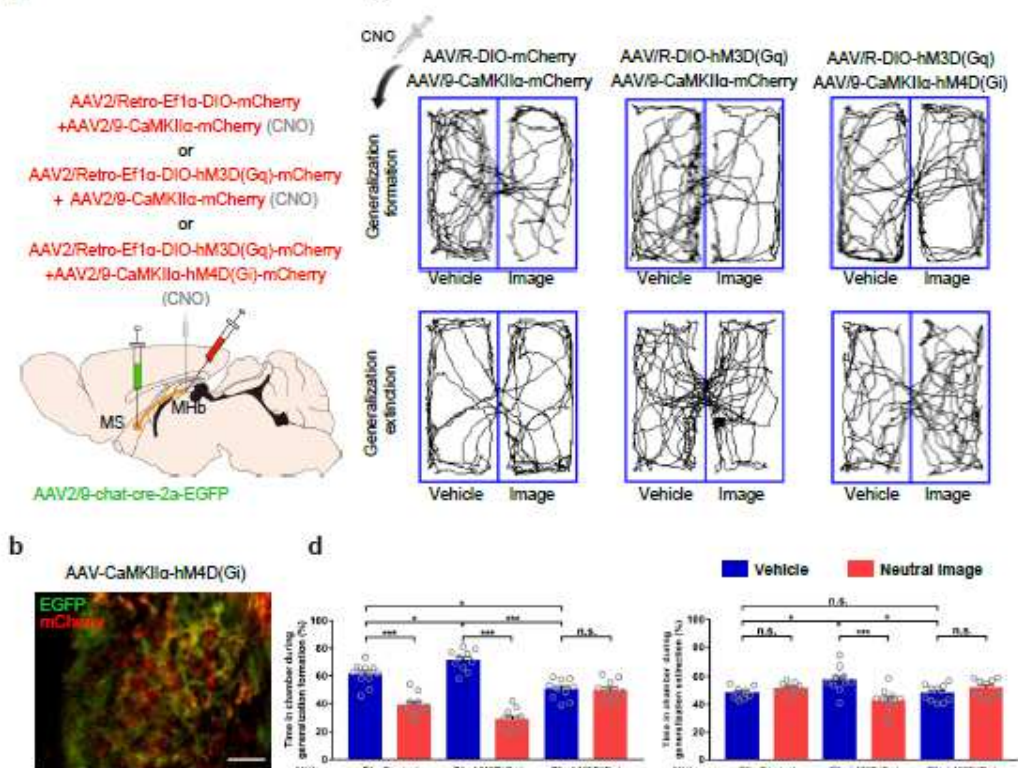

d
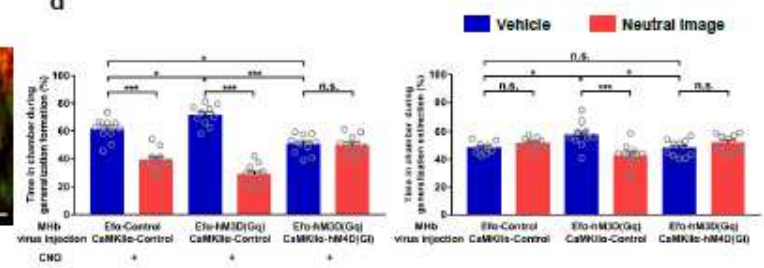

e

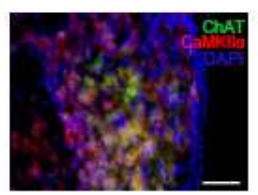

f

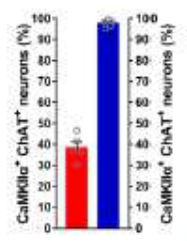

$$
\begin{aligned}
& \begin{array}{c}
\text { AAV2/-chat-cre } \\
+ \text { AAV2/-Efla-DIO-hM3D(Gq)-mCherry }
\end{array} \\
& \begin{array}{c}
\text { (ACSF) } \\
\text { or } \\
\text { AAV2o-chat-ore }
\end{array} \\
& \text { or }
\end{aligned}
$$

g AAV-hM3D(Gq) (CNO)
(CIO-hM3O(S)
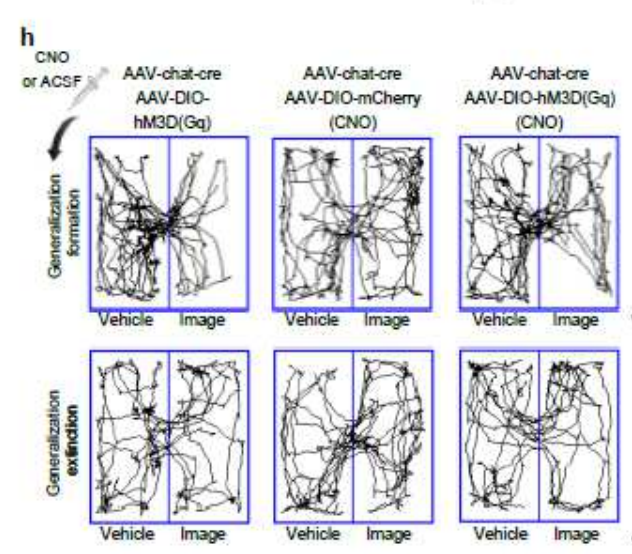

i
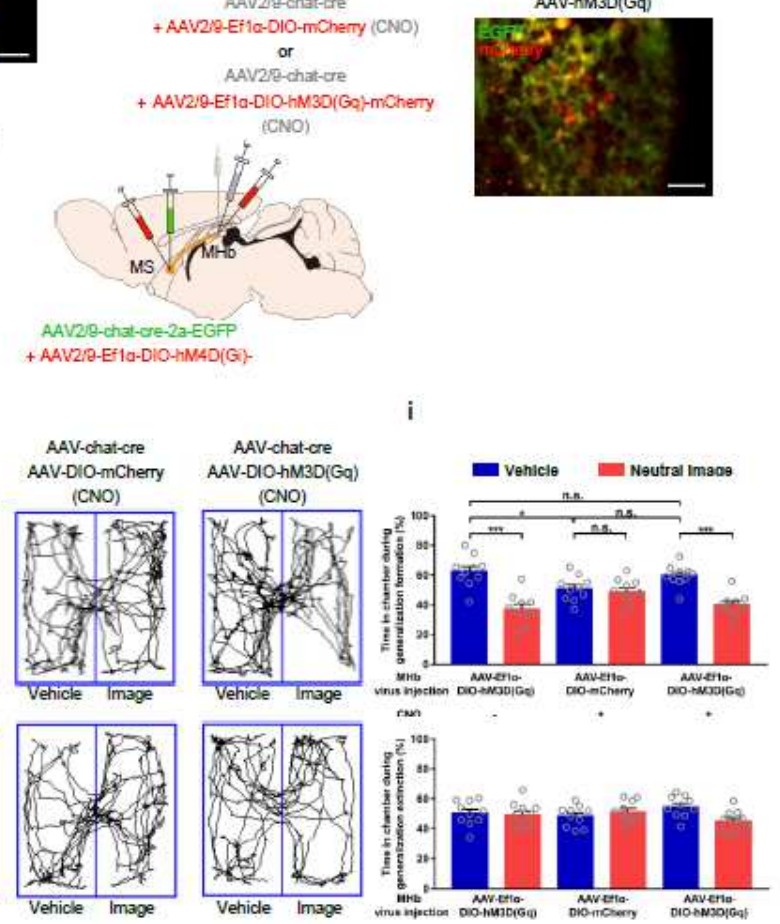

\section{Figure 6}

$\mathrm{MHb}$ glutamatergic/cholinergic neurons participate in generalization formation and extinction of visual aversion. a Schematic diagram depicting virus and drug injections for activation of MS $\otimes \mathrm{MHb}$ cholinergic circuits and inhibition of MHb glutamatergic neurons with chemogenetic approaches. b Representative image of expressions of AAVs (used in a) in the MHb. c, d Data of visual aversion generalization task in mice whose MS $₫ \mathrm{MHb}$ cholinergic circuits were activated while MHb glutamatergic neurons were inhibited 
with chemogenetic manipulations (representative trace of mice, c; quantification of the percentage of time spent in each chamber during generalization formation and extinction phases, d). CNO $(3.0 \mu \mathrm{M} / 0.1$ $\mu \mathrm{L} /$ side) was bilaterally injected into the $\mathrm{MHb}$ by cannulas 30 min before generalization formation test. $\mathrm{n}$ $=10$. e Representative image (e,top) and quantification (e, bottom) of co-localization of CaMKIla (red) with ChAT (green) in MHb neurons by immunohistochemistry. $n=5$, four sections per mouse. $f$ Schematic diagram depicting virus and drug injections for chemogenetic inhibition of MS $₫ \mathrm{MHb}$ cholinergic circuits and activation of $\mathrm{MHb}$ glutamatergic/cholinergic neurons with chemogenetic approaches. $\mathrm{g}$ Representative image of expressions of AAVs (used in $f$ ) in the MHb. h, i Data of visual aversion generalization task in mice whose MS $₫ \mathrm{MHb}$ cholinergic circuits were inhibited while $\mathrm{MHb}$ glutamatergic/cholinergic neurons were activated with chemogenetic manipulations (representative trace of mice, $\mathrm{h}$; quantification of the percentage of time spent in each chamber during generalization formation and extinction phases, i $)$. CNO $(3.0 \mu \mathrm{M} / 0.1 \mu \mathrm{L} /$ side) was bilaterally injected into the $\mathrm{MHb}$ by cannulas $30 \mathrm{~min}$ before generalization formation test. $\mathrm{n}=10$. Scale bar, $50 \mu \mathrm{m}$ (b, e and g). Data are mean \pm s.e.m. ${ }^{*} P<0.05$, ${ }^{\star * \star P}<0.001$, n.s.: $P>0.05$ (one-way ANOVA with Tukey's post-hoc analysis for $d$ and i). Exact $p$ values and additional statistical information can be found in Supplementary Table 2 .

a

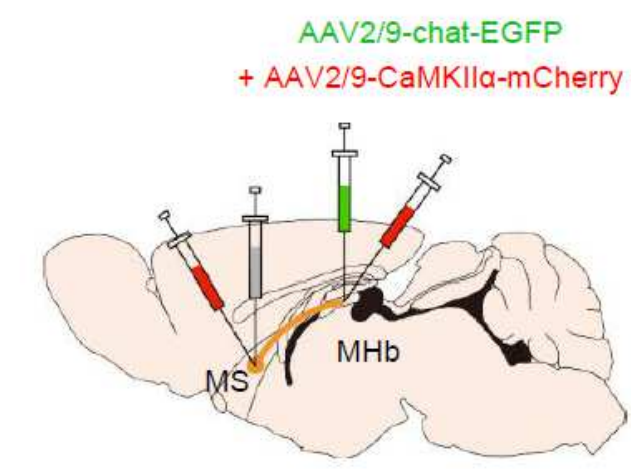

AAV2/9-chat-cre

+ AAV2/9-Ef1a-DIO-ChR2-mCherry

d

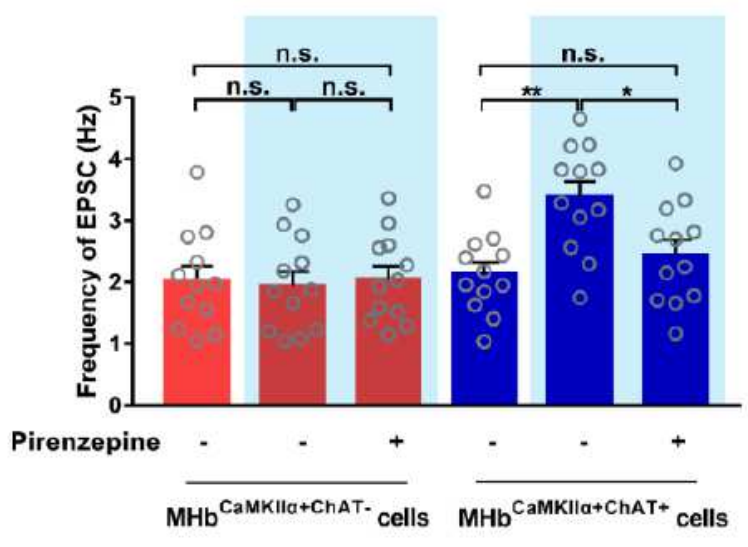

C

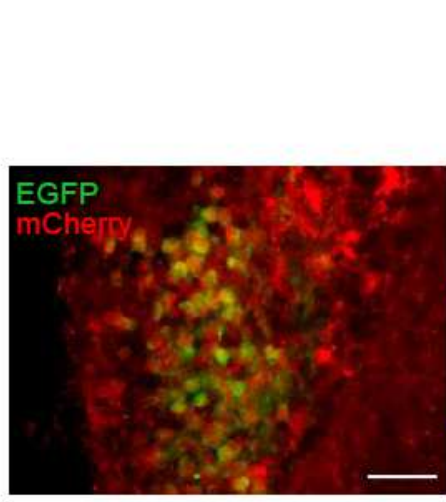

b

CaMKIIa+ChAT cell

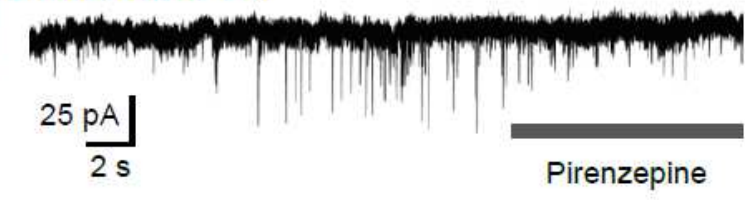

Figure 7

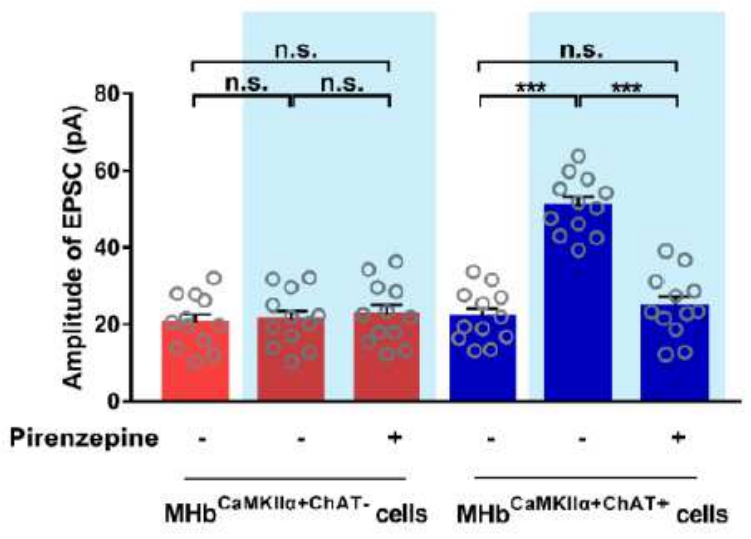


$\mathrm{MHb}$ glutamatergic/cholinergic neurons mediate regulatory effects of MS $\triangle \mathrm{MHb}$ cholinergic circuit via $\mathrm{M} 1$ mAChRs. a Schematic diagram depicting virus injections for whole-cell recording of EPSC in MHb neurons. b Representative image of AAVs expression in the MHb three weeks after AAV-ChR2 injection. $\mathrm{MHb}$ glutamatergic/cholinergic neurons (MHbCaMKIla+ChAT+) were labeled with chat-EGFP and CaMKIla-mCherry. MHb glutamatergic neurons without releasing acetylcholine (MHbCaMKIla+ChAT-) were labeled with CaMKIla-mCherry alone. Scale bar, $50 \mu \mathrm{m}$. c Representative traces of EPSCs in $\mathrm{MHbCaMKIla}+\mathrm{ChAT}+$ and MHbCaMKIla$+\mathrm{ChAT}$ - neurons. EPSCs were recorded at $-70 \mathrm{mV}$. Optogenetic stimulations $(465 \mathrm{~nm}, 5 \mathrm{~ms}, 20 \mathrm{~Hz})$ of ChR2+ axonal terminals and M1 mAChRs blockade were performed during the recording of EPSCs. $d$, e Quantification of frequency $(m)$ and amplitude $(n)$ of EPSCs. $n=12$ cells from four mice. Data are mean \pm s.e.m. ${ }^{*} P<0.05,{ }^{\star \star} P<0.01,{ }^{\star \star \star *} P<0.001$, n.s.: $P>$ 0.05 (one-way ANOVA with Tukey's post-hoc analysis for $m$ and $n$ ). Exact $p$ values and additional statistical information can be found in Supplementary Table 2.

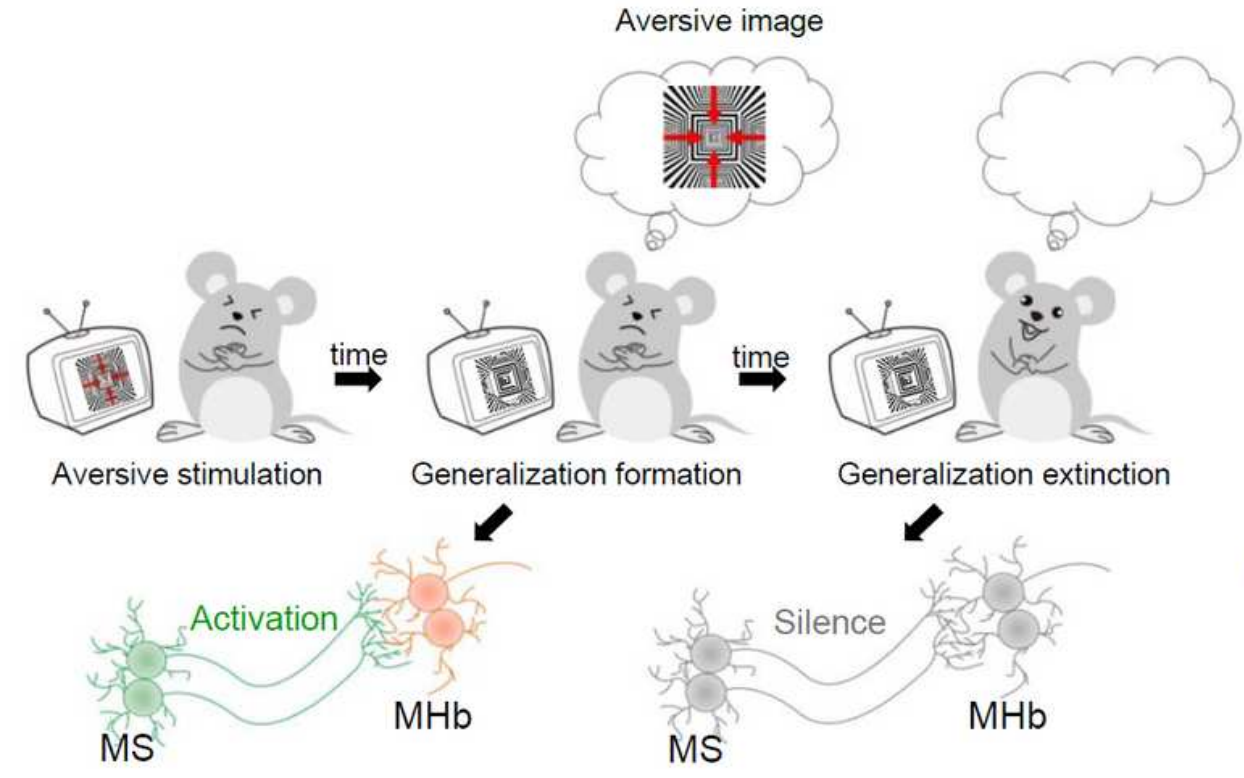

cholinergic neuron

cholinergic neuron

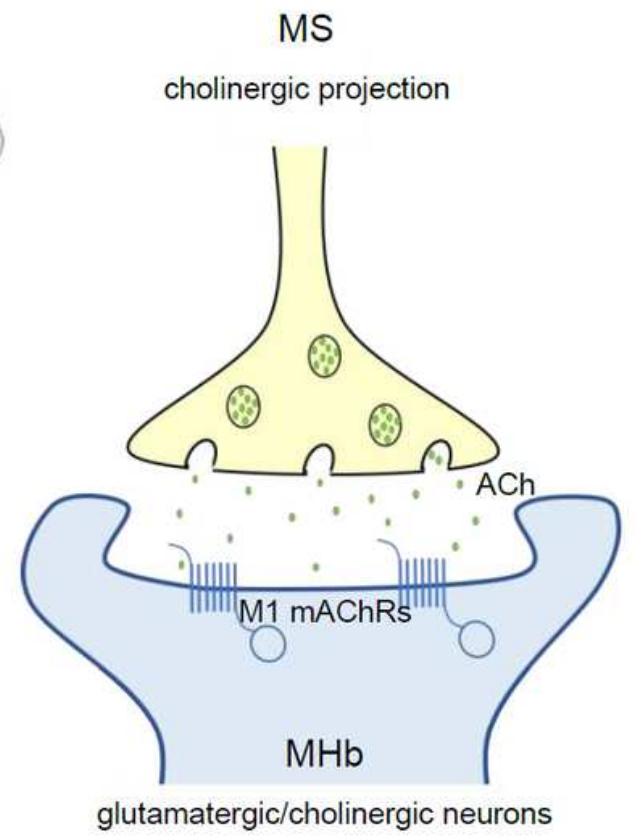

\section{Figure 8}

Conceptual diagrams for generalization formation and extinction of visual aversion mediated by $\mathrm{MS} \otimes \mathrm{MHb}$ cholinergic circuits. A model for visual aversion generalization formation and extinction is built whereby highly similarity between dynamic stripe image (aversive image) and static stripe image (neutral image). Activation of the MS $\triangle \mathrm{MHb}$ cholinergic circuit drives generalization formation while its silence supports subsequent extinction. Moreover, $\mathrm{MS} \triangle \mathrm{MHb}$ cholinergic projections modulate generalization formation and extinction of visual aversion via M1 mAChRs on downstream glutamatergic/cholinergic neurons.

\section{Supplementary Files}


This is a list of supplementary files associated with this preprint. Click to download.

- SupplementaryTable1.xlsx

- SupplementaryTable2.xlsx

- Supplementaryinformation.pdf 